\title{
XXVIIIth Meeting of the Canadian Congress of Neurological Sciences
}

\author{
Abstracts of the Scientific Program
}

\section{PLATFORM SESSIONS}

Thursday, June 17
A. Neurology
General Neurology
$1-6$
Multiple Sclerosis

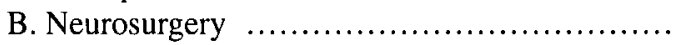
C. Pediatric Neurology
$12-22$
23-33
D. Neurology
Neuromuscular
34-39
Neuro-ophthamology
40-44

Friday, June 18

E. Neurology

F. Neurosurgical Oncolog

G. Clinical Neurophysiology ........................

H. Cerebral Ischemia

$45-55$

$56-66$

$67-77$

$78-88$

\section{PRIZE ABSTRACTS}

1. K.G. McKenzie Memorial Prize

Page S77

\section{Platform Presentations}

\section{A. NEUROLOGY}

1.

Structural and Functional Lesion Correlates of Hemispatial Neglect Using CT and SPECT Imaging

S.E. BLACK, L. EHRLICH, C. CALDWELL, J. P. SZALAI, S. STAPLETON and F. LEIBOVITCH (Toronto, Ontario)

Hemispatial neglect is hypothesized to result from damage to a distributed neural network for attention which involves the inferior parietal, dorsolateral frontal, and cingulate cortices and the thalamic reticular pathways. According to this model, damage in one major node of the network could deactivate other nodes, and the more regions deactivated, the more severe the neglect. Since functional disconnections can be assessed using SPECT imaging, we utilized this technieque to assess brainbehavior correlations in neglect. One hundred and forty-five consecutive stroke patients with CT confirmed single lesions were tested acutely on a standard neglect battery. CT and HMPAO SPECT scanning were performed within the first week and lesions were localized by a stereotactic method. In the 76

\section{POSTER SESSIONS}

Thursday, June 17

I. 1. Pediatric Neurology

2. Neuro-Oncology

3. Epilepsy

4. Neuromuscular

5. Headache

P1-P35

P36-P56

P57-P71

P72-P81

P82-P86

Friday, June 18

II. 1. Neurology

P87-P108

2. Clinical Neurophysiology .................... P109-P114

3. Neurosurgery …......................... P115-P126

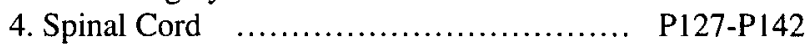

5. Cerebrovascular ............................. P143-P172 patients with right hemisphere damage so far analyzed, frontal, parietal and thalamic hypoperfusion on SPECT were significantly more frequent in neglecting patients $(p<.01)$. On logistic regression involving 7 cortical and subcortical regions frontal and parietal damage were the only predictors of neglect $(p<$ .001 ). In 26 patients with subcortical lesions on $C T$, the 12 with neglect all had frontal and parietal hypoperfusion on SPECT despite sparing of these regions on CT. Our findings support the concept that the frontal, parietal and thalamic areas are major nodes in the neural network for attention which is disrupted in the clinical syndrome of neglect.

2.

Fetal Dopaminergic Neural Transplantation for Treatment of Parkinson's Disease: Outcome After One Year

A. FINE, P. GAUDET, D. KING, R. HOLNESS, J. FISK, L. HEFFERNAN, R. POIRIER, R. CHALMERS, C. LEOPOLD, M. MACKAY-LYONS, A. LANG, D. CALNE and B. SNOW (Halifax, Nova Scotia).

We have performed experimental neural transplantation therapy for Parkinson's disease on 5 patients during the past year. Performance on a wide range of motor tasks was monitored 
throughout the day during at least six months preoperatively and throughout the postoperative period. At the time of transplantation, ventral mesencephalic tissue containing precursors of the dopaminergic substantai nigra was collected from fragments of 5 - 8 week gestational age fetuses, and stored under refrigeration. After virological and bacteriological testing, a cell suspension of uncontaminated tissue was prepared, and injected stereotaxically at multiple sites in the putamen contralateral to the most affected side. Patients received cyclosporin for the first six postoperative months. One year follow-up of the first two patients, including fluorodopa PET imaging, will be presented.

3.

A Therapeutic Trial of Glutamate Inhibition in Amyotrophic Lateral Sclerosis Using Lamotrigine

\section{A.A. EISEN, H. STEWART and D. CAMERON (Vancouver, British Columbia)}

Glutamate excitotoxicity is implicated in the pathogenesis of amyotrophic lateral sclerosis (ALS). We report the results of a double blind, placebo controlled, trial using $100 \mathrm{mg}$ of oral daily Lamotrigine (3,5-diamino-6(2,3 dichloropheny)-1,2,4-triazine) which inhibits glutamate release. At trial termination (mean duration $17.5 \pm 6.1$ months) 67 patients had been entered. 14 had withdrawn ( 7 active and 7 placebo) and 10 had died ( 6 active and 4 placebo). Mean age at entry was 58.8 years for both active and placebo groups with male:female ratios of 2.1:1 (active) and 0.6:1 (placebo). Neurological deficit was scored from 1 (best) - 16 (worst) based upon age of onset, bulbar and respiratory involvement, ambulation and functional disability. The mean change in scores for the active versus placebo groups over the trial period was $7.1 \pm 3.3$ and $9.0 \pm 3.3$ respectively. Changes in cortical magnetic stimulation studies carried out at 3 monthly intervals did not differ between the two groups. We conclude that Lamotrigine in the doses administered does not alter the course of ALS.

\section{4.}

Cerebrovascular Permeability Alterations in Acute Hypertension: Protective Effect of Colchicine

\section{S. NAG (Toronto, Ontario)}

The role of endothelial microtubules in cerebrovascular permeability alterations was determined by studying the effect of pretreatment with colchicine on cerebrovascular permeability alterations in rats with norepinephrine-induced acute hypertension. Horseradish peroxidase (HRP) was used as a marker of permeability alterations. Wistar-Furth rats were injected with HRP ( $250 \mathrm{mg} / \mathrm{kg})$ i.v. followed by a 2 minute i.v. infusion of norepinephrine $(1.5 \mathrm{ug} / \mathrm{ml} / \mathrm{min})$. Rats were then perfused with fixative, their brains sliced and reacted for the demonstration of HRP reaction product. Cerebral findings in acutely hypertensive rats were similar to our previous observations. Hypertensive rats developed increased cerebrovascular permeability to HRP which was in the form of HRP leakage confined to vessel walls or as multifocal areas of HRP extravasation in the cerebral cortex and white matter in $75 \%$ of brain slices examined. Rats pretreated with colchicine ( $2 \mathrm{mg} / \mathrm{kg}$ ) i.p., 4 and 16 hours prior to the hypertensive episode showed marked reduction in cerebrovascular permeability alterations to HRP and only few vessels showed increased permeability to HRP. These results indicate that endothelial microtubular dysfunction contributes to increased cerebrovascular permeability to protein in pathological states since permeability is reduced in the presence of a microtubule-stabilizing agent.

Supported by: the Heart \& Stroke Foundation of Ontario.

\section{5.}

\section{Cerebral Perfusion Index: A New Method of Assessing} Stroke Outcome

A.V. ALEXANDROV, L.E. EHRLICH, C.F. BLADIN and J.W. NORRIS (Toronto, Ontario)

We devised a non-invasive method of evaluating cerebral perfusion, the cerebral perfusion Index (CPI) based on fast qualitative evaluation of TCD and SPECT results in the acute stage of cerebral ischemia. It is calculated by multiplying the values of one of four TCD-patterns (normal, collateral, stenotic, and occlusive) by one of five SPECT-patterns (normal, high, mixed, low and severe perfusion deficit). In 20 consecutive cases of cerebral ischemia we performed TCD and SPECT within the first 5 hours after onset, calculated CPI and compared it prospectively to short-term recovery scored by the Canadian Neurological Scale (CNS). As a clinical predictor the Index was better than the inital CNS scoring done at the time of admission. CPI was valuable in cases of strokes in evolution, and in early differentiation of reversible symptoms (TIA's). In 5 cases of recurrent strokes CPI changed dramatically, followed by clinical deterioration.

These preliminary data suggest that CPI has a potential advantage in predicting outcome over clinical examination of stroke patients, and may also differentiate underlying pathogenesis.

6.

A New Method of Measuring Carotid Stenosis - the Toronto Stenosis Index

\section{C.F. BLADIN, A.V. ALEXANDROV and J.W. NORRIS (Toronto, Ontario)}

Methods of measuring ICA stenosis are primarily those used in the NASCET, ECST trials. Limitations with these techniques include ICA post-stenotic narrowing or dilatation, extensive atheroma, and difficulties with carotid bulb estimation. We developed a new technique, the Toronto Stenosis Index (TSI), that avoids problems of distal ICA or carotid bulb measurements. The TSI is based on the known fixed anatomical relationship between CCA diameter, and that of the ICA $(1.19 \times$ 
CCA diam = ICA bulb diam). ICA diameter can thus be estimated from measurement of the CCA. We evaluated 32 NASCET patients with stenosis measured by "eyeball", NASCET, TSI, and Doppler methods. There was no significant correlation between "eyeball", NASCET and Doppler, but there was between TSI and Doppler $(r=0.93)$. The poorest correlation was between NASCET and Doppler. Ten consecutive patients undergoing carotid endarterectomy were assessed using NASCET, TSI, Doppler, and finally plaque planimetry itself. Only TSI and Doppler showed any correlation with pathology. We conclude that Doppler and TSI represent the best techniques for accurate measurement of carotid stenosis. The NASCET method significantly underestimates the degree of stenosis, excluding some patients with threatened stroke from carotid endarterectomy.

\section{7.}

Improvement of Muscular Strength by Maprotiline (MAP) in Multiple Sclerosis

E. POURCHER, R.H. BOUCHARD, P. BARUCH, J.-P. BOUCHARD, D. LAFOND and C. RICHARDS (Quebec, Quebec)

Physical fatigue is common in multiple sclerosis (MS) and is often perceived as the most disabling symptom between relapses. Its relationship to inflammatory disease activity, neurological disability and depression (which is, like fatigue, more common and more severe in MS) is still poorly understood. Twelve patients with a clinically definite diagnosis of MS ( 2 males, 10 females: mean age $37.8 \pm 8.5$ ) were chosen for chronic fatigue symptomatology defined as a sense of physical tiredness and lack of muscular resource during an effortful task. They received a fixed schedule of lorazepam $0.5 \mathrm{mg} 1 / 2$ bid for two weeks; then, in a single-blind protcol: lorazepam + placebo (4 weeks); then lorazepam + MAP $25 \mathrm{mg}$ tid (4 weeks). They were assessed monthly by a psychiatrist using Montgomery-Asberg Depression RS, by a neurologist using the Kurtzke Expanded Disability index, and by a physiotherapist who measured the patients maximal static strength (MSS) and static endurance (SE) with Cybex 11 isokinetic system. Results: Friedman's twoway ANOVA revealed a significant difference between repeated measures of MSS $(P=0.017)$ but not of SE index. Parametric two-way ANOVA followed by post-hoc comparisons revealed a significant difference between the evaluation of MSS at the end of MAP treatment versus initial MSS measure $(p<0.01)$ and versus MSS measure after placebo $(p<0.05)$. However, the between-groups main effect for the existence of depression was not significant. Thus, the beneficial effect of MAP on muscular strength seems independant of its antidepressant effect.
8.

\section{A Possible Long-Term Protective Effect of Pregnancy on the Course of Multiple Sclerosis}

D.L. LUSSIER, C. MASSE, J. PLEINES, J. BLAMBERT and P. DUQUETTE (Montreal, Quebec)

Objective: Determining the long-term effect of pregnancy on the course of MS.

Background: Recent studies have demonstrated that pregnancy does not aggravate the immediate course of multiple sclerosis (MS), despite a mild increase in activity in the early postpartum period. The long-term effect of pregnancy on the course of MS is less well documented.

Design: A cohort of 26 women with MS cumulating 36 pregnancies was followed prospectively (average follow-up: •• years), and compared to women without pregnancies, but similar disease characteristics (age of onset, disease type and severity). We compared the exacerbation rates and a life-table analysis with a gradual end-point (using the EDSS level) was construed.

Results: We have observed a decline in the exacerbation rate during the pregnancy period and a slight increase in the first post-partum trimester, confirming previous studies. The exacerbation rate decreased over time in the 2 groups (reflecting the natural course of MS). although not reaching statistical significance, the invalidity in the pregnancy cohort tended to be delayed during the long-term follow-up.

Conclusion: Pregnancy could exert a long-term protective effect on the course of MS.

9.

Proton Magnetic Resonance Spectroscopic Imaging of Periventricular Plaque in Multiple Sclerosis Patients: Comparison with Single Voxel Acquisitions

E.P.J. PIORO, G.S. FRANCIS, J.P. ANTEL, L. COLLINS and D.L. ARNOLD (Montreal, Quebec)

Previous studies from our laboratory on patients with multiple sclerosis (MS) have shown that single voxel (SV) proton magnetic resonance spectroscopy (MRS) is a useful, noninvasive technique for characterization of metabolic changes within evolving (increased choline/creatine ratios acutely) and chronic (decreased $\mathrm{N}$-acetylaspartate/creatine ratios) periventricular demyelinating lesions. However, restrictions in size, shape and positiong of the volume of interest in SV MRS preclude detailed evaluations of the spatial distribution of metabolic abnormalities within or surrounding the plaque. We have examined 30 patients with relapsing/remitting and chronic progressive MS using proton MR spectroscopic imaging (MRSI) which provides a map of the regional distribution of metabolites in the brain. During the same examination, we also obtained SV MRS centered on the corpus callosum for comparion. Both techniques showed a decrease in the relative resonance intensity of $\mathrm{N}$-acetylaspartate. However, MRSI allowed more accurate and tailored evaluation of the metabolic lesion volume. MRSI appears to have advantages over SV MRS for analysis of MS pathology. 
10.

\section{Computer Assisted Determination of Multiple Sclerosis} Lesions in Magnetic Resonance Images

\section{S.J. KARLIK, J.R. MITCHELL, D.H. LEE and A. FENSTER (London, Ontario)}

Although MRI is the technique of choice for the diagnosis of MS, quantifying the number of lesions and assessing their changes between exams can be an arduous and time-consuming procedure. Our computerized system is a graphical tool which determines a 2-D histogram given a pair of input images. The user adjusts interactively the size and placement of regions of interest to reveal structures in the input images with similar proton density (PD) and T2 values. The histogram regions are passed to a second level which classifies the volume into white matter, grey matter, CSF, and MS lesion. The third level is composed of two modules used to render the segmented structures and to analyze the volumes of the segmented tissues. 2-D histograms reveal three distinct tissue clusters in controls. A distinct peak extending from the white matter/grey matter cluster which exhibited increased PD and T2 was only observable in slices with evident MS lesions. Many true MS lesion voxels have PD and T2-weighed intensities which overlap that of normal white and grey matter. Our system can be used to visualize and monitor the changes in time many MS lesions in the 3-D volume of the brain.

11.

Relative Frequency of Anti-MBP and Anti-PLP in Cerebrospinal Fluid of Optic Neuritis and Multiple Sclerosis Patients

\section{K.G. WARREN and I .CATZ (Edmonton, Alberta)}

Myelin basic protein (MBP) and proteolipid protein (PLP) were purified from normal human brain tissue. Autoantibodies to MBP (anti-MBP) and PLP (anti-PLP) were detected in cerebrospinal fluid (CSF) of patients with optic neuritis and multiple sclerosis (MS) by a solid phase radioimmunoassay.

Anti-MBP was elevated in $47 / 53$ optic neuritis patients. Anti-PLP was detected in the remaining 6 patients which did not have measurable levels of anti-MBP. Antibodies to both antigens were not found in any of the 53 optic neuritis CSFs.

369 clinically definite multiple sclerosis patients were clinically divided into 173 with acute relapses, 109 with progressing disease and 87 in remission.

-169/173 acute relapses had increased anti-MBP, 3/173 had

increased anti-PLP and $1 / 173$ had no antibody.

-108/109 progressings had increased anti-MBP and 1/109

had increased anti-PLP.

-15/87 remissions had somewhat elevated anti-MBP, none had anti-PLP.

From an immunochemistry point of view there may be at least two forms of MS: a common form highly associated with elevated anti-MBP and a more infrequent form associated with detectable anti-PLP. To date, autoantibodies to these two myelin proteins were not simultaneously detected in any CSF of individual optic neuritis or multiple sclerosis patient.

\section{B. NEUROSURGERY}

12.

A National Program for the Comprehensive Care for the Brain Injured - The Neurosurgeon's Role

N.C. HILL (Winnipeg, Manitoba)

Following consultation at local, provincial and national levels, a comprehensive program for the care of the brain injured, primarily due to trauma, has been completed and instituted in Manitoba covering a catchment area of 1.2 million. The program has resulted in improved patient care with corollary benefits of improved efficiency in the use of neurosurgical resources especially hospital beds and cost savings now estimated in excess of 4 million dollars in hospital costs alone. The final design of this program involved the cooperation of a neurosurgeon, a physical medical specialist, a neuropsychologist, a representative of the private sector (in this case, the Director of the Head Injury Association) and representatives of the Government sector (two senior officials of provincial social agencies). Statistics indicate that $75 \%$ of all moderate and severe brain injuries are cared for in 25 referral centres across Canada most serving a catchment area of 1.2 million. It is proposed that in areas where a comprehensive program for the brain injured has not been implemented, or is incomplete, this program or an appropriate modification of it be implemented and that these efforts be spearheaded by a concerned neurosurgeon in each referral centre. The plan includes a system of data collection which when gathered nationally, will permit ongoing assessments of current and proposed elements of the program.

13.

Changes in Arterior Ultrastructural Morphology Suggesting Post-Traumatic Vasospasm in the Spinal Cord

\section{D.L. ANTHES, E. THERIAULT and C.H. TATOR (Toronto, Ontario)}

Spinal cord trauma is a complex phenomenon involving both primary and secondary mechanisms of injury. The primary traumatic insult initiates a variety of secondary processes that enhance injury severity during the acute postinjury phase. Amony these secondary processes, ischemia is particularly prominent. While several mechanisms of ischemia following spinal cord trauma have been hypothesized (vessel rupture, shearing, compression, intravascular thrombosis), vasospasm has not been convincingly characterized. The present study provides quantitative anatomical evidence for enhanced vascular tone and reductions in arteriolar cross-sectional area following experimenatl spinal cord injury. Nine adult female Wistar rats received a $51 \mathrm{~g}$ clip compression spinal cord injury at the C8-T1 level following a cervical laminectomy (C7-T2) under halothane 
anesthesia. Three animals were sacrificed at each postinjury survival time: $15 \mathrm{~min}, 2 \mathrm{hrs}$ and $24 \mathrm{hrs}$. Three additional sham control rats were sacrificed 24 hrs postoperatively. Following transcardial aldehyde perfusion, sulcal arterioles from within the ventral median fissure were cut in cross-section in the coronal plane midway along the fissure at the injury site and prepared for electron microscopy. For each sulcal arteriole, all smooth muscle cells were quantitatively analyzed in a blinded manner on an IBAS image analysis system for length and width. Arteriolar luminal cross-sectional area was also measured. A clear decreasing trend was observed for luminal cross-sectional area with time and very marked and statistically significant changes in smooth muscle cell dimensions were observed. Smooth muscle cell length was dramatically reduced $(p=0.001)$ and width dramatically increased $(p=0.001)$ postinjury when compared to preinjury dimensions. Smooth muscle cell contraction has been clearly characterized in vitro to involve these specific morphometric alterations: decreased length accompanied by increased width. The reductions in luminal cross-sectional area correlate directly with the constrictive changes measured in the smooth muscle cells. The results of this study support the notion of enhanced vascular tone ("vasospasm") following acute spinal cord injury.

\section{4.}

Radiofrequency Lesions in the Dorsal Root Zone for Treatment of Persistant Pain Secondary to Traumatic Injuries of the Terminal Spinal Cord

\section{J.H. SAMPSON, R.E. CASHMAN and B.S. NASHOLD (Durham, U.S.A.)}

Radiofrequency lesions in the dorsal root entry zone (DREZ lesions) have been shown to relieve the majority of patients with pain secondary to paraplegia. DREZ lesions, however, have not been as successful in treating pain from peripheral nerve injuries. Traumatic lesions of the conus medullaris and cauda equina are unique in that both the spinal cord and peripheral nerve roots are injured. Pain syndromes from such injuries are notoriously intractable and the risks of ablative surgery are greater because neurologic deficits are often incomplete. This report discusses our experience with DREZ lesions used to treat intractable pain in 39 patients with trauma to the terminal spinal cord.

The group includes 29 patients with primarily conus medullaris lesions (spinal level T12-L1) and 10 patients with cauda equina lesions (spinal level L2-S1). Gunshot wounds were the most common cause of the injury (21/39). Patients with conus medullaris injuries tended to have a later onset (18 months) of "diffuse-burning" pain and $45 \%$ were paraplegic. In contrast, patients with injuries to the cauda equina had an earlier onset (1 month) of "electrical-shooting" pain which was usually unilateral and radicular, and most had incomplete neurologic deficits.

Pain relief was similar in both groups. At 3-year follow-up, $54 \%$ were pain free without medications, and $21 \%$ had improvement in their pain, but still required non-narcotic analgesics. Better outcomes were noted in those patients with conus medullaris injuries and with incomplete neurologic deficits.
There were no complications in the cauda equina group. In the conus medullaris group, 2 had post-operative CSF leaks, and 4 had increased weakness, and, of these, 1 experienced loss of bladder function, and one loss of orgasm.

This preliminary report suggests that DREZ lesions may be useful in combating intractable pain from traumatic injuries to the terminal spinal cord.

15.

Increased Interictal Spiking and Surgical Outcome After Selective Amygdalo-Hippocampectomy

F. CENDES, F. DUBEAU, F. ANDERMANN, A. OLIVIER, A. CUKIERT, E. ANDERMANN and L.F. QUESNEY (Montreal, Quebec)

We studied the electrocorticogram (ECoG) before and immediately after transcortical selective amygdalo-hippocampectomy, prospectively in 12 consecutive patients and retrospectively in 3 others (mean age 30.8 years). Mean postoperative follow-up was 19 months (range 5 - 87). ECoG was performed with surface and two depth electrodes inserted through T2, at 3 and 4.5 $\mathrm{cm}$ from the temporal pole. Before resection the ECoG showed a variable amount of interictal spiking, recorded either independently from the depth and surface, or synchronously. A small cortical incision $(2-3 \mathrm{~cm})$ was made in T2. The hippocampus, amygdala and parahippocampal gyrus were removed subpially. After resection increased epileptiform abnormality was observed in all 15 patients and a different ECoG pattern emerged. It consisted of repetitive, high amplitude spikes and polyspikes, separated by attenuated background, recorded from the most anterior temporal area. Nevertheless, the outcome was comparable to that of standard anterior temporal resection: $73 \%$ class 1 and $13.5 \%$ class 11 (Engel's scale). Similar observations were reported by Paulo Niemeyer (In: Baldwin and Bailey, eds. Temporal lobe epilepsy; Springfield 1958: 461-482).

ECoG is often used to tailor the amount of resection, and the persistence of epileptic abnormalities correlates with worse outcome. This is not the case in selective amygdalo-hippocampectomy, suggesting a different underlying mechanism for the increased interictal spiking following this procedure.

\section{6.}

Evolution of the Role of the Amygdala in Temporal Lobe Seizures with Automatism and Amnesia

\section{W. FEINDEL (Montreal, Quebec)}

Surgical resection of the temporal lobe for treating seizures has been applied since the early 1930s. But the significance of the mesial temporal structures in seizures characterized by automatism and amnesia became evident only in the early 1950 s, mainly from studies of surgical findings, including stimulation of the amygdala to evoke features of automatism and its corticographic expression, localization of surgical pathology and correlation of the pattern of surgical removal with postoperative 
seizure control and neuropsychological evaluations. Further confirmation of the significance of amygdala ablation is provided from analysis of 200 patients operated on consecutively for focal termporal lobe seizures and followed from 2 to 20 years. Two different patterns of surgical resections were reviewed: 100 cases with anterior temporal corticectomy and removal of amygdala and minimal hippocampal excision and another 100 cases with additional major-excision of hippocampus. Surgical outcome in regard to seizure control and evaluation of cognitive function showed no significant difference between these two groups. Details of the extent of surgical resection will be presented from anatomical and MRI studies. The results indicate that anterior temporal lobectomy including the amygdala can offer seizure relief without the risk to memory function attendant upon excision of the hippocampal formation.

\section{7.}

The Evolution of Functional Hemispherectomy at the Montreal Neurological Institute

\section{C.R. MASCOTT, W. CHOI, T. RASMUSSEN and J.-G. VILLEMURE (Montreal, Quebec)}

Hemispherectomy for intractable seizures in patients with infantile hemiplegia and other hemispheric pathologies became popular in the 1950s and 1960s due to the excellent relief of seizures provided. Superficial cerebal hemosiderosis $(\mathrm{SCH})$, a late complication of anatomical hemispherectomy, led to the disuse of this operation. At the Montreal Neurological Institute, functional hemispherectomy developed out of multi-staged procedures. In its present form, the functional hemispherectomy involves a complete disconnection of the pathological hemisphere. This is accomplished by a complete callosotomy, section of the corona radiata, central area removal, and disconnection or removal of the temporal lobe. On the basis of surgical and anatomical dissections, aspects of functional hemispherectomy technique are discussed.

Callosotomy from within the lateral ventricle, extent of amygdalar removal, and method of temporal disconnection or removal are all integral parts of this procedure. A properly performed functional hemispherectomy should provide the same benefits in seizure relief as an anatomical hemispherectomy while eliminating the high tardive morbidity and mortality of the earlier anatomical variants. At the MNI, a total of 37 functional hemispherectomies have been performed to date. No cases of SCH have been noted. In $94 \%$ of patients, seizures have subsided postoperatively either very signficantly (over $80 \%$ reduction) or completely.
18.

Angiographic Screening and Elective Surgery of Familial Cerebral Aneurysms: A Decision Analysis

\section{R. LEBLANC, K.J. WORSLEY, D. MELANSON and D. TAMPIEREI (Montreal, Quebec)}

Background: Up to $8 \%$ of cerebral aneurysms may be familial. We established the prevalence of aneurysms in the second generation of individuals with familial cerebral aneurysms, and determined their proper management by decision analysis.

Methods: The members of the second generation of four families in whom a mother and child had a ruptured aneurysm underwent elective cerebral angiography. The prevalence of aneurysms in the second generation $(\mathbf{P})$ was thus established at $29.4 \%$. We estimated $2 \%$ as the annual risk of rupture $(\mathbf{R})$, $72.7 \%$ as the risk of death or disability (M) with rupture, $0.1 \%$ as the risk of angiography (A) and $6.5 \%$ as the risk of surgery (S). The benefit in years of survival free of sequelae resulting from angiographic screening and elective surgery (intervention) over natural history was computed from the equation

$$
\mathrm{L}\left(\left\{\mathrm{P}\left[1-(1-\mathrm{R})^{\mathrm{L}}\right] \mathrm{M} / 2\right\}-\{[\mathrm{A}]+[(\mathrm{P} \cdot \mathrm{S})(1-\mathrm{A})]\}\right)
$$

for life expectancy $(\mathbf{L})$ corresponding to each quinquennial age group from age 15 to 100 years.

Results: Intervention produces a gain of at least one year of survival free of sequelae for patients whose life expectacy is 32 years or more, corresponding to an age of 53.5 years for females and 49 years for males. Greater benefit is achieved with increasing life expectancy.

19.

Early Clinical Experience with Guglielmi Detachable Coils (GDC) for Treatment of Subarachnoid Aneurysms: 15 cases

D. ROY, J. RAYMOND, G. L'ESPERANCE, M.W. BOJANOWSKI and S. FONTAINE (Montreal, Quebec)

From August 22 to December 22, 1992, 15 patients ( 9 females, 6 males, mean age 56.2) were admitted on the F.D.A. approved protocol supervising the use of GDC. Nine patients were treated for subarachnoid hemorrhage (SAH) and 6 patients for an unruptured aneurysm. Three patients were treated more than once for a total of 18 procedures. There was one failed attempt to catheterize the aneurysm, leaving 14 treated patients. The initial percentage of aneurysmal occlusion was $85-95 \%-$ 3 cases, $95-98 \%-2$ cases, $>98 \%-8$ cases. One case could not be assessed because of an aneurysmal rupture during the procedure leading to a fatal evolution. This was the only fatality of the series. A 3-month follow-up angiogram is available for 6 patients. There was a minimal reopacification of the neck of the aneurysm in 4 cases. Two of these patients were retreated with more than $98 \%$ occlusion. There were 2 other cases of perforation and leakage without permanent consequences. Three patients presented TIAs with complete recovery. Recent clinical follow-ups for the SAH group show: 7 complete recoveries without rebleeding, 1 death and 1 failure of treatment who 
underwent surgery. In the unruptured group, all patients are in the same clinical condition as prior to treatment. The mean clinical follow-up is 3.5 months. Six-month follow-up angiograms should be available for all patients at the time of this presentation.

20.

\section{Spin Angiograms: Role in Assessing Intracranial Aneurysms}

\section{D.J. HOFF, K.G. TERBRUGGE, C. WALLACE and F. GEN- TILI (Toronto, Ontario)}

We retrospectively and prospectively reviewed over 50 angiograms comparing a spin angiogram technique to high quality conventional angiograms. The spin angiogram is performed by rotating an L-U arm 180 degrees around the patient while injecting contrast media in order to obtain a real time dynamic angiography study. Patients were studed both pre- and postsurgery for aneurysms. In $25 \%$ of the post-operative studies, we were able to see a residual neck not seen on the conventional angiogram.

In the pre-operative patients the spin angiogram provided significant additional information in regards to the location, neck, branch anatomy and projection. In three patients a single aneurysm, (or an additional aneurysm among multiple aneurysms) was seen only on the spin angiogram.

\section{1.}

\section{The Role of Endovascular Treatment of Small Size Brain Arteriovenous Malformations}

\author{
K.G. TERBRUGGE, R.A. WILLINSKY, W. MONTANERA \\ and P. LASJAUNIAS (Toronto, Ontario; Paris, France)
}

Of 120 patients treated with embolization with tissue adhesive (cyanoacrylate) between 1985 and 1993, 22 patients harbored an arteriovenous malformation of the brain less than $3 \mathrm{~cm}$ in size. Fifteen of these patients presented with hemorrhage, 6 with seizures and 1 with headaches. With endovascular treatment 7 patients were cured, 7 were cured with embolization and radiotherapy and 6 patients still await further follow-up following the combined treatment. Two patients declined further treatment when embolization resulted in near complete obliteration of the embolization.

Conclusion: Endovascular treatment can have an important role in the treatment strategy of small size brain arteriovenous malformations.

\section{2.}

\section{Effect of Surgery on Severity of Vasospasm}

\section{R.L. MacDONALD, M.C. WALLACE and T.J. COYNE} (Toronto, Ontario)

Intracranial aneurysm surgery between 4 and 12 days after subarachoid hemorrhage (SAH) has been associated with increased risk of delayed cerebral ischemia and poor outcome compared to when surgery is performed before or after this time. To investigate if this increased risk is due to aggravation of vasospasm, cerebral arterial diameters were measured on angiograms of 56 patients operated on at various times after aneurysmal SAH. Aggressive surgical clot removal was not performed. To correct for differences in prognostic factors for vasospasm between patients operated at different times after $\mathrm{SAH}$, multiple regression analysis was performed using the arterial diameters during vasospasm as the dependent variable and propnostic factors for vasospasm, including the time when surgery was performed, as independent variables. Equations predicting severity of vasospasm could be generated using clinical grade on admission, age, and preoperative arterial diameter. Time of surgery had no effect on vasospasm. Cerebral infarction due to vasospasm in 6 of 30 patients $(20 \%)$ operated on within 3 days of SAH and in 4 of $15(21 \%)$ of patients operated on between 4 and 12 days after SAH ( $p=0.66)$. Good outcome for these 2 groups occurred in $88 \%$ and $85 \%$, respectively $(p=$ $1.00)$. These results suggest surgery has no effect on vasospasm. Any increased risk of cerebral ischemia which is associated with surgery 4 to 12 days after SAH is due to factors other than aggravation of vasospasm.

\section{PEDIATRIC NEUROLOGY}

23.

\section{Effect of Encephaloduroarteriosynangiosis (EDAS) on Outcome from Childhood Moyamoya Disease}

I.B. ROSS, J.L. MONTES, M.I. SHEVELL, J.-P. FARMER, B. ROSENBLATT and A.M. O'GORMAN (Montreal, Quebec)

Moyamoya disease is a rare, angiographically defined process in which there occurs bilateral progressive stenosis and often occlusion of the blood vessels of the anterior circulation. Cerebral ischemia can become clinically symptomatic during the active phase of the disease. While superficial temporal artery to middle cerebral artery anastomosis has been used successfully to treat moyamoya disease in the adult population, the procedure is technically difficult in children.

Encephaloduroarteriosynangiosis (EDAS), which involves the simple transportation of a segment of a scalp artery onto the surface of the brain, promotes the development of collateral blood flow in ischemic hemispheres. EDAS was performed on eight sides in six children with moyamoya disease. Clinical stabilization was noted in all children after the procedure. Postoperative angiography revealed evidence of blood flow to the brain via the EDAS in three of the six procedures that were studied. The two patients who did not demonstrate collateral blood flow via the EDAS were found to have angiographic regression of their disease. Clinical outcome was better after early intervention, before permanent deficits had developed. It is concluded that EDAS is a safe and effective procedure aimed at the prevention of ischemic neurological injury in children with moyamoya disease. 
24.

Near Infrared Spectroscopy: A New Technique for Monitoring Cerebral Oxygenation in Premature Newborns

M. MOODLEY, M. STEWART, E.H. ROLAND, B.A. LUPTON, A. MacNAB and A. HILL (Vancouver, British Columbia)

Near-infrared spectroscopy (NIRS) is a new, noninvasive technique which assesses cerebral oxygenation, bloodflow $(\mathrm{CBF})$ and blood volume (CBV). The purpose of this study was to monitor cerebral oxygenation and hemodynamics in premature newborns using NIRS during medical interventions, e.g., administration of surfactant, aminophylline and tracheal suctioning.

Twenty premature newborns with respiratory distress syndrome received surfactant during the first day of life. NIRS demonstrated no significant reduction in the level of oxyhemoglobin during, or immediately following surfactant administration. Twelve infants had increase in cytochrome oxidase levels which suggests improved cerebral tissue oxygenation. In 10 infants, who received aminophylline for treatment of apnea of prematurity, increased level of cytochrome oxidase and decreased CBV were noted. During tracheal suction, without preoxygenation, NIRS demonstrated rapid decline in cerebral oxyhemoglobin. In contrast, suctioning with preoxygenation resulted in increased cerebral oxyhemoglobin.

These data demonstrate that NIRS is useful for direct, continuous monitoring of cerebral oxygenation and hemodynamics in premature newborns during medical interventions. Administration of surfactant and aminophylline are not associated with adverse effects on cerebral oxygenation.

25.

Neurologic Complications of Cardiac Surgery in Children with Congenital Heart Disease

C.E. NIESEN, G. MILO-MANSON, M. GELATT, B.W. MCCRINDLE and W.J. LOGAN (Toronto, Ontario)

A 10-year retrospective medical record review was undertaken to describe the spectrum of acute neurologic complications in pediatric cardiac surgery. Procedures performed between Jan. 1982 and May 1992 were reviewed and patients with major congenital anomalies, prematurity or pre-morbid neurologic abnormalities were excluded. Over 120 patients were identified: $77 \%$ with seizures, $49 \%$ with strokes, $26 \%$ with seizures and stroke, and $4 \%$ with dyskinesia. These events occurred after pump surgery (cardiopulmonary bypass \pm aortic crossclamping) in about three-quarters of the patients. Ages ranged from 1 week to 13 years old (median: 7 months). Forty-five per cent of the events occurred within 24 hours of surgery; only $8 \%$ of the events occurred $>7$ days post-op.

Focal seizures occurred in $53 \%$ of seizure patients; hemmorrhagic infarcts occurred in $18 \%$ of stroke patients. Outcome at hospital discharge was variable. Twenty per cent of children had optimal outcome (no deficits or seizures), $25 \%$ had sub-optimal outcome (anti-epileptic drug (AED) or mild impairment) and $20 \%$ had poor outcome (severe motor, cognitive impairment or poorly controlled seizures). Thirteen per cent died in hospital. Though $57 \%$ of patients with seizures were discharged on AED, only $14 \%$ still required AED at follow-up of one year or more.

Increasing bypass time during pump surgery $(p<0.05)$ was associated with a poor outcome. Though seizures occurred in over $75 \%$ of our study population, epilepsy was not the main cause of neurologic morbidity at follow-up. A prospective casecontrol study to identify risk factors and frequency of neurologic sequelae in pediatric cardiac surgery is underway.

\section{6.}

The Importance of Early Angiography in the Investigation of Childhood Stroke

\section{E.P. WOOD and S. CHUANG (Toronto, Ontario)}

Cerebral arterial dissection is often not considered as a cause of stroke in children. Therefore angiography may not be included as an initial investigation. Promopt diagnosis is essential as anticoagulation is reported to improve outcome.

Angiographic reports of all children presenting with stroke to The Hospital for Sick Children (HSC) in Toronto from 1969 to 1992 were reviewed. In 30 cases vascular abnormalities compatible with dissection were reported. These angiograms were reviewed and 11 cases of cerebral dissection were confirmed. The patients charts were reviewed for clinical data.

Patients, 6 males and 5 female, ranged in age from $2-15$ years. Two-thirds reported recent minor to moderate trauma. The anterior circulation was involved in 6 and the posterior in 5 .

Most patients were admitted to HSC within 4 days of symptom onset. Angiography was usually within 2 days of admission but in several cases was delayed up to 11 days. Following diagnosis most patients received anticoagulants. One-half had major sequelae. There was a strong correlation between delay in diagnosis and poor outcome.

In conclusion, early diagnosis of cerebral artery dissection is essential to optimize outcome. Angiography is the only method of confirming dissection. Angiography should be performed early in the investigation of childhood stroke.

27.

Cerebral Blood Flow in Cryptogenic Lennox-Gastaut Syndrome

M. CONNOLLY, K.J. POSKITT and K. FARRELL (Vancouver, British Columbia)

Lennox-Gastaut syndrome (LGS) is associated with various underlying disorders including cerebral dysgensis, hypoxicischemic brain injury and CNS infections, but the pathophysiological basis of LGS is poorly understood. Unilateral areas of hypometabolism or hypermetabolism have been demonstrated by positron emission tomography in a small number of patients with LGS. We describe cerebral blood flow studies using 
Xenon-enhanced CT (XE-CT) and HMPAO-SPECT in 5 children with LGS.

The children were aged $4-12$ years and met the following criteria: tonic seizures recorded during video-EEG monitoring; slow background and $1.5-2.5 \mathrm{~Hz}$ spike wave complexes on the interictal EEG; and cognitive deterioration. CT and MR head imaging were normal in all children. Investigations did not demonstrate an underlying cause.

XE-CT was normal in 3 children and demonstrated abnormal cerebral blood flow in one frontal lobe in 2 children. HMPAOSPECT demonstrated abnormal blood flow in all children: unilateral frontal -2 ; unilateral hemisphere -1 ; bifrontal and unilateral temporal -2 .

The preliminary data from this on-going study demonstrates that focal cerebral blood flow abnormalities are common in children with cryptogenic LGS. This suggests that focal cerebral dysfunction may play a role in the pathophysiology of cryptogenic Lennox-Gastaut syndrome.

\section{8.}

\section{Clinical-Radiologic Correlates in Hemiparetic Cerebral Palsy}

\section{P. HUMPHREYS and S. WHITING (Ottawa, Ontario)}

Thirty-two consecutive children with hemiparetic cerebral palsy were assessed using a standardized questionnaire and examination protocol; all received a head CT scan to assess the extent of neuropathologic changes. The most important $\mathrm{CT}$ findings included: infarction or gyral abnormalities in the distribution of a major cerebral artery ( 8 pts.), porencephaly ( 7 pts.), periventricular leukomalacia (11 pts.), diffuse hemispheric atrophy ( 3 pts.), focal cystic lesions of deep white matter ( 3 pts.) or central grey structures ( $4 \mathrm{pts}$ ); some patients had more than one finding. Seventeen patients (53\%) had significant cortical grey matter pathology on CT; the remaining 15 patients had radiologic changes confined to deep white matter \pm central grey structures. Statistical analysis of the two groups showed no difference with respect to sex, side of hemiparesis, length of gestation, presence of intra-uterine growth retardation, gestational illness, perinatal asphyxia, or post-natal illness. The cortical grey matter pathology group were more likely to have a severe functional disability of the involved upper limb $(14 / 17$ vs. $3 / 15, p<0.005)$, learning disabilities or mental subnormality $(11 / 17$ vs $2 / 15, p<0.001)$ and recurrent afebrile seizures $(13 / 17$ vs. $0 / 15, p<0.001)$. The central white matter group were more likely to show predominant involvement of the lower limb $(8 / 15$ vs. $2 / 17, p<0.005)$. These results suggests that neuroradiologic findings in hemiparetic cerebral palsy may be of some prognostic value with regard to pattern of disability, presence of learning difficulties, and the development of epilepsy.
29.

\section{Routine Surveillance Imaging of Children with Shunted Hydrocephalus}

\author{
R.W. GRIEBEL and A. WOODARD (Saskatoon, \\ Saskatchewan)
}

CT scanning and ventricular ultrasonography are commonly used in the follow-up of children with shunted hydrocephalus. It has been our practise to carry out routine ultrasonography or CT scanning of all shunted children within the first year of shunt placement. A retrospective review of these post-op studies was carried out to determine how the studies influenced subsequent management of the children.

Over a 4-year period 65 children between ages of 1 day and 5 years underwent CSF shunting at Royal University Hospital, Saskatoon. During the year subsequent to the procedure $42 \mathrm{CT}$ scans and 22 ultrasound studies were carried out on a routine basis. All children were asymptomatic at the time of the studies. Seventy-eight per cent of the routine CT studies showed normal or collapsed ventricles, while $19 \%$ were mildly enlarged. One study demonstrated persistent marked ventriculomegaly which prompted surgical intervention. None of the ultrasound studies showed moderate or marked ventricular enlargement, and no surgical intervention resulted from these studies. None of the children with mild ventriculomegaly required shunt revisions over the 6 months following CT assessment.

We conclude that routine imaging studies of the asymptomatic shunted child seldom provides information which alters management of the child.

\section{0.}

Electroencephalogram Results are Rarely the Same When Repeated Within 6 Months in a Child with Epilepsy

\section{P.R. CAMFIELD, C.S. CAMFIELD, K. GORDON, J.M. DOO- LEY and B. SMITH (Halifax, Nova Scotia)}

Epilepsy classification depends on the EEG. The reliability of this important test was assessed by examining EEG results of all 506 children diagnosed in Nova Scotia with epilepsy between 1977 - 1985 (excluding minor motor and absence). One hundred fifty-nine children had an EEG at the time of diagnosis (EEG1) plus a second EEG (EEG2) within 6 months. EEG2 was more likely ordered if EEGI was normal and less likely if EEG I contained sleep $(p<0.05)$. Thirty-four per cent of EEGI and $26 \%$ of EEG2 were normal (both normal in 14\%). If EEGI was abnormal, $40-70 \%$ of each type of abnormality was not present on EEG2. Analysis of the 67 cases abnormal on both EEGI and EEG2 follows. Of 42 with focal spikes and/or focal slowing on EEG 1, 7 had only generalized spike wave on EEG 1, 7 showed only major focal abnormalities on EEG2.

Formal statistical testing for reliability showed low Kappa scores indicating low reliability. We conclude that the EEG in childhood epilepsy is an unstable measure. A repeat EEG within 6 months of a first EEG may yield conflicting information. 
31.

\section{EEG Patterns in Comatose Children}

\section{J. WOLSTEIN, J.D. REGGIN and S.S. SESHIA (Winnipeg,} Manitoba)

Our objectives in this study were: (i) define EEG patterns in comatose children, and (ii) determine the applicability of the Hockaday Classification (1965).

Fifty-five EEGs were done on 24 comatose children aged 0.2 to 18 years (median 2.1 years) between January 1990 and July 1991. The EEGs were analyzed visually. The EEG patterns were: Intermittent attenuation (29\%), hemispheric asymmetry $(29 \%)$, isolated spikes or sharp waves (29\%), high amplitude slow $(27 \%)$, burst - suppression $(24 \%)$, spindles $(22 \%)$, persistent regional or generalized attenuation (19\%), electrographic seizures $(15 \%)$, EEG-clinical seizures $(15 \%)$, rhythmic focal or generalized delta activity (13\%), PLEDS (9\%), ECS (7\%), monomorphous biphasic waves (4\%), and triphasic waves $(2 \%)$. More than one pattern was found in 49/55 (89\%) EEGs. The patterns changed through all serial records ( $n=42,11$ patients). The Hockaday Classification could not be readily applied in 12/55 (22\%) EEGs because of hemispheric asymmetry, epileptiform activity, or multiple abnormalities, features not incorporated in the Classification.

Conclusions: Previous descriptions have generally concentrated on individual patterns. Our study shows that multiple patterns may occur in the same record. The Hockaday Classification can only be applied with reservations.

\section{2.}

Open Label Study of Vigabatrin in Refractory Partial Epilepsy and Infantile Spasms

\section{CONNOLLY, R. MUNN, M. BERG and K. FARRELL} (Vancouver, British Columbia)

Vigabatrin, a specific inhibitor of GABA-aminotransferase, has been demonstrated to be effective in the treatment of certain seizure types. We describe the efficacy and tolerability of vigabatrin in an open-label, add-on study in 25 children, aged 10 months to 19 years (mean 7.5 years). Seizure types included infantile spasms (8) and partial seizures (17). The seizures had been refractory to $3-13$ anti-epileptic drugs. The underlying etiology in 13 children was cerebral dysgensis (6), tuberous sclerosis (4), and hypoxic-ischemia (3).

The initial vigabatrin dose was $25 \mathrm{mg} / \mathrm{kg} /$ day and the dose was increased by $25 \mathrm{mg} / \mathrm{kg} /$ day at three-week intervals up to $150 \mathrm{mg} / \mathrm{kg} /$ day. The final dose ranged from $25-150 \mathrm{mg} / \mathrm{kg} / \mathrm{day}$ (mean $80 \mathrm{mg} / \mathrm{kg} /$ day). Mean follow-up was 6.3 months $(2-13$ months). The change in seizure frequency is described below:

\begin{tabular}{lcccc}
\hline & Sz free & $\mathbf{2 0 0 \%}$ dec & $\mathbf{5 0 - 9 0 \% \text { dec }}$ & $<\mathbf{5 0 \% \text { dec }}$ \\
\hline \hline Spasms (8) & 0 & 1 & 1 & 6 \\
Partial (17) & 1 & 0 & 3 & 13 \\
\hline
\end{tabular}

Adverse effects were reported in 9 children: change in behavior (3), sedation (4), drooling (1), weight gain (1). Vigabatrin resulted in a $>50 \%$ reduction in seizure frequency in $25 \%$ of children.

33.

\section{EP Abnormalities in Children with Neurofibromatosis}

\author{
N.K. KEENAN, M.J. TAYLOR and W.J. LOGAN (Toronto, \\ Ontario)
}

Neurofibromatosis type I is a dominantly inherited disorder that affects cell growth of neural tissue and can lead to tumor growth on nerves at any location. There is considerable variability in disease manifestation. Multimodal evoked potential studies provide a quantitative evaluation of function of systems which could help in diagnosis and follow-up management of these patients. Brainstem auditory, visual and somatosensory evoked potentials (ABR, VEP, SEP) were recorded in 29 children with neurofibromatosis type I, between 10 months and 19 years (mean 8.5 years.).

Twenty-one of $29(72 \%)$ patients had complete evoked potential (EP) studies. Two of these $21(10 \%)$ had normal EP studies. In the others, abnormalities were found as delays in BAEPs $(57 \%)$, VEPs (54\%), and SEPs (39\%). In most of these cases there was no clinical or imaging evidence of tumor growth. The high rate of abnormal studies indicates that multimodal EPs are useful for screening children with neurofibromatosis, as clinically undetectable abnormalities can be found. Some may represent tumor formation, but others probably indicate non-neoplastic local areas of brain dysplasia. With a larger series with long-term follow-up, the significance of these EP abnormalities and the value of monitoring patients with neurofibromatosis will be determined.

\section{NEUROLOGY}

34.

\section{The Fate of Schwann Cells in Nerve Allografts}

\section{R. MIDHA and S.E. MacKINNON (Toronto, Ontario)}

Purpose: To investigate the phenotype of Schwann cells in the allograft nerve segment under conditions of no, temporary, or continuous immunosuppression.

Methods: Nerve transplants were performed from mutant Shiverer mice donors, whose Schwann cells lack myelin basic protein (MBP), to normal wild-type recipients. Forty normal BALB/c mice $\left(\mathrm{H}-2^{\mathrm{d}}\right)$ were engrafted with $1 \mathrm{~cm}$ sciatic nerve grafts from allogeneic $\mathrm{C} 3 \mathrm{H} / \mathrm{eb}\left(\mathrm{H}-2^{\mathrm{k}}\right)$ Shiverer mice. Allografted mice received either no, temporary (6 weeks), or continuous (14 weeks) immunosuppression, using cyclosporin A (CsA) at s.c. doses of $20 \mathrm{mg} / \mathrm{kg} /$ day. Following sacrifice at 6 , 10 or 14 weeks, the host and grafted nerves were removed and immunostained for MBP reactivity to unambiguously distinguish host from donors Schwann cells. 
Results: The results of MBP reactivity are summarized below, where the number of MBP positive graft segments per number of mice studied are noted in each cell:

\begin{tabular}{lccc}
\hline Groups & Week 6 & Week 10 & Week 14 \\
\hline \hline No CsA & $3 / 5$ & $5 / 5$ & $5 / 5$ \\
Temp CsA & - & $4 / 5$ & $4 / 4$ \\
Con CsA & $0 / 5$ & $2 / 5$ & $2 / 4$ \\
\hline
\end{tabular}

With no immunosuppression, host derived MBP-positive Schwann cells replaced rejected donor Schwann cells in the graft. A similar process occurred under temporary immunosuppression, albeit in a delayed fashion. Even with continuous immunosuppression, replacement of donor by recipient Schwann cells was observed over time in approximately onehalf the subjects.

Conclusions: In short nerve allografts, host-derived Schwann cells progressively replace donor Schwann cells. This study provides a scientific basis for the need of only finite host immunosuppression in conditions of clinical nerve allografting.

35.

\section{Pain in Guillain-Barre Syndrome}

D.E. MOULIN, R. AMIREH and A.F. HAHN (London, Ontario)

We conducted a prospective study of pain in Guillain-Barre Syndrome (GBS) to determine its prevalence, intensity, character and response to treatment. Twenty-three consecutive patients with GBS were followed for a mean of 15.6 weeks. On admission 12 of 23 patients (52.2\%) described pain that was either distressing, horrible or excruciating (Present Pain Intensity, McGill Pain Questionnaire) and only 3 patients had no pain. The mean pain intensity using a visual analogue scale (VAS, 0 $10 \mathrm{~cm}$ ) was 5.42 (SD 3.41). Pain was localized to back and legs and was almost always worsened by nerve root traction manoeuvres such as straight leg raising. Sixteen patients required narcotic analgesics with 8 receiving parenteral morphine. Mean pain relief (VAS, $0-10 \mathrm{~cm}$ ) was $6.8,7.3,8.0$ and 9.6 at 2, 4, 8 and 16 weeks respectively. Acute back and leg pain of a neuropathic nature is a major feature of GBS and requires aggressive treatment.

36.

Autonomic Cardiovascular Reflex Abnormalities in Diabetics with Somatic Peripheral Neuropathy

J.D. STEWART, J. GOTMAN, V. MARCUS and S. LAW (Montreal, Quebec)

Objective: To evaluate the incidence of cardiovascular autonomic abnormalities in diabetics with somatic neuropathy, and the relative involvement of parasympathetic and sympathetic systems.

Methods: Beat-beat blood pressure (BP) and heart rate (HR) were recorded from digital arteries using an Ohmeda Finapres.
Responses to deep breathing, lying-standing, Valsalva manoeuver were evaluated in 34 diabetics with moderate or severe somatic peripheral neuropathy and compared with controls.

Results: Only HR (mainly parasympathetic) abnormal 12 (35\%); only BP (sympathetic) abnormal $4(12 \%)$; both abnormal $13(38 \%)$; normal responses $5(15 \%)$.

Conclusions: Cardiovascular autonomic reflex abnormalities occur variably in diabetics with somatic neuropathy. The parasympathetic or sympathetic system can be involved separately, or together, or neither may be abnormal. Contrary to many published reports, the sympathetic system can be abnormal even when the parasympathetic is normal.

37.

The Inhibition of Neurite Outgrowth by Rat and Human Myelins

M.H. SCHMIDT, A.M. LOZANO, C.C. RODER and A. ROACH (Toronto, Ontario)

Recent experiments have suggested that components of adult mammalian central myelin may inhibit the elongation of neural processes and thus restrict CNS axonal regeneration. We have developed an assay to study the influence of different myelins on neurite outgrowth in vitro. NG 108-15, a hybrid cell with neural properties was plated onto equivalent amounts of proteins $\left(20 \mu \mathrm{g} / \mathrm{cm}^{2}\right)$ from rat and human CNS myelin, rat PNS myelin and rat muscle. Neurite outgrowth was determined at 24 hours. Only cells with a process longer than 1 cell diameter were counted. Whereas $64 \pm 5 \%$ of cells on a control polylysine surface bore neurites only $5.8 \pm 9.3 \%$ of cells did so on rat CNS myelin and $9.4+12.8 \%$ on human CNS myelin $(\mathrm{p}<0.0005, \mathrm{n}=$ 16). On rat PNS myelin $41.9 \pm 22.7 \%$ had processes, on rat muscle $48.3 \pm 15.5 \%$. The results indicate that both rat and human CNS myelin are inhibitory substrates for neuritic growth. Rat PNS myelin is also inhibitory but to a lesser extent than CNS myelin. These observations correlate with the differences in in vivo regeneration between the CNS and PNS. The assay system is useful to characterize neurite-substrate interactions.

Supported by: Medical Research Council of Canada and Networks of Centres of Excellence.

38.

Chronic Inflammatory Demyelinating Polyneuropathy (CIDP) in the Setting of Autoimmune Glomerulonephritis

\section{C.J. WATLING, A.F. HAHN, T.E. FEASBY and M.G. ELLEKER (London, Ontario; Calgary; Edmonton, Alberta)}

CIDP has been reported in association with a variety of autoimmune diseases. We have observed a chronic progressive demyelinating polyneuropathy in 5 patients with glomerulonephritis. Renal biopsies showed definite or probable membraneous glomerulonephritis in 3 cases and rapidly progressive crescentic glomerulonephritis in the other 2 cases, 1 of which had typical clinical findings of Goodpasture's syndrome. 
Progressive neuropathic symptoms, moderate to severe weakness and numbness of the extremities, develop in these patients over periods ranging from 1 to 6 months. All underwent electrophysiologic studies and demonstrated abnormalities consistent with a diagnosis of CIDP, as outlined by Cornblath et al. (1991). CSF protein was elevated in 3 of 4 patients and the diangosis was supported by nerve biopsy in 2 patients. All patients were treated with Prednisone; a series of plasma exchanges were performed in 2 and 1 received intravenous human IgG. All improved neurologically. The etiology of CIDP remains unknown, but immune mechanisms are suspected to play a role. The renal lesions of autoimmune glomerulonephritis are mediated by glomerular deposition of immune complexes. An occurrence of a CIDP like neuropathy in this setting would lend support to a putative role of humoral factors in the pathogenesis of CIDP.

Cornblath et al., Neurology 41: 617-618, 1991.

39.

An in vitro Muscle Preparation for Investigating the Protective Effect of Mild Hypothermia in Ischemic Cell Death

V.A. CWIK, R. MAJUMDAR, J. SCHULLER and M.H. BROOKE (Edmonton, Alberta)

The experimental basis for the treatment of stroke is being widely explored. The use of NMDA blockers, of free radical scavengers and, recently, of mild hypothermia promises to revolutionize treatment. The effect of mild hypothermia (a drop of $2^{\circ} \mathrm{C}$ ) has a dramatic effect on preventing neuronal damage in experimental stroke. The mechanism of this effect is unknown. Brain tissue is inherently difficult to study, particularly when trying to correlate biochemical changes with tissue function. We have now shown that experimental ischemic damage to muscle in vitro exhibits the same temperature sensitivity. This may provide a simpler model for exploring the mechanisms of cell death.

Methods: Intact rat epitrochlearis muscles are suspended in Kreb's Ringer. Forty $\mu \mathrm{M}$ DNP, a mitochondrial uncoupler, is added. $\mathrm{PGE}_{2}$ and lactate release and the contractile response to electrical stimulation are measured and compared to controls. The experiments are performed at $37,35,33$ and $27^{\circ} \mathrm{C}$.

Results: At $37^{\circ} \mathrm{C}$, DNP-treated muscle releases large amounts of $\mathrm{PGE}_{2}$, an arachidonic acid metabolite and indicator of membrane injury. The process is calcium dependent. It is associated with failure of contraction and generation of lactate, typical of ischemic muscle damage. At $35^{\circ} \mathrm{C}$, this DNP-stimulated $\mathrm{PGE}_{2}$ release is completely inhibited. Contractile force is improved. Lactate production is still brisk indicating that the ischemic stress is still present.
40.

\section{Oculographic Analysis of Tullio's Phenomenon}

\section{W.A. FLETCHER (Calgary, Alberta)}

Tullio's phenomenon consists of paroxysmal sound-induced vestibular symptoms and nystagmus. The neural signals may originate in a semicircular canal or otolith organ. Studies in animals have shown that stimulating a single vestibular organ causes dysconjugate eye movements. To determine whether the same is true for humans, we recorded three-dimensional eye movements in three patients during Tullio's phenomenon, using a magnetic search-coil system. The effects of head tilt, convergence and changing stimulus duration were examined in two patients. All three patients responded maximally to a $2,000 \mathrm{~Hz}$ tone at $110 \mathrm{~dB}$ in one ear, and showed a combined upward-contratorsional movement of both eyes. Horizontal movement was absent. Brief tones provoked monophasic eye movements (velocities: 7 - $32 \mathrm{deg} / \mathrm{sec}$ ). Sustained tones provoked nystagmus with slow phases of $0.5-11 \mathrm{deg} / \mathrm{sec}$. In two patients, the ipsilateral eye had greater upward component velocities than the contralateral eye (ratios: 1.15 and 1.39). In the other patient, the reverse was true (ratio: 0.42 ). Ipsilateral head tilt of $20 \mathrm{deg}$ caused a $50 \%$ increase in the ratio of torsional to vertical velocities of the ipsilateral eye and a decrease of $\approx 20 \%$ in torsional velocity of the contralateral eye. Convergence had no constant effect on evoked eye movements. These results suggest that utricular stimulation causes Tullio's phenomenon and that in humans, utricular stimulation produces dysconjugate eye movements.

41.

Otolithic and Proprioceptive Influences on Human Torsional Vestibulo-Ocular and Optokinetic Reflexes

\section{J.A. SHARPE and M.J. MORROW (Toronto, Ontario)}

Effects of otolith input on the torsional vestibulo-ocular reflex (TVOR) and torsional optokinetic nystagmus (TOKN) in humans were unknown.

Methods: Otolithic and proprioceptive modulation of the TVOR were measured at $0.25,0.5$ and $1.0 \mathrm{~Hz}$ in 5 normal subjects under 3 conditions: (i) with the head and trunk erect, (ii) with the head supine and the trunk erect, and (iii) with the head and trunk supine. TOKN drum speeds were $10,20,40$ and 80 $\mathrm{deg} / \mathrm{sec}$. Torsional head and eye motion were recorded by magnetic search coils.

Results: During active head movements, TVOR gain was significantly higher with the head upright $(0.61 \pm 0.17$ at 1.0 $\mathrm{Hz})$ than with the head supine $(0.38 \pm 0.16)$, indicating that dynamic otolithic inputs can supplement the semicircular canalocular reflex. During passive earth-vertical axis rotation (head supine) TVOR, gain was similar with the head and trunk supine than with the head supine and trunk erect, implying that proprioceptive information from the neck and trunk has little effect upon the TVOR. TVOR gain was not increased by active, selfgenerated head movement compared to passive whole body 
rotation, indicating that the TVOR is not augmented by dynamic proprioceptive inputs or by efference copy of a command for neck movement. TOKN evoked by a full-field stimulus had a mean slow phase gain of 0.22 for $10^{\circ} / \mathrm{sec}$ drum rotation, but gain fell to 0.06 for $80 \% \mathrm{sec}$ stimuli. Mean TOKN slow phase velocity did not exceed $5 \%$ sec and after nystagmus was typically absent. TOKN was not modified by otolith or proprioceptive changes caused by altering head and trunk position with respect to gravity. Torsional velocity storage is negligible in humans, regardless of head orientation.

42.

\section{Latent Nystagmus: Waveforms, Pursuit and Motion Perception}

A.G. HUAMAN, J.J.S. BARTON and J.A. SHARPE (Toronto, Ontario)

Latent nystagmus (LN) develops in both eyes during monocular viewing; the fast phase is in the direction of the viewing eye. $\mathrm{LN}$ is identified by a decelerating slow phase velocity waveform. Strabismic patients with $\mathrm{LN}$ have nasal-temporal pursuit asymmetries, and motion perception deficits are proposed as a cause (J Neurosci 1986; 6: 2495).

We studied five LN patients with a magnetic search coil technique. We assessed slow phase velocity waveforms, determined mean slow phase eye velocity during fixation, and recorded pursuit eye movements to constant velocity target motion. Motion perception was measured by coherent dot motion and thresholds were determined for nasally and temporally directed motion.

The predominant slow phase velocity waveform was linear in 3 of 5 patients. Nasally directed pursuit was better than temporal pursuit, and in 4 of 7 eyes tested, the asymmetry was not accounted for by the additive effect of the LN slow phase on nasally directed pursuit. Directional deficits in motion perception signals did not correlate with the pursuit asymmetries in LN.

We conclude that the predominant $L N$ waveform is linear. The pursuit asymmetry in LN can not be explained by the effect of the LN slow phase upon nasal pursuit. Motion perception asymmetries did not correlate with pursuit asymmetries. Our results suggest that the defect in $\mathrm{LN}$ is downstream from the middle temporal visual area.

43.

Intravenous Anticholinergic Drugs in Downbeat and Pendular Nystagmus

J.J.S. BARTON, A.G. HUAMAN and J.A. SHARPE (Toronto, Ontario)

Seven patients with oscillopsia underwent a double-blind trial of 3 anticholinergic drugs: $0.4 \mathrm{mg}$ scopolamine, $2 \mathrm{mg}$ benz- tropine, and $0.2 \mathrm{mg}$ glycopyrrolate. The first two agents are centrally active but the last is not. Five patients had pendular nystagmus and 2 had downbeat nystagmus. We used magnetic search coils to record eye movements before and after drug administration and analyzed data for mean slow phase velocity of downbeat nyustagmus and mean peak velocity and mean amplitude of pendular nystagmus. We recorded Snellen visual acuity, a motion discrimination score on a random-dot clinematogram test, and a subjective oscillopsia score. Mean differences between before and after data were calculated for all parameters and t-tests were applied to group data. Scopolamine markedly reduced nystagmus in 6 patients, including all 5 with pendular nystagmus. Benztropine was moderately effective but glycopyrrolate had negligible impact. Visual acuity was only slightly better with scopolamine but motion discrimination improved significantly with scopolamine and, to a lesser degree, benztropine. Oscillopsia scores changed similarly.

We conclude that scopolamine and benztropine, centrally active anticholinergics, are more effective than glycopyrrolate, which does not cross the blood-brain barrier.

44.

The Effects of Edrophonium on Eyelid Saccades

F. ALTOMARE, J.J.S. BARTON, A.G. HUAMAN and J.A. SHARPE (Toronto, Ontario)

Edrophonium's effect on the dynamics of upward lid saccades ("laccades") has not been previously measured. We used magnetic search coil oculography to study two patients with myasthenia gravis, one patient with a compressive third nerve palsy, and one with chronic progressive external ophthalmoplegia.

Patients made saccades to a target stepping 20 degrees vertically from primary eye position at one-second intervals and received $10 \mathrm{mg}$ of edrophonium after four minutes. We plotted peak velocity against amplitude and derived the asymptotic peak velocity (Vmax). We measured the timing of the velocity peak during upward lid saccades as an index of intrasaccadic fatigue.

Edrophonium modified upward lid saccades only in the myasthenic patients. $V \max$ increased after edrophonium in both myasthenic patients, reflecting the presence of higher amplitude lid saccades. Linear regression analysis showed that an upward shift of the peak velocity/amplitude curve also contributed to this increase. In relation to total saccadic duration, velocity peaks occurred later after edrophonium in myasthenia.

Upward lid saccades in myasthenic patients attain larger saccadic amplitudes and can shift to a higher peak velocity/amplitude curve with edrophonium. Intrasaccadic lid fatigue also improved with edrophonium. 


\section{E. NEUROLOGY}

45.

\section{Isolated Amygdalar Sclerosis: Clinical Pathological Correlations}

\section{R.S. McLACHLAN, L.A. MILLER, M.S. BOUWER and L.P. HUDSON (London, Ontario)}

Mesial temporal sclerosis (MTS) implies cell loss and gliosis in both the hippocampus and amygdala. We have recently described isolated amygdalar sclerosis without hippocampal change in a subset of epilepsy patients following temporal lobectomy (Ann Neurol, in press). Clinical observations suggested that these patients might be distinguishable pre- and postoperatively from those with typical MTS. Two pathologically defined groups from a three-year series were studied; isolated amygdala sclerosis (AS) and combined amygdalar-hippocampal sclerosis (MTS). All 11 patients with AS were included and a comparison group of 20 MTS patients were randomly chosen. Mean follow-up of 20 months did not differ in the two groups. Only $28 \%$ (3/11) of AS patients became seizure free compared to $60 \%(12 / 20)$ of MTS patients. None of the AS patients had seizures before age 5 years compared to $80 \%$ of MTS patients. Auras were less frequent and fewer memory problems were documented in AS patients. MRIs were normal in all AS patients and abnormal in $80 \%$ of MTS patients. AS implies a poorer prognosis for complete seizure control following temporal lobectomy than does MTS. Furthermore, these two conditions may be distinguishable on the basis of the pre-operative clinical assessment.

\section{6.}

\section{Epilepsy and Pregnancy: Twenty Years' Experience}

E. ANDERMANN, I. LOPES-CENDES, L. DANSKY, M. OGUNI, M.H. SENI and F. ANDERMANN (Montreal, Quebec)

We have followed epileptic women for pre-pregnancy counselling and prospectively during pregnancy, and assessed the offspring of these women. Our aim was to establish a large cohort in order to analyze several parameters including: (i) the effects of anticonvulsant ( $A C$ ) regimen during pregnancy, on pregnancy outcome and post-natal neurological development, (ii) the impact of pre-pregnancy counselling; (iii) the effect of epilepsy on pregnancy, labour, delivery and puerperium, and (iv) changes in seizure pattern and $\mathrm{AC}$ pharmacokinetics during pregnancy.

A total of 319 patients, 105 of whom only for pre-pregnancy counselling was ascertained. The remaining 214 patients had 287 pregnancies resulting in 228 offspring, including 165 children who were followed after birth. The frequency of major malformations (MJ) in the offspring was increased as compared to the normal population ( $p<0.05$ ). Folate supplementation preor early during pregnancy was found to reduce MJ. The risks for complications during pregnancy, labour, delivery and puerperium were not increased. The occurrence of seizures during labour, delivery and early puerperium was significantly associated with discontinuation of AC. Eighteen per cent of our patients had increased seizure frequency during pregnancy which was not associated with decreased blood AC levels; however, a significant correlation with poor pre-pregnancy seizure control was found. Although there is an increased risk for $M J$ in the offspring of epileptic women, several approaches can be adopted in order to minimize risk factors. The importance of pre-pregnancy counselling and regular follow-up during pregnancy is emphasized.

\section{7.}

MRI Volumetric Study: Insights Into the Etiology of Temporal Lobe Epilepsy

F. CENDES, F. ANDERMANN, P. GLOOR, A. EVANS, T. PETERS, I. LOPES-CENDES, E. ANDERMANN and A. OLIVIER (Montreal, Quebec)

We studied 76 patients with intractable temporal lobe epilepsy (TLE), using MRI volumetric measurements of amygdala and hippocampus. We found significant atrophy of mesial structures, coinciding with the EEG focus, in $92 \%$ of those without foreign tissue lesions. The atrophy correlated with mesial temporal sclerosis (MTS) in the histopathology, and was more pronounced in patients with a history of prolonged febrile convulsion (PFC) in childhood.

Other pathology, such as gangliogliomata and neuronal migration disorders, was found in 10 patients with TLE without antecedent PFC. In 8 of these 10 patients, the amygdala and hippocampus were symmetrical and of normal volume.

There were 6 patients in whom no lesion nor definite volumetric asymmetry were found. Four of these 6 patients were operated and the histopathology showed mild MTS. These patients may represent one extreme of a spectrum of MTS in TLE, which is too mild to be reflected in the MRI. Another possibility for this lack of atrophy is nondiagnosed subtle neocortical epileptogenic lesions.

In conclusion, MRI volumetric atrophy of amygdala and hippocampus appeared to be specific for TLE. Volumetric studies generate numerical values allowing better comparison of the degree of mesial atrophy among subgroups of TLE, leading to better discrimination and understanding of this condition.

48.

\section{The Clinical Spectrum of Progressive Supranuclear Palsy}

\section{C.L. BARCLAY, A.E. LANG and J. DUFF (Ottawa; Toronto, Ontario)}

Progressive supranuclear palsy (PSP) is increasingly being recognized as having a much wider clinical spectrum than was originally appreciated. In order to document this spectrum we reviewed the records of all PSP patients seen at the Toronto Western Hospital Movement Disorders Clinic between 1983 and 
1992. A total of 79 patients met all necessary diagnostic criteria. The average age of onset was 62.9 (range $47-81$ ). The most common presenting symptoms were falls $(62 \%)$, gait difficulty $(55 \%)$ and speech problems (31\%). In spite of all patients having a supranuclear gaze palsy, only $29 \%$ had visual symptoms. Eight patients presented initially without a supranuclear gaze palsy and six of these had the syndrome of "pure akinesia". These individuals tended to have a more protracted course (duration to death or last follow-up 12 years versus 4.9 for the group as a whole). Abnormal involuntary movements (AIMS) were common with $45 \%$ having tremor (including $13 \%$ with tremor at rest), $27 \%$ with limb dystonia and $39 \%$ with lid opening disturbances (blepharospasm and/or apraxia of eyelid opening). Other AIMS (most often involving facial muscles) occurred in $10 \%$. Unusual symptoms included spontaneous arm elevations (3), breathing abnormalities (3), echolalia and palilalia (5) and moaning (2).

We conclude that involuntary movements including tremor and dystonia are common in PSP. In addition, a clinical variant exists characterized by pure akinesia with late onset supranuclear gaze palsy and this appears to confer a more benign course.

49.

Autonomic Dysfunction in a Family with Spinocerebellar Ataxia

J.D. STEWART, I. LOPES-CENDES and E. ANDERMANN (Montreal, Quebec)

Objective: To investigate the autonomic nervous system (ANS) in a family with a spinocerebellar ataxia (SCA).

Background: ANS dysfunction has been described as a characteristic feature of the multisystem degenerations. However, the involvement of the ANS in the inherited forms of SCA has rarely been reported.

Methods: We evaluated four patients from a large family presenting with an autosomal dominant form of SCA. Patients were assessed clinically, by neurophysiological and neuropathological evaluations, and by neuroimaging techniques. We tested cardiovascular autonomic reflexes by measuring beat-beat blood pressure and heart rate changes during deep breathing, lying to stand, and Valsalva manoeuvre.

Results: Two patients had a clear abnormality in heart rate control (parasympathetic cardiovagal function) but normal sympathetic vasoconstrictor function. These two patients had a more widespread central nervous system degeneration with signs of motor neuron disease and motor and sensory neuropathy. The two other patients with predominantly cerebellar abnormalities had normal autonomic cardiovascular tests.

Conclusions: Affected family members fall into two phenotypic groups, those with predominantly cerebellar abnormalities, and those with additional signs of motor system involvement and peripheral neuropathy. Parasympathetic autonomic dysfunction was found in patients with the latter phenotype only.
50.

Locus Heterogeneity in French-Canadian Families with Autosomal Dominant Spinocerebellar Ataxia

I. LOPES-CENDES, E. ATTIG, F. CENDES, M.I. BOTEZ and G.A. ROULEAU (Montreal, Quebec)

The spinocerebellar ataxia (SCAs) are a clinically heterogeneous group of neurological degenerative diseases. To date two loci have been mapped: ch 6p (SCA / locus) and ch 12q (SCA 2 locus). We have studied two large French-Canadian families segregating an autosomal dominant form of SCA. The $F C$ kindred with 27 individuals, 11 of whom are affected. Mean age of onset 39 years $(31-49)$. Symptoms are predominantly of cerebellar type, with ataxia of gait and limbs, dysarthria and dysmetria. The progression of symptoms is slow, compatible with a normal life span. The Gaspé kindred, one of the largest SCA pedigrees described, with 310 individuals, 54 of who are affected. Mean age of onset 45 years $(11-60)$. In this pedigree two diferent clinical patterns could be identified: onset in late 40 s, slow progression and predominantly cerebellar symptoms; and onset in early $20 \mathrm{~s}$, faster progression, marked multisystem involvement, including motor neuron disease and signs of peripheral neuropathy.

We performed linkage analysis in 90 individuals from both kindreds using anonymous DNA markers which flank the two previous described loci. We excluded linkage to ch $6 \mathrm{p}$ in both kindreds. In the $F C$ kindred linkage to ch $12 \mathrm{q}$ was also excluded. However in the Gaspé kindred linkage to $12 \mathrm{q}$ was confirmed.

Our results support previous findings of locus heterogeneity among SCAs. Furthermore we have shown evidence for a third locus, which is responsible for the disease in the $F C$ kindred.

51.

Endogeneous Affective Disorders in Essential
Blepharospasm

E. POURCHER, R.H. BOUCHARD, P. BARUCH, M.-J. FILTEAU and P.J. BÉDARD (Quebec, Quebec)

Essential blepharospasm is a focal form of dystonia with a higher prevalence in females and a latter age at onset than other forms of focal dystonias. Not unfrequently, these patients present with a depressive episode considered of a reactive nature. We compared the prevalence of mood disorders in 12 patients with essential blepharospasm (BS) to 12 patients affected by an equivalent esthetic and functional handicap (chronic unilateral hemifacial spasm) (HS) and submitted to the same treatment, botulinic toxin. Data concerning the index disease and personal and familial psychiatric history were gathered using a semistructured interview and Hamilton Depression and Anxiety Rating Scales. Comparisons were made using Student t-test or exact Fisher's test.

Results: BS and HS groups did not differ on age, disease duration, or prevalence of a current depressive episode. However, significant differences emerged for: (i) personal 
antecedents of major depression (BS $=10 / 12$ vs. $\mathrm{HS}=2 / 12 ; \mathrm{p}=$ $0.002)$. Bipolar disorder or pathological affective temperament according to Akiskal's classification (BS $=10 / 12$ vs. $\mathrm{HS}=5 / 12$; $\mathrm{p}=0.049$ ). Sub-optimal therapeutic response to Botox after serial injections ( $\mathrm{BS}=6 / 12$ vs. $\mathrm{HS}=0 / 12 ; \mathrm{p}=0.49$ ). Conversely, psychosocial stressors at disease onset were more frequent in HS patients ( $B S=2 / 12$ vs. $H S=8 / 12 ; p=0.0194$ ).

Conclusion: Rather than an association between reactive depression and BS, our results suggest the importance of genetic factors of mood disorders in the expression of an underlying lesion presenting as a blepharospasm.

52.

Autosomal Dominant Cerebellar Ataxia in a Large Saskatchewan Kindred: Clinical Features and Preliminary Genetics

R.K. MOSEWICH, J.R. DONAT, A. SCHUAIB and T. SIDDIQUE (Saskatoon, Saskatchewan; Chicago, Illinois)

A large Saskatchewan family with autosomal dominant cerebellar ataxia is reported.

A total of eight affected individuals ( 5 male, 3 female) were found from three generations. The mean age at onset was 40.8 years (range $=30-55$ ). The most prominent symptoms were slurred speech and unsteadiness.

Physical examination revealed mild cognitive impairment in most affected individuals. Optic atrophy was present in 4 patients but no patient had retinal degeneration. Prominent eye movement abnormalities were found which included saccadic dysmetria, horizontal and occasionally vertical gaze evoked nystagmus, jerky smooth pursuit and gaze apraxia. An ataxic, scanning dysarthria was noted in all patients. Motor examination and reflexes were normal in all cases. Vibratory and position sensation was normal in all except 3 patients. Abnormalities in finger to nose testing, heel to shin testing and gait were prominent.

The results of clinical investigations as well as the preliminary results of linkage analysis will be presented.

53.

Defective Coordination Between Postural Mechanisms for Maintaining Equilibrium and Voluntary Movement in Parkinson's Disease

R.G. LEE, F. VIALLET, I. TONOLLI, R. AURENTY and J. MASSION (Calgary, Alberta; Marseille, France)

Many voluntary movements produce displacement of the body center of gravity and postural adjustments are required before or during the movement to preserve normal equilibrium. We studied anticipatory postural adjustments in 5 parkinsonian patients and 4 controls during a lateral leg raising task while standing on a free platform. Kinematics were monitored with an ELITE motion analysis system using reflective markers at multiple sites on the trunk and legs, and surface EMG activity was recorded from 4 muscles in each leg. In normal subjects, prior to displacement of the moving leg, there is a shift of the center of pressure on the force platform initially toward the moving leg and then a transfer of weight to the support leg. These force changes are accompanied by displacement of the trunk markers toward the support side. In the parkinsonian patients the following abnormalities were observed: (i) the amplitude of the initial change in the center of foot pressure was diminished, (ii) the interval between the initial force change and displacement of the moving leg was prolonged, and (iii) the amplitude and velocity of displacement of the trunk markers toward the support side was reduced. These results suggest that mechanisms for programming postural adjustments are impaired in some parkinsonian patients.

54.

Clobazam in Refractory Essential Tremor

C.L. BARCLAY and A.E. LANG (Ottawa, Ontario; Toronto, Ontario)

Essential tremor (ET) remains a major source of disability in many patients despite therapy with beta-blockers and primidone. Alcohol responsivity suggests that GABA plays an important pathophysiological role and benzodiazepines also may reduce tremor, however, side effects often limit their usefulness. Clobazam is reportedly better tolerated than other benzodiazepines and so we examined its efficacy in 10 patients with refractory ET. Patients ranged in age from 39 to 88 (mean 72.8) and had failed treatment with a mean of 3.8 medications either due to intolerable side effects or lack of benefit. Clobazam was begun at $10 \mathrm{mg}$ per day and increased by $5 \mathrm{mg}$ per week up to $40 \mathrm{mg}$ per day or lower if marked benefit or intolerable side effects occurred. Open-rating assessed functional disability and severity of tremor while a blinded examiner rated samples of writing and drawing. Three patients obtained a useful symptomatic and clinically demonstrable improvement, 1 worsened, 5 developed intolerable side effects and 1 died of unrelated cardiovascular causes during the trial. We conclude that clobazam is beneficial in some patients with refractory ET. Side effects may limit its use, however, our patient population was elderly and had already demonstrated a sensitivity to medications. Further study is required to clarify the role of clobazam in ET.

\section{5.}

\section{The Clinical Value of SPECT in Stroke}

A.V. ALEXANDROV, L.E. EHRLICH, L.T. SMURAWSKA, A. PIRISI, C.F. BLADIN, C.B. CALDWELL, S.E. BLACK and J.W. NORRIS (Toronto, Ontario)

SPECT is widely used for management of stroke patients but its value is controversial. We therefore decided to test HMPAOSPECT in acute stroke patients and evaluate its additional value to clinical examination and CT-scanning.

Two hundred fifty consecutive stroke patients were studied (120 males and 130 females, mean age $74 \pm 12 \mathrm{yrs}$ ). Twenty- 
one $(8.4 \%)$ had cerebral hemorrhage and $229(91.6 \%)$ had ischemic stroke: $98(39 \%)$ - cardioembolic, $16(6 \%)$ - symptomatic carotid disease, $23(9 \%)$ - lacunar stroke, and 92 $(38 \%)$ were of unrevealed cause ("cryptogenic"). Two hundred fifty SPECT studies were performed on the fifth day and 113 at two weeks after the onset. Severe perfusion deficits were associated with the largest volume of CT-lesions $(p<0.0003)$ and with the poorest short-term outcome (Canadian Neurological Scale) $(p=0.03)$. Hyperperfusion was common in cardioembolic stroke $(36 \%)$ compared to the rest $(17 \%)(p=0.0001)$. Normal perfusion was seen in $50 \%$ of lacunes $(p<0.00001)$. SPECT alone did not differentiate cerebral hemorrhage from hemorrhagic infarction and infarction due to occlusion of intracranial artery. Moreover, chronic infarction with cystic change can produce the same focal absence of perfusion.

SPECT is an ancillary investigation to CT but is of particular value when CT is negative. Normal flow suggests lacunar stroke, while hyperperfusion points to an embolic event (if there is no significant carotid artery disease, it is more likely to be a cardioembolic stroke). Patients with severe perfusion deficits, irrespective of CT or clinical findings, all fared badly.

\section{F. NEUROSURGICAL ONCOLOGY}

\section{6.}

\section{BUdr Radiosensitization in a Rate C-6 Glioma Model}

\section{R.W. GRIEBEL, A. AGRANOVICH and L. TAN (Saskatoon,} Saskatchewan)

The radiosensitization properties of BUdr (5-bromo-2' deoxyuridine) have been well established. Clinical application of the drug has been limited by systemic side effects from intravenous and intraarterial infusions. We have attempted to establish a reproducible animal model to further study alternate delivery routes of the drug.

Five animal treatment groups have been studied. Group A, the control group, receive tumors but no treatment. Group B animals receive cranial radiation only $(1,000 \mathrm{cGy}$ in $5 \mathrm{frac}-$ tions). Groups C, D and E animals were treated with BUdr in addition to the radiation. Group $\mathrm{C}$ animals receive the drug intraperitonealy $(83 \mathrm{mg} / \mathrm{kg})$. Group $\mathrm{C}$ and Group E animals underwent intracranial infusion of the drug via an implanted canula.

Outcome measures of weight loss, neurological symptoms, tumor presence and symptom free days were analyzed. The addition of BUdr demonstrated a significant advantage in tumor cure rates $49 \%$ vs. $80 \%$ ( $p=0.006$ ) over radiation alone. No significant improvement could be demonstrated between the various modalities of drug delivery.

Radiolabelling for the drug demonstrated intra-tumor distribution of the drug was unaffected by delivery modality.
57.

\section{Experimental Treatment of Glioma with TK(-) HSV-1 Virus}

W.W.-G. JIA, M. McDERMOTT, F. TUFARO and J. GOLDIE (San Francisco, U.S.A.; Vancouver, British Columbia)

Thymidine kinase deficient type 1 herpes simplex virus (tk(-) HSV-1) is severely impaired in replication in postmitotic cells but replicates normally in dividing cells causing cell death. In the present study, we used rat 9L glioma cells and brain tumor model to test the effectiveness of tk(-) HSV-l in brain tumor treatment. In vitro, the tk(-)HSV-1 virus specifically and rapidly destroyed $100 \%$ of $9 \mathrm{~L}$ glioma cells but not primary cultured cortical neurons. Trisodium phosphono formate (PFA), an anti-viral drug independent of tk activity, completely inhibited viral replication at a concentration of $100 \mu \mathrm{g} / \mathrm{ml}$. 9L cells were then implanted intracranially in rats and either the virus or saline was injected into the same site two weeks later. In the control group, $100 \%$ of rats with tumors died within 22 days post-implantation. In contrast, only $20 \%$ of the animals treated with $10^{5} \mathrm{PFU}$ viral particles died in the same period of time and the mortality tends to further decrease with increases of concentration of virus. All rats intracortically injected only with virus survived our longest period of follow-up (4 months) without apparent ill effect, suggesting the virus itself (up to $10^{9} \mathrm{PFU}$ ) is not lethal. Histological examination showed severe necrosis in the tumor at the site of viral injection. In some animals treated with higher titers of virus $\left(10^{8} \mathrm{PFU}\right)$, the tumors disappeared completely. These results strongly suggest that tk(-) HSV-1 mutants can selectively destroy glioma in both in vitro and in vivo and as such are promising candidates for therapeutic use in patients with malignant gliomas.

\section{8.}

\section{Expression of Timp-1 and Timp-2 by Human Malignant Astrocytomas}

\section{J.T. RUTKA, S.L. SMITH, K. FUKUYAMA and A. HAUETT (Toronto, Ontario)}

Human malignant astrocytomas are highly invasive neoplasms which infiltrate diffusely into regions of normal brain. Tumor cell invasion is a complex cellular process mediated in part by the elaboration of tumor-associated proteolytic enzymes and by various inhibitors of metalloproteinases. We have previously shown that human astrocytomas in vitro secrete collagenase type IV, gelatinase, and tissue inhibitors of metalloproteinases (TIMPs) TIMP-1 and TIMP-2 (Cancer Res 1990: 50; 2322). In the present study we sought to determine the mRNA expression of TIMP-1 and TIMP-2 in human astrocytoma cell lines and a variety of brain tumor specimens by Northern and in situ hybridization analyses. In addition, we attempted to correlate TIMP-1 and TIMP-2 expression with astrocytoma invasion in an in vitro invasion model system. TIMP-1 and TIMP-2 were variably expressed in all malignant astrocytoma cell lines. Interestingly, among brain tumor specimens, there was a 
tendency for the more differentiated, less anaplastic tumors to express higher mRNA levels of TIMP-1 and TIMP-2. In situ hybridization analyses showed specific intratumoral intracytoplasmic localization of mRNA for TIMP-1 and TIMP-2. Using the in vitro model system for invasion, we found variability in radio-labelled astrocytoma cell invasion across matrigel-coated filters. Those astrocytoma cell lines with reduced TIMP-1 and TIMP-2 mRNA expression invaded more readily through the matrigel membrane than did astrocytoma cell lines with abundant mRNA for these inhibitors of metalloproteinases.

\section{9.}

\section{Management and Outcome of Low-Grade Astrocytomas of the Midline in Children: A Retrospective Review}

\section{H.J. HOFFMAN (Toronto, Ontario)}

Low-grade astrocytomas of the midline of the brain can be difficult to manage because of their location. To evaluate treatment and outcome, we carried out a retrospective study of children with midline low-grade astrocytomas admitted to The Hospital for Sick Children from 1976 to 1991. Eighty-eight children with biopsy-proven low-grade astrocytomas were identified. Forty-three tumors occurred in the optic pathways or hyptoahalamus, 13 in the thalamus, 7 in the pineal region, 14 in the midbrain, and 11 in the medulla. Patient follow-up ranged from 6 months to 15 years with a mean of 4 years, 9 months. Overall outcome was related to extent of resection, histologic type and location. Partial resections were often associated with involution of tumor. Response to radiation was variable with serious sequelae seen. Thirty-three patients had recurrence, often with good response to reoperation, but 12 of these patients died. The probability of survival was calculated to be $96 \%$ at 1 year, $91 \%$ at 5 , and $80 \%$ at 10 years. Our study suggests that resection should be considered in all patients both at presentation and recurrence.

60.

\section{The Surgical Morbidity of Aggressive Resection of Posterior} Fossa Tumors in Childhood

\section{COCHRANE, B. GUSTAVSON, K. POSKITT, P. STEINBOK} and J. KESTLE (Vancouver, British Columbia)

The morbidity associated with gross total removal (GTR) of posterior fossa (PF) tumors in children is well recognized although it is rarely isolated from other factors that comprise management morbidity.

This study reviews complications that were attributed to operation or occurred in the immediate post-operative period in 103 patients undergoing gross total removal of their PF tumor between 1982 and 1992 .

Gross total resection was accomplished in 101 patients. Two patients required repeat craniotomy for missed tumor. Complications included intra-operative complications (6), surgical site hemorrhage requiring craniotomy (3), gastrointestinal hemorrhage (2), hydrocephalus required shunt (8), wound infection and pseudomeningocele (10), and spinal deformity (6).
Worsening of pre-operative deficit (new cranial nerve palsies, worsening ataxia, bulbar dysfunction including apnea and mutism and seizures) occurred in 53\% of PNET (18/34), $56 \%$ of ependymomas $(10 / 19)$, and $22 \%$ of astrocytomas (11/50). Recovery of post-operative deficit occurred in $11 \%$ of PNET (2/18), 50\% of ependymoma (5/10) and $64 \%$ of astrocytoma $(7 / 11)$. Recovery of cranial nerve, cerebellar and bulbar function occurred within 6 months.

Some complications are potentially avoidable. The occurrence of post-operative neurological disability remains a function of the type, location and invasive character of the tumor.

61.

\section{Medulloblastoma in Adults - the Montreal Neurological Hospital Experience}

M. VASSILYADI, J.G. VILLEMURE, J.P. BAHARY and K. VILLEMURE (Montreal, Quebec)

Between 1950 and 1992 there were 27 cases of medulloblastoma, occurring between the ages of 16 and 59. Thirteen were males and 14 females. The most common symptom (96\%) and chief complaint was headache (mostly fronto-occipital). The length of symptoms before presentation ranged between 0.5 and 24 months. Only $67 \%$ demonstrated papilloedema.

Five patients died within ten days of admission and nine were lost to follow-up. Four patients died between 1.7 and 4.7 years after presentation. Nine patients are alive today (the longest since 1978). In these nine, surgery included seven complete resections via a suboccipital craniectomy, one subtotal, and one biopsy. All received megavoltage irradiation ranging between $5600-6200$ cGy to the posterior fossa and $3000-3500$ cGy to the entire spine over a six week period.

Desmoplasia was associated with a favorable prognosis; hemispheric tumors exhibited desmoplasia more often than midline tumors $(p<0.05)$. There was no relationship between tumor site and patient age. Survival rates after one, five, and ten years were $100 \%, 33 \%$, and $20 \%$ respectively. A relationship was found between time of relapse and death $(R=0.96)$. The rate of metastasis was found to be $33 \%$.

62.

Predictors of Outcome from Surgery for Benign Tumors of the Cavernous Sinus

M.D. CUSIMANO, L.N. SEKHAR, S. POMONIS, C.N. SEN and W. HIRSCH (Toronto, Ontario; Pittsburgh, New York, U.S.A.; Athens, Greece)

A retrospective review of the 125 patients (avg age 50) with benign tumors of the cavernous sinus (CS) who had surgery upon the CS from 1983-1992 was performed. Average followup was 29 months.

A total or near total resection was achieved in $80 \%$ and was more likely in patients with the "Non-Permeative" tumors: angiofibromas, neurilemmomas, chondroblastomas, epider- 
moids and hemangiomas. Although the majority of patients suffered minor or treatable complications, six patients remained disabled, four as a result of infarctions and two due to meningitis. Pre-operative radiotherapy was a predictor of major complications.

Of the patients who entered surgery with excellent/good binocular vision, $60 \%$ remained stable or improved. Overall post-operative Karnofsky scores were not significantly different from pre-operative scores although those entering surgery with higher scores had the best chance for good outcomes post-operatively.

There was no perioperative deaths. Two patients died of the delayed sequelae of meningitis, five due to tumor growth and five unrelated to the surgery or tumors. Further follow-up will be necessary before a definitive statement can be made regarding recurrence.

63.

Epidural Spinal Metastases from Malignant Melanoma: Demographics, Prognosis, and Treatment

\section{J.H. SAMPSON, J.H. CARTER, A.H. FRIEDMAN and H.F. SEIGLER (Durham, U.S.A.)}

Metastatic melanoma is generally thought to be radioresistant and symptomatic spinal metastases from melanoma are rare. Consequently, the demographics, risk factors, role of irradiation and surgery, and prognosis for such patients remains unknown.

From 1970 to $1990,6,953$ patients with melanoma were seen at our institution. A retrospective review identified 39 patients with neurologic sequelae from spinal metastases. These patients had a mean age of $49.7 \pm 12.7$ years and 19 were female. The mean time interval from diagnosis of primary melanoma to spinal metastases was 3.9 years. Pain or myelopathy were equally frequent presentations and effective diagnosis was made in most cases by myelogram alone.

Risk factors associated with spinal metastases induced ulcerated primary lesions $(p=0.040)$, uncommon histologies $(p=$ $0.033)$, and a greater number of other metastatic sites $(p<0.01)$. Sixty-four per cent of patients (25/39) had at least one other site of distant metastasis when diagnosed with a spinal metastasis.

Seventy per cent of patients were improved by therapy, but contingency table analysis demonstrated no difference in neurological recovery when irradiation, decompressive laminectomy, or both were employed $(p>0.74)$. Actuarial life table analysis also demonstrated no increase in survival with any therapy relative to controls that received no local therapy $(p>0.10)$. The median survival time for all patients with spinal disease was only 54 days.

In conclusion, although a few patients are dramatically improved and survive for long periods after treatment for symptomatic spinal metastases from metastatic melanoma, this diesease is usually associated with disseminated metastases and a short survival time which is unaffected by local therapy.
64.

\section{Fractionated Stereotactic Radiotherapy of Small Benign Axial Tumors in the Adult - Report of 10 Cases}

\author{
J.-G. VILLEMURE, L. SOUHAMI, R. LEBLANC, A. OLIVIER, \\ R. POKRUPA, J.P. BAHARY and E.B. PODGORSAK \\ (Montreal, Quebec)
}

Ten patients with symptomatic axial tumor were treated with fractionated stereotactic irradiation. The lesions were located in the posterior 3rd ventricle, midbrain, pons and medulla; histologically, there was 1 pineocytoma, 1 papilloma, 2 subependymomas and 6 astrocytomas; tumor volume varied from 10 to 25 $\mathrm{mm}$. There were 5 males and 5 females, and the age ranged from 16 to 73-years-old (median 32). Treatments were carried out using the dynamic radiosurgical technique with a $10 \mathrm{MV}$ linear accelerator. The fractionated regimen consisted of $42 \mathrm{~Gy}$ given in 6 fractions of 7 Gy at the $90 \%$ isodose surface which corresponded to the periphery of the lesion with a margin of a few mms. Eight patients showed a radiological response. Most patients with cranial nerve involvement showed early clinical improvement. Treatments were well tolerated and there were no acute complications. Two patients showed tumor progression after early response. These data suggest that fractionated stereotactic irradiation of small axial tumor is a relatively safe treatment and appears beneficial in some selected lesions.

\section{5.}

\section{Frameless Stereotaxy for Radiosurgical Follow-Up}

M.L. SCHWARTZ, R. RAMASESSAN, C.S. YOUNG, P.F. O'BRIEN, P. HUDOBA and P. DAVEY (Toronto, Ontario)

In our centre, more than 80 patients have been treated with linear accelerator stereotactic radiosurgery. Angiographic, CT and MRI images are generated and the target coordinates calculated in three dimensions. For CT scanning, cross sections of perpendicular and oblique fiducial markers are seen.

For follow-up CT scans done without the frame, a virtual frame is generated by means of a computer program that placed fiducial markers on each CT scan cut, as if the patient had been wearing the OBT frame and the scan produced with the gantry parallel to the base of the frame. The position of the oblique marker may be calculated by knowing the thickness and position of each CT cut. Various natural fiducial markers such as the anterior clinoid process and the internal auditory meatus are identified by coordinates in the scan with the patient wearing the real frame and in the scan with the virtual frame applied. A transformation matrix is utilized to establish the equivalence between the original CT scan with the real frame applied and subsequent scans without the real frame but with the virtual frame applied. In effect, the virtual frame is re-applied in exactly the same position as the real frame.

Lesion measurements may then be duplicated and growth or regression accurately established. The uncertainty in this system of re-application resides in possible patient movement, CT scan 
slice thickness and inter-observer error in the identification of natural fiducial markers. This system is particularly suitable for acoustic neuroma follow-up because thin $\mathrm{CT}$ slices of the natural fiducial markers are produced.

66.

\section{The Use of Fractionated Stereotactic Radiotherapy in Intracranial Meningiomas}

J.A. ESPINOSA, J.-G. VILLEMURE, L. SOUHAMI, J.-L. CARON, A. OLIVIER, J.-P. BAHARY and E.B. PODGORSAK (Montreal, Quebec)

From June 1989 to February 1992, 16 patients with surgically inaccessible meningiomas underwent fractionated stereotactic radiotherapy. There were 11 females and 5 males with median age of 53 years.

Treatments were delivered using the dynamic rotation radiosurgical technique which employs a $10 \mathrm{Mv}$ linear accelerator. Total dose was $42 \mathrm{~Gy}$, fractionated on 6 sessions given on alternate days for 2 weeks. The dose was prescribed at the $90 \%$ isodose surface which corresponded to the periphery of the tumor plus a few millimetres in margin. The stereotactic frame was used in the first session. For subsequent treatments, the head was immobilized with a halo ring affixed to the table.

At a median follow-up time of 15 months (range: 9 - 40), 7 patients have shown a favorable radiological response (4 with tumor reduction and 3 with central necrosis), whereas in other 7 there were no changes. Radiologic evidence of tumor growth was clear in 2 cases.

Treatments were well tolerated. However, serious late complications developed in 2 patients.

These preliminary data suggest that fractionated stereotactic radiotherapy is a feasible and effective treatment for selected patients with meningiomas in areas of difficult surgical access.

\section{G. CLINICAL NEUROPHYSIOLOGY}

67.

EEG Findings and Surgical Outcome of Frontal Lobe Epilepsy in Patients Investigated with Depth Electroenchephalography

L.F. QUESNEY, F. CENDES, A. OLIVIER, F. DUBEAU and F. ANDERMANN (Montreal, Quebec)

We studied the interictal and ictal findings in 18 patients with frontal lobe epilepsy (FLE) investigated with depth electrodes (SEEG) implanted bilaterally in the orbitofrontal, cingular and superior frontal regions, as well as in both temporal lobes.

Extracranial EEG findings: only 7 (39\%) patients presented focal/regional interictal frontal lobe spiking. Four patients showed bifrontal spiking, and 4 had interictal abnormalities over both temporal lobes. The remaining 3 patients exhibited no interictal spiking. Ictal recordings in these patients provided no seizure onset localization.

SEEG findings: focal/regional ictal EEG onsets were documented in $10(56 \%)$ patients. The extracranial recordings in these 10 patients showed focal/regional spikes in 6 ; no interictal spikes in 2; and bitemporal epileptic discharges in 2. A structural lesion in the frontal lobe was demonstrated in these 2 patients.

Ictal SEEG onset was bifrontal in 6 and fronto-temporal in the remaining 2 patients.

Surgical outcome in 15 patients with more than 2 years post-operative follow-up (mean 7.8, range 2 - 15 years) was: $67 \%$ class I or II, and $33 \%$ class II or IV (Engel's classification). Factors related with good prognosis were unilateral frontal lobe spiking on extracranial EEG, and presence of a structural lesion $(\mathrm{p}<0.05$, chi-square $)$.

68.

\section{Epileptiform Bursting Produces Prolonged NMDA- Dependent Hippocampal Hyperexcitability}

\section{J.H. SCHNEIDERMAN (Toronto, Ontario)}

Partial blockade of GABA ${ }_{A}$-mediated inhibition by low concentrations of penicillin $(<0.85 \mathrm{mM})$ produced low frequency $(2-4 \mathrm{~Hz})$ "pro-convulsant" oscillations in hippocampal slices which also precede spontaneous bursting in high concentrations (3.4 $\mathrm{mM}$ ) and appear similar to EEG rhythms recorded in some patients at seizure onset. Normal spontaneous activity recovered less than 30 minutes after penicillin rinse provided synchronized bursting was avoided. However, prolonged hyperexcitability followed epileptiform bursts. Burst amplitude declined more slowly than anticipated during convulsant washout. "Postburst" oscillations with different frequency content than proconvulsant oscillations persisted for at least 4 hours and were associated with marked potentiation of excitatory synaptic responses.

Spontaneous bursts occurred in $0.85 \mathrm{mM}$ penicillin only after threshold stimuli selectively potentiated NMDA-dependent EPSPs which then triggered one or more bursts. NMDA antagonists (APV) blocked this response and prevented bursts and long-lasting hyperexcitability. In slices pre-treated with APV, $3.4 \mathrm{mM}$ penicillin produced large, long interval bursts but these disappeared promptly upon penicillin washout and were not followed by post-burst oscillations.

Epileptiform bursts can produce prolonged hippocampal network hyperexcitability which may increase susceptibility to further seizures. NMDA receptors are required to establish these plastic changes but not to maintain them. 
69.

Seizures Induced by Cholinergic Stimulation of the Basal Forebrain and Diencephalon

\section{R.S. MCLACHLAN, S. BRUDZYNSKI and J. CRUICK- SHANK (London, Ontario)}

Acetylcholinesterase inhibitors, acetylcholine, or acetylcholine analogues are potent epileptogenic agents while anticholinergic agents can prevent seizures initiated by cholinomimetics. Stimulation of basal forebrain and diencephalic regions with carbachol results in generalized seizures. However, it is not yet clear which regions within these subdivision are most sensitive to cholinomimetics. The goal of this study was to map the epileptogenic effects of carbachol when injected into different sites of the basal forebrain and diencephalon of the rat brain.

Seizures were assessed behaviorally on a five-stage scale with electroencephalographic controls. Direct intracerebral injections of high dose carbachol $(3 \mu \mathrm{g})$ induced motor seizures from $40 \%$ of all injected brain sites. Injections of low dose carbachol $(1 \mu \mathrm{g})$ or isotonic saline into the same brain sites did not cause any behavioral or electrographic seizures. While injection sites producing minor motor seizures (stage $1-4$ ) and wet dog shakes were scattered throughout the brain regions studied, the majority of sites $(84 \%)$ producing generalized seizures (stage 5) were concentrated in or around the zona incerta and the subthalamus. Spearman's correlation test revealed a significant correlation between frontal, or horizontal stereotaxic coordinates, and the severity of seizures pointing at zona incerta as the most sensitive to carbachol in inducing generalized seizures. The possibility that zona incerta may be involved in the pathophysiology of generalized epilepsy should be further explored.

70.

REEGAL (Retrospective EEG AnaLysis): A New Clinical Tool for the Quantification of Paper-Based EEGs

\section{M.J. McKEOWN and G.B. YOUNG (London, Ontario)}

The quantification of the EEG permits a more objective comparison between records than is possible with visually interpreted raw tracings. This is very useful in a number of clinical situations with respect to diagnosis, treatment and prognosis. Unfortunately, a wealth of clinical EEG information is presently stored as paper traces which have, in the past, been difficult to quantitate. We describe the development of a clinical tool, REEGAL (Retrospective EEG AnaLysis), which is an inexpensive, easy to use, easily implemented tool which permits the quantification and spectral analysis of such paper-based EEG records. Also, a sister software package has been developed which enables the computation or correlation (coherence) and phase analysis from multiple epochs that have been quantified by REEGAL.

The processing techniques required to extract the quantified information from paper-based EEGs are described. As a demon- stration, typical raw EEG traces are shown in conjunction with their computed power spectra.

71.

Acute Myopathy of Intensive Care Unit Patients: Serial Electrophysiological Studies

D.W. ZOCHODNE, D.A. RAMSAY, V. SALY and R. SEMMLER
(Calgary, Alberta; London; Kingston, Ontario)

Recent reports have identified several caes of quadriplegic myopathy associated with the use of nondepolarizing muscle blocking agents (NDMBAs) and glucocorticoids. We report electrophysiological findings in seven intensive care unit patients who developed respiratory and limb muscle weakness with evidence of an acute myopathy in association with NDMBAs.

Several features were identified: (i) a neuromuscular transmission deficit that persisted for up to 7 days following withdrawal of vecuronium; (ii) low amplitude motor $\mathrm{M}$ potentials, abnormal spontaneous activity on needle elctromyography and relatively preserved sensory conduction; (iii) not all patients had received steroids or were asthmatic; (iv) two patients given vecuronium had very high serum $\mathrm{CK}$ levels and developed acute renal failure; myoglobinuria was identified in one of these patients and suspected in the second; our previous histologic studies have identified widespread muscle fiber necrosis; (v) rises in motor $\mathrm{M}$ potentials accompanied clinical recovery, and (vi) one patient with an acute myopathy later developed polyneuropathy during complications of sepsis and multiple organ failure.

This apparent iatrogenic complication of intensive care unit treatment may be severe, but is reversible and possibly avoidable. Our findings implicate NDMBAs in its development. Electrophysiological studies provide important prognostic guidance.

72.

A Comparison of Local Nerve and Dorsal Root Ganglion Blood Flow in Experimental STZ-Induced Diabetic Neuropathy of Rats

D.W. ZOCHODNE, L.T. HO and J. RACICOT (Calgary, Alberta; Kingston, Ontario)

In human diabetes, sensory abnormalities are usually more prominent than motor changes. Widespread endoneurial ischemia would not explain this selectivity of sensory fibers, because most nerve trunks include motor and sensory axons.

We examined local blood flow in the sciatic nerve and L4 dorsal root ganglia (DRG) of Sprague-Dawley rats 4 months after the intraperitoneal injection of streptozotocin (STZ) or citrate buffer alone. Animals were accepted as diabetic if the blood glucose 3 - 5 days after STZ was greater than or equal to 16.0 $\mathrm{mmol} / \mathrm{L}$. Serial measurements of caudal motor and caudal sensory conduction monitored the progress of neuropathy and at 
endpoint, in vitro conduction was studied in resected sural nerve. Local blood flow was extrapolated from hydrogen clearance curves recorded from the sciatic nerve endoneurium or DRG.

Diabetic animals gained less weight than controls and had cataracts. Multifiber nerve recordings identified slowing of caudal and sural sensory conduction and more mild changes in caudal motor conduction. As in our previous work, sciatic endoneurial blood flow measurements were similar between diabetic and nondiabetic animals. In the DRG, there was a trend ( $p=0.03$; one tailed test) toward lower blood flow in diabetic animals $(35.8 \pm 4.6 \mathrm{ml} / 100 \mathrm{~g} / \mathrm{min}$ in controls vs. $24.5 \pm 3.1$ $\mathrm{ml} / 100 \mathrm{~g} / \mathrm{min}$ in diabetics).

Selective DRG involvement by diabetic microangiopathy could account for prominent sensory involvement in diabetes but further work is required to support this hypothesis.

73.

\section{Carpal Tunnel Syndrome: Topography of the Sensory} Abnormalities in 100 Hands

L. DURCAN, G. RIESS and J. STEWART (Montreal, Quebec; Ann Arbor, U.S.A.)

Objective: To evaluate the variable patterns of sensory loss in patients with carpal tunnel syndrome (CTS).

Methods: In this prospective study, patients were included if they had both symptoms of CTS and confirmatory nerve conduction studies. A sensory examination of the palmar surfaces of fingers and hand was done with pin and a bristle, and sensory abnormalities mapped.

Results: $75 / 100$ hands had abnormalities to pin or bristle or both. Of these 75 only 7 had complete median sensory loss. The other 58 were partial, in the following patterns: distal, all digits - 13; distal, some digits -26 ; one web space -7 ; one side digit, all digits -4 ; one side digit, some digits -18 . Pinprick loss only was found in 12/75 patients; light touch loss only in $14 / 75$; both in $49 / 75$.

Conclusion: In CTS, partial sensory loss is more common than complete. Patterns of sensory loss suggest that compression of the median nerve can selectively damage: (i) longest fibers, (ii) specific fascicles, and (iii) specific types of sensory nerve fibers.

\section{4.}

\section{Neurophysiological Assessment of Failure to Wean From a Ventilator}

J. MAHER, F. RUTLEDGE, H. REMTULLA, A. PARKES, L. BERNARDI and C.F. BOLTON (London, Ontario)

Background: Electrophysiological assessment of patients who fail to wean from a ventilator has received limited attention despite such patients being at risk of significant morbidity and mortality.

Methods: We determined amongst these patients: (i) their spectrum of neuromuscular disorders, and (ii) the association between neuromuscular findings in their limbs and their respiratory system.

Results: Thirty-eight to 40 persons $(95 \%)$ had a neuromuscular disorder detected, 15 of whom each had 2 or 3 such disorders. Thirty-three had a polyneuropathy, 10 impaired central respiratory drive, 5 a phrenic nerve palsy, 5 a myopathy and 3 impaired neuromuscular junction transmission. Of those with a polyneuropathy, $45 \%$ had normal respiratory studies. Also, despite $95 \%$ have a naueomuscular disorder, only $65 \%$ had electrophysiological evidence of respiratory involvement.

Conclusions: One or more neuromuscular disorders were almost always present in patients who failed to wean from mechanical ventilation. However, electrophysiological assessment of the respiratory system was frequently normal, despite the presence of a neuromuscular disorder of the limbs.

75.

Effects of Induced Hypothermia on MEPs and EMG Interference Patterns in Patients with Chronic Spinal Cord Injury

K.C. HAYES, P.J. POTTER, J.T.C. HSIEH, D.L. WOLFE, G.A. DELANEY and A.R. BLIGHT (London, Ontario; Chapel Hill, U.S.A.)

We tested the hypothesis that reduction in core temperature would enhance central motor conduction in spinal cord injured (SCI) patients with compressive or contusive type lesions and conduction deficits due to demyelination. Whole body hypothermia was induced by controlled circulation of a coolant through a "microclimate" head and vest garment while reductions in oral $\left(-1^{\circ} \mathrm{C}\right)$ and limb temperature $\left(-3.5^{\circ} \mathrm{C}\right)$ were monitored. Fourteen $(n=14)$ patients and eight $(n=8)$ healthy adult controls served as subjects. Maximal voluntary EMG interference patterns were recorded from nine of the SCI patients. Seven of these patients showed a cooling induced increase in voluntary motor unit activity. Motor evoked potentials (MEPs) were recorded from ten of the patients following transcranial magnetic stimulation of motor cortex. Cooling resulted in an increase in MEP amplitude in six of the patients. This increase was sustained or increased on rewarming in four of the patients. The results support the hypothesis that cooling ameliorates motor conduction deficits in certain types of SCl patients and fit with the emerging view that central demyelination constitutes an important part of the pathophysiology of motor dysfunction in SCI patients.

76.

Percutaneous Electrical Stimulation at the Skull Base in Humans

D.A. HOULDEN, M.L. SCHWARTZ, C.H. TATOR and P. ASHBY (North York; Toronto, Ontario)

Electrical stimulation over the skull base can evoke muscle responses from the arms and legs. Previous investigators 
(Ugawa et al. 1991) concluded that the majority of these evoked muscle responses are mediated by the corticospinal tract. We investigated the pattern of muscle activation following percutaneous electrical stimulation of the skull base using a Digitimer D180 stimulator in 9 normal subjects. Electromyographic recordings were obtained from triceps, biceps, first dorsal interosseous (FDI), erector spinae, abdominal wall muscles, vastus medialis, tibialis anterior and soleus at varying stimulus intensities. Low stimulus intensities recruited triceps (a muscle with weak corticospinal facilitation, Phillips and Porter 1964) before FDI (a muscle with strong corticospinal facilitation) in all subjects. The early recruitment of triceps suggests that low intensity skull base stimulation may preferentially stimulate motor pathways other than the corticospinal tract thereby offering a technique for investigating and monitoring multiple motor pathways. High stimulus intensities evoked responses from all muscles. The estimated percentage of the total alpha motoneuron pool being recruited for each muscle was higher for the upper limb than the lower limb at all stimulus intensities. Spinal cord conduction velocity was $65 \mathrm{~m} / \mathrm{s}$ and $68 \mathrm{~m} / \mathrm{s}$ in fastest conducting central motor pathways to upper and lower limb motoneurons respectively.

77.

\section{Development and Validation of a Quantitative Measure of} Somatosensory Evoked Potentials

\section{R. MOULTON, S. KONASIEWICZ and P. O'CONNOR (Toronto, Ontario)}

This paper describes the development and testing of a computer algorithm to automate the identification of somatosensory evoked potential (SSEP) peaks and quantify the grading of SSEPs. The algorithm iteratively identifies peaks in the SSEP over progressively shorter segments of the SSEP. Filtering of noise is based on slope, amplitude and frequency characteristics of peaks.

We tested the accuracy of computerized peak detection and evaluated grading schema using a test set of 60 SSEPs. The test SSEPs were ranked from best to worst by the programmer (RJM), a blinded expert (PO), and by various computer grading schema.

The computer algorithm recognized $95 \%$ of peaks identified by visual inspection. Twelve per cent of peaks identified by the computer were noise. Summed peak to peak amplitude yielded the most accurate ranking of SSEPs. Rank correlation between computer and visual grading was $r=0.82$ for $\mathrm{PO}, \mathrm{r}=0.92$ for RJM, p $<0.0001$ for both. Computer and manually summed amplitudes were highly correlated (Pearson $r=0.98, p<0.0001$ ). Correlation between the 2 expert graders was $0.86, p<0.0001$.

Automatic grading of SSEPs using summed peak to peak amplitude is highly correlated with expert grading. The measure is objective, continuous, and well suited to statistical analysis.

\section{H. CEREBRAL ISCHEMIA}

78.

\section{Voltage Sensitive Calcium Channel Activation and Brain pH in Focal Cerebral Ischemia}

\section{M.J. HOGAN, S. OSUGA and A.M. HAKIM (Ottawa, Ontario)}

Calcium channel activation, assessed by in vivo $\left[{ }^{3} \mathrm{H}\right]$ nimodipine binding, and tissue $\mathrm{pH}$, measured using $\left[{ }^{14} \mathrm{C}\right] \mathrm{DMO}$, were simultaneously determined in normoglycemic $(n=5)$ and hyperglycemic $(n=6)$ rats with focal cerebral ischemia.

The middle cerebral artery and ipsilateral common carotid artery were occluded by microaneurysm clips in SpragueDawley rats under Halothane anesthesia. Hyperglycemic rats received a $50 \%$ glucose intravenous (IV) infusion. $\left[{ }^{14} \mathrm{C}\right] \mathrm{DMO}$ and $\left[{ }^{3} \mathrm{H}\right]$ nimodipine were infused IV. All studies were terminated by decapitation after 30 minutes of ischemia. The brains were sectioned and autoradiographs of $\left[{ }^{14} \mathrm{C}\right] \mathrm{DMO}$ were obtained using SB5 (Kodak) film. DMO then sublimated from the tissue sections which were then apposed to Hyperfilm (Amersham) to obtain $\left[{ }^{3} \mathrm{H}\right]$ nimodipine autoradiographs.

We observed increased nimodipine binding $(\mathrm{p}<0.01)$ to tissue with $\mathrm{pH}<6.5$. For $\mathrm{pH}<6.6$ our preliminary data shows cortical nimodipine binding to be higher in normoglycemic rats.

In ischemic tissue, regional tissue acidosis correlates with increased nimodipine binding. This is consistent with the hypothesis that one mechanism of acidosis induced injury is through modulation of calcium channel activation. Our data also suggests that increased nimodipine binding is higher in normoglycemic rats than hyperglycemic rats. The reason for this remains to be determined but anaerobic glycolysis may help maintain membrane polarization during the initial phase of cerebral ischemia.

Supported by: Heart and Stroke Foundation of Canada, the Medical Research Council of Canada and Miles Laboratories, Inc.

\section{9.}

The Protective Effects of Superoxide Dismutase and Catalase Alone or in Combination in Repetitive Cerebral Ischemia

D. TRUElOVE, A. SHUAiB and J. KALRA (Saskatoon, Saskatchewan)

During ischemia, the generation of oxygen free radicals (OFRs) may increase neuronal damage. Due to the prolonged reperfusion between insults, OFRs may be partiuclarly harmful under such conditions. In this study we evaluated the protective effects of superoxide dismutase (SOD) and catalase alone or in combination in a repetitive ischemia model in gerbils. Three episodes of ischemia of two minutes each with one hour of reperfusion between insults were used and damage was assessed 7 days after the insult. Superoxide dismutase and catalase were 
infused continuously for 7 days from the onset of ischemia via osmotic pumps. A 0 - 4 point scoring system was used to assess neuronal damage in the cortex, striatum, hippocampus, thalamus, medial geniculate nucleus (MGN) and substantia nigra reticulata $(\mathrm{SNr})$. Superoxide alone showed significant protection in the striatum $(p<0.05)$, thalamus $(p<0.05)$ and the medial geniculate nucleus $(p<0.01)$. Catalase showed significant protection in the striatum $(\mathrm{p}<0.01)$, hippocampus (CAl and CA4) $(p<0.05)$, MGN $(p<0.05), S N r(p<0.05)$ and the thalamus ( $p$ $<0.05$ ). Combination therapy significantly enhanced protection in the striatum ( $p<0.001)$, cortex $(\mathrm{p}<0.05)$, hippocampus (CA1) $(p<0.01)$, thalamus $(p<0.01)$ and the MGN $(p<0.01)$. The use of OFR scavengers is helpful in repeated brief ischemic insults. Catalase alone, or in combination with SOD, offers better protection than SOD therapy alone.

\section{0.}

\section{Hyperthermia Increases Neuronal Damage During Simulated Ischemia in Cell Culture}

\section{R. ISHAQZAY, A. SHUAIB and E. SOCHOCKA (Saskatoon, Saskatchewan)}

There is evidence that during ischemia hypothermia protects neurons both in animals and in cell culture. An increase in brain temperature during ischemia may increase neuronal damage. Fever may frequently occur in patients with acute stroke and therefore the effects of hyperthermia on ischemia needs further study. This study was undertaken to look at the effects of hyperthermia $\left(39^{\circ} \mathrm{C}\right)$, normothermia $\left(37^{\circ} \mathrm{C}\right)$ and hypothermia $\left(32^{\circ} \mathrm{C}\right)$ in a cell culture model of simulated ischemia in cortical astrocytes. Each group consisted of six separate sister cultures. Simulated ischemia was produced by removal of atmospheric oxygen, glucose and glutamine from the medium for the duration of the insult. Neuronal damage was assessed with LDH (units per gram protein) release in the medium. There was significant increase in LDH release in cultures exposed to simulated ischemia under hyperthermic conditions. LDH release was 212 ( \pm 11 , S.D.) in hyperthermic cultures, $135( \pm 3)$ in normothermic cultures and $15( \pm 2)$ in hypothermic cultures. Control cultures not exposed to simulated ischemia showed LDH release of $10( \pm 2)$. Cell culture studies can be used to study the mechanisms for the more severe damage in hyperthermic conditions.

\section{1.}

GABA Agonist "Muscimol" is Neuroprotective in Repetitive Transient Forebrain Ischemia in Gerbils

\section{R. MAZAGRI, S. IJAZ and A. SHUAIB (Saskatoon,} Saskatchewan)

The damaging effects from transient forebrain ischemia may be a result of excessive excitability or loss of inhibitory influ- ences. In the brain, GABA acts as the major inhibitory neurotransmitter and its loss may be an important factor leading to delayed neuronal damage in the substantia nigra reticulata (SNr). In this study, we looked at the protective effects of Muscimol, a GABA A agonist in a gerbil model of repetitive forebrain ischemia. For cerebral ischemia, we used three episodes of two minutes with a reperfusion period of one hour between the insults. Histological evaluations were done seven days after the insult using silver degeneration staining. Muscimol was infused into the third ventricle continuously for seven days beginning just prior to the insult. There were a total of 20 animals, 12 treated with Muscimol and the other 8 serving as controls.

At 7 days, there was significant protection in the cortex $(p=$ $0.007)$, hippocampus [CA1 $(p=0.01)$, CA4 $(p=0.015)$ ], substantia nigra reticulata $(p=0.007)$, striatum $(p=0.049)$ and thalamus $(p=0.012)$. All statistical comparisons were done using nonparametric tests (Mann-Whitney U test). Our study shows that potentiation of inhibitory mechanisms may be important mechanisms of neuronal protection from the effects of repetitive ischemia and the effects are not limited to the SNr. Further studies are needed to better understand their mechanism of action.

82.

\section{U78517F Attenuates Neuronal Damage in Gerbils With Repeated Brief Ischemic Insults}

D. TRUELOVE, A. SHUAIB, J. KALRA and S. RICHARDSON (Saskatoon, Saskatchewan)

Repeated ischemic insults at one hour intervals result in more severe neuronal damage than a single similar duration insult. The mechanism for the more severe damage with repetitive ischemia is not fully understood. We postulated that the prolonged reperfusion periods between the relatively short ischemic insults may result in a pronounced generation of oxygen free radicals (OFRs). In this study, we used a potent OFR scavenger, $\mathrm{U} 78517 \mathrm{~F}$, during repeated ischemia to test if it would reduce the severity of neuronal damage.

Three episodes (two minutes each) of bilateral carotid occlusion were used at one hour interals to produce repetitive ischemia. Two doses of U78517F were given three times per animal one-half hour prior to each occlusion. Neuronal damage was assessed 7 days later in several brain regions with the silver staining technique. The Mann-Whitney U test was used for statistical comparisons.

Compared to controls, there was little protection with a dose of $3 \mathrm{mg} / \mathrm{kg}$. There was significant protection with a dose of 10 $\mathrm{mg} / \mathrm{kg}$ in the hippocampus (CA4) [p $<0.05]$, striatum [p $<$ $0.01]$, thalamus $[p<0.01]$, medial geniculate nucleus $[p<0.01]$ and the substantia nigra reticulata $[\mathrm{p}<0.05]$.

The significant protection with $U 78517 \mathrm{~F}$ with repeated ischemia suggests that OFRs may play a major role in neuronal damage in repeated cerebral ischemia. 
83.

Neocortical Infarction After Temporary Focal Ischemia is Reduced by Moderate Post-Ischemic Hypothermia

\section{Z.G. HUANG, D. XUE and A.M. BUCHAN (Ottawa, Ontario)}

Hypothermia has a dramatic protective effect when instituted intra-ischemically in neocortical ischemia. This study was conducted to test the hypothesis that prolonged post-ischemic hypothermia can benefit cortical infarction in a transient focal neocortical ischemia model. Eighteen male SHR rats underwent 2 hours transient right middle cerebral artery occlusion and permanent right carotid artery occlusion. Upon reperfusion (RP), rats were either maintained normothermically, or were subjected to 6 hours of whole-body mild hypothermia $\left(32-34^{\circ} \mathrm{C}\right)$ or moderate hypothermia $\left(28-30^{\circ} \mathrm{C}\right)$. After 46 hours RP, rats were sacrificed and fixed for frozen sectioning. The volume of cortical infarction was measured and expressed as $\mathrm{mm}^{3}$. Oneway ANOVA was used.

\begin{tabular}{lc}
\hline Groups $(\mathbf{n})$ & Cortical Infarction $\left(\mathbf{m m}^{3}\right)($ mean $\pm \mathbf{S D})$ \\
\hline \hline Normothermia $(6)$ & $175.4 \pm 22.7$ \\
Mild hypothermia (6) & $149.4 \pm 38.7$ \\
Moderate hypothermia (6) & $121.8 \pm 56.5^{*}$ \\
\hline
\end{tabular}

$* \mathrm{p}<0.05$

Post-ischemic but prolonged moderate hypothermia reduces infarct size.

84.

\section{A Novel Strategy for the Treatment of Cerebral Ischemia by Intracellular Calcium Chelators}

M. TYMIANSKI, M.C. WALLACE, I. SPIGELMAN, M. UNO, P.L. CARLEN, M.P. CHARLTON and C.H. TATOR (Toronto, Ontario; Los Angeles, U.S.A.; Tokushima, Japan)

Ischemic neuronal death is associated with an excessive rise in intracellular calcium $\left(\left[\mathrm{Ca}^{2+}\right]_{i}\right)$. We asked whether exogenously applied cell membrane-permeant $\mathrm{Ca}^{2+}$ buffers (MPCB's) could protect neurons against ischemic damage by preventing $\left[\mathrm{Ca}^{2+}{ }_{i}\right.$ increases. In cultured spinal neurons, the MPCB BAPTA-AM lessened $\left[\mathrm{Ca}^{2+}\right]_{i}$ increases and neuronal death by over $75 \%(\mathrm{p}<$ 0.00001 ) following challenges with the neurotoxin glutamate. When applied intravenously to rats, a fluorescent BAPTA-AM derivative reached neurons in cerebral cortex and hippocampus. BAPTA-AM, applied in the same manner, reduced $\mathrm{Ca}^{2+}$-dependent membrane-excitability in hippocampal CAI neurons during intracellular recordings in brain slices taken from treated animals. These effects mimicked those of BAPTA salts delivered directly into neurons through patch pipettes. When adult rats $(230-260 \mathrm{~g})$ were pretreated with MPCBs, they became more resistant to cortical ischemia produced by middle cerebral artery occlusion (MCAO). The protective effects of the chelators were demonstrated in two consecutive, double-blinded, randomized controlled trials $(\mathrm{n}=10$ and 38 rats, respectively). BAPTA and two BAPTA derivatives produced up to $66 \%$ reduction in cortical infarction volume. Our results confirm that $\left[\mathrm{Ca}^{2+}\right]_{i}$ increases contribute to ischemic neuronal damage. The strategy of neuroprotection by intracellular $\mathrm{Ca}^{2+}$ chelation is unique, because it prevents $\left[\mathrm{Ca}^{2+}\right]_{i}$ increases irrespective of the $\mathrm{Ca}^{2+}$ source, thereby obviating the need for therapy with multiple drug combinations to block different routes of $\mathrm{Ca}^{2+}$ influx.

\section{5.}

CGS-19755 is Neuroprotective During Repetitive Ischemia: This Effect is Significantly Enhanced when Combined with Hypothermia

\section{A. SCHUAIB, S. IJAZ, R. MAZAGRI and A. SENTHILSEL- VAN (Saskatoon, Saskatchewan)}

In small animals the damaging effects of repetitive ischemia are more severe than a similar duration single insult. The more severe damage may be a result of prolonged release of glutamate. We report the protective effects of CGS-19755 (an NMDA receptor blocker), or CGS-19755 in combination with mild hypothermia, in a gerbil model of repetitive ischemia. We used three minutes of forebrain ischemia and repeated it three times at one hour intervals. Damage scores were assessed 2 days and 7 days after the insult. At 2 days, CGS-19755 treated animals showed significant protection in the cerebral cortex, hippocampus (CA 1 and $\mathrm{CA} 4$ ), striatum and the thalamus. At 7 days, neuronal protection was seen in the cerebral cortex, hippocampus (CA1 and CA3), striatum and medial geniculate nucleus. The protective effects of CGS-19755 were present when the medication was started before or immediately after the first ischemic insult. When CGS-19755 was combined with mild hypothermia the effects of repetitive ischemia were completely abolished in all but one gerbil. Significant protection was seen in the cerebral cortex, hippocampus (CAl and CA4), striatum, medial geniculate nucleus, thalamus and substantia nigra reticulata. The use of NMDA receptor blockers may protect the brain in repetitive ischemia. Combination with hypothermia may further enhance this protection.

\section{6.}

Protective Effects of Hypothermia During Simulated Ischemia is Dependent on the Duration of the Insult in Cell Cultures

R. ISHAQZAY, A. SHUAIB, W. CODE and E. SOCHOCKA (Saskatoon, Saskatchewan; Duncan, British Columbia)

Mild hypothermia results in considerable neuronal protection in transient cerebral ischemia in small animals. This effect has also been documented in cell cultures. This study was undertaken to determine the window of hypothermic protection in neurons and astrocytes from the effects of simulated ischemia in a cell culture model. We used LDH release in the medium as the indicator for neuronal damage. Experiments were done in groups of six cultures at two temperatures $\left(37\right.$ and $\left.32^{\circ} \mathrm{C}\right)$ and at 
varying duration of ischemia. Our study reveals that with one and three hours of ischemia, there is significant protection in cerebellar neuronal cultures. With cortical neurons, protective effects of hypothermia are evident for longer intervals of 9 hours. Astrocytes showed the protective effects of hypothermia for as long as 12 hours. With ischemic insults of longer duration the protective effects of hypothermia disappears. The protective effects of simulated ischemia are time dependent in cell culture. Hypothermia offers a variable window of opportunity for intervention by other pharmacological methods.

\section{7.}

\section{Delayed Post-Ischemic Ampa Receptor Blockade Reduce Hippocampal CA1 Neuronal Injury}

\section{H. LESIUK, K. BARNES and A.M. BUCHAN (Ottawa, Ontario)}

NBQX, a competitive antagonist of the AMPA class of glutamate receptor, was used to examine the effects of post-ischemic blockade of AMPA receptors, in the 2-vessel occlusion (plus hypotension) model of transient global ischemia, which reliably induces hippocampal CAl neuronal injury. Male SpragueDawley rats $(n=39)$ were subjected to 10 minutes of cerebral ischemia (under general anesthesia with mechanical ventilatory support and meticulous control of temperature, arterial blood gas composition and fluid balance) and 7 days of reperfusion. Animals were randomly assigned to treatment with 3 doses of NBQX (30 mg/kg ip) 15 minutes apart, beginning either immediately on reperfusion or after a delay of 8 hours; control animals received equivalent injections of sterile water. After 7 days, animals were sacrificed and histologic sections of hippocampus prepared. CAl neurons of the septal hippocampus were counted and the ratio of damaged to total neurons calculated. The percentage of damaged CA1 neurons in the pooled control treatment group $(n=20)$ was $69.2 \pm 16.5 \%$ (mean $\pm s d)$. In contrast, CA1 damage in animals treated with NBQX immediately on reperfusion $(n=10)$ was reduced to $11.5 \pm 13.1 \%$ and in animals treated only after 8 hours of reperfusion CAl damage was still reduced to $49 \pm 25 \%$. Thus NBQX treatment resulted in dramatic neuronal protection when administered at reperfusion ( $p<0.0001$; ANOVA, Neumann-Keuls intergroup comparison test), and, even when administration was delayed for 8 hours, significant protection was still achieved $(p<0.05)$ in comparison with control treatment.

\section{8.}

Selective Death Can be Prevented 12 Hours After Ischemia by AMPA Blockade

\section{H. LI and A.M. BUCHAN (Ottawa, Ontario)}

Delayed treatment with NBQX, and AMPA glutamate receptor antagonists, was tested in the 4-vessel occlusion model of severe forebrain ischemia which reliably induces hippocampal CA1 necrosis. Adult male Wistar rats $(n=25)$ were subjected to
10 minutes of forebrain ischemia and seven days of reperfusion. NBQX $(30 \mathrm{mg} / \mathrm{kg})$ was injected IP at the time of reperfusion, and following a delay of 6 hours and 24 hours post-ischemia. Damaged CA1 neurons of the dorsal hippocampus in surviving animals $(n=25)$ were counted and the percentage of injured cells calculated.

\begin{tabular}{lc}
\hline Group (n) & \% of CA1 injury (mean \pm SD) \\
\hline \hline Saline (8) & $81 \pm 28$ \\
NBQX & $17 \pm 17^{*}$ \\
$\quad$ immediate (6) & $21 \pm 32^{*}$ \\
$\quad$ delayed 6 hours (5) & $25 \pm 17^{*}$ \\
$\quad$ delayed 12 hours (6) & \\
\hline
\end{tabular}

$* \mathrm{p}<0.001$ Mann-Whitney U test

AMPA receptor blockade delayed by up to 12 hours prevents CA1 injury suggesting a causal relationship between selective injury and $\mathrm{CA}++$ entry through this glutamate regulated but ischemia-modified ionophore.

\section{Poster Presentations}

\section{I - 1. PEDIATRIC NEUROLOGY}

P1.

Newborn Apnea Caused by Neurofibroma at the Carniocervical Junction

D.B. CLARKE, J.-P. FARMER, G.V. WATTERS, G. ROULEAU and J.L. MONTES (Montreal, Quebec)

Newborn apnea occurs for a variety of reasons, including central hypoventilation, a unique case of which is described below.

A term baby, born into a family with a suspected history of neurofibromatosis type I (NF1), was completely apneic at birth. The authors report, for the first time, the finding by magnetic resonance imaging (MRI) of a neurofibroma at the craniocervical junction causing upper cervical cord and lower brainstem compression. The MRI detection and subsequent subtotal resection of the craniocervical junction neurofibroma resulted in the successful extubation of a previously ventilator-dependent patient. Subsequently, the patient developed an upper respiratory tract infection and was reintubated. Resection of the neurofibroma was completed and hydrocephalus was treated with a shunt. The patient, unable to be weaned off of the respirator, was extubated and expired shortly thereafter, at the age of five months. The severity of the clinical presentation was very unusual for NF1 and the family is currently undergoing genetic testing.

The authors suggest that in newborns with unexplained apnea MRI of the craniocervical junction is indicated. Patients with less compromised cervico-medullary function or afflicted by less aggressive forms of NF1 may benefit in a permanent fashion from a surgical decompression. 
P2.

\section{Tuberculosis of the C.N.S. in Children}

\section{N.J. LOWRY and T. TAM (Saskatoon, Saskatchewan)}

We report the clinical presentations, radiological findings and long-term outcome of 11 cases of pediatric C.N.S. T.B. diagnosed and followed over a 10 -year period. They are grouped into 3 clinco-pathological types: 7 meningitis, 2 tuberculomas and 2 cases of myeloaradiculitis. Diagnosis was late in all cases - the mean duration of systemic symptoms, prior to treatment, in the meningitis cases was $4 \mathrm{I}$ days and of C.N.S. symptoms 19 days; 6 of the 7 meningitis cases had abnormal C.T. brain scans; 1 died, 4 have major C.N.S. deficits and 2 are intact. In contrast, both patients with Tuberculomas have made a perfect recovery. The 2 cases of T.B. myeloradiculitis had unusual presentations with "lightening" abdominal pains and acute urinary retention, they both were complicated by the late development of syringomyelia. We propose the early use of steroids to reduce the likelihood of this complication.

\section{P3.}

\section{Ganglioglioma Presenting as a Vascular Lesion in a 10-Year-} Old Male: A Case Report

G.H. BALTUCH, J.-P. FARMER, K. MEAGHER-VILLEMURE, A. O'GORMAN and J.-L. MONTES (Montreal, Quebec)

We present a case of a 10-year-old male who was admitted with a first onset generalized seizure, and a history of headaches. CT scan and Gadolinium enhanced MRI demonstrated a large inhomogenous contrast enhancing mass of the left frontal lobe, with large cystic component. Cerebral angiography revealed the lesion to be highly vascular, and fed entirely by the internal carotid artery system. The patient underwent craniotomy and the lesion was removed in toto. The neuropathology was a ganglioglioma. We have reviewed the literature; gangliogliomas often present in the second and third decade, are known to have cystic components and are contrast enhancing on CT and MRI, but are classically known to be avascular on angiography. We present a previously unreported case of a markedly vascular ganglioglioma; we conclude that these tumours should be included in the differential diagnosis of vascular supratentorial lesions.

\section{P4.}

\section{Acute Confusional Episode Following Cranial-Axis Radiation}

M. HALL, D. KEENE, E. HSU, L. GRIMARD and E. VENTURYA (Ottawa, Ontario)

Long-term sequalae of cranial radiotherapy in the treatment of primary childhood brain tumors include decreased scholastic abilities, dementia, central endocrine dysfunction and cerebral vasculopathies. The purpose of this paper is to draw attention to a specific syndrome of acute intermittent confusional state occurring several years after completion of radiotherapy.

Case L.L. was diagnosed at age 10 years as having an ependymoma of fourth ventricle which was partially resected followed by cranial cervical axis with boost to posterior fassa. At age 13 years he presented with acute onset "dizziness" followed by aggitation, inability to speak but no new lateralized CNS signs. This event spontaneously resolved after several days. MRI scan of the head at that time was reported to show bitemporal parietal ischemia changes.

Case P.V. was diagnosed at age 5 years as having PNET of cerebellar which was partially resected followed by cranial spinal radiotherapy and chemotherapy. At age 8 years he presented with recurrent episodes of aggitation, inability to speak, and alternating hemisphere with spontaneous resolve within a few days.

Case K.H. was diagnosed at age 16 years as having thoracic spinal cord astrocytoma grade III/IV which was partially resected followed by radiotherapy to cranial axis with overlap of fields in posterior fossa region and chemotherapy. At age 18 years he presented with recurrent dizziness, difficulty speaking, headaches and aggitation. Cerebral angiogram showed narrow cerebral vessels.

All 3 patients received cranial radiotherapy and developed a few years later acute confusional episodes. We postulate that this was due to ischemia resulting from a vasculopathy secondary to radiotherapy.

P5.

Adult Behaviour of Ependymoma Presenting as a Brainstem Tumor

D. KEENE, E. HSU, E. VENTURYA, E. NIZALIK, C. JIMENZ and L. GRIMARD (Ottawa, Ontario)

Brain stem tumors comprise up to 10 to $15 \%$ of all CNS tumors in childhood. The usual histology is an astrocytoma or glioblastoma. As biopsy of pontine tumors is no longer encouraged diagnosis is only made upon autopsy. We wish to report one patient in which ependymoma presented as a very aggressive brainstem tumor.

H.V., a 3-year-old female, presented with acute right hemiparesis. CT scan and MRI of the head confirmed the presence of a large intrapontine mass in a normal fourth ventricle. The patient underwent standard radiotherapy followed by Cisplatinum. There was initial improvement of presenting symptomatology. Three months after diagnosis she suddenly developed a flaccid diplegia. CT scan of the head was unchanged. MRI with Gadolinium enhancement of the spinal area did not show any spinal lesions. Within a month she was quadriplegic. At the time there was no evidence of new cranial nerve dysfunction. She died 5 months after the initial diagnosis. Autopsy showed an ependymoma mainly of the brain stem region with minimal fourth ventricle involvement. There was metastatic spread to the frontal and temporal lobes and spinal axis with accentutation in the distal part of the spinal cord leading to vascular compromise. 
This case is unusual in that

1) the occurrence of the ependymoma into the pons rather than the cerebellum.

2) clinical presentation of "transverse myelitis" in a patient with brainstem glioma.

P6.

Primary Hypothyroidism Presenting with Pseudotumor Cerebri and Hyperplasia of the Pituitary

C. ADAMS, H.J. DEAN, S.J. ISRAELS, P. SHUCKETT, A. PATTON and D. FEWER (Philadelphia, U.S.A.; Winnipeg, Manitoba)

A 12 year 9-month-old girl presented with a history of polyuria polydipsia, headaches, anorexia and vomiting. She had a longstanding history of obesity and was premenarchal. The findings on examination were a diffusely enlarged thyroid, bilateral papilledema and a minimally increased right blind spot. Endocrine investigation demonstrated primary hypothyroidism. Head CT scan showed a hypodense mass in the region of the pituitary with an enhancing rim and suprasellar extension. Lumbar puncture had an opening pressure of $36.5 \mathrm{~cm}$ water and after removal of $7 \mathrm{cc}$ a closing pressure of $22 \mathrm{~cm}$. The diagnosis was primary hypothyroidism, secondary pituitary hyperplasia and co-existant pseudotumor cerebri. Her hypothyroidism was treated with L-thyroxine and the pseudotumor cerebri with dexamethasone. Within 2 weeks her headaches had resolved and her CSF pressure was normal. At 8 weeks she was euthyroid and the hypodense mass was significantly reduced in size. At 20 months head CT scan showed a normal pituitary gland. This demonstrates the association of pseudotumor cerebri with primary hypothyroidism prior to thyroxine treatment. The degree of pituitary hyperplasia, appearing similar to a pituitary tumor, shows the importance of endocrine investigation prior to consideration of pituitary surgery.

\section{P7.}

Unusual Combination of Marfan- and Klippel-Feil Syndrome Complicated by Severe Basilar Invagination

\section{M.I. STEINLIN, S. BLASER and R.H.A. HASLAM (Toronto,} Ontario)

Marfan syndrome (MS) is a connective tissue disorder associated with typical skeletal, cardiac and ophthalmological abnormalities and results from a gene deletion on chromosome 15 . Klippel-Feil syndrome (KFS) is a skeletal abnormality with fusion of cervical vertebral bodies, occasional platybasia and as a severe complication, basilar invagination.

We present a 14-year-old boy with dysmorphic facial features, dislocated lenses and arachnodactyly who experienced progressive ataxia, nystagmus and hearing loss over a 6-month period. Typical skeletal, ophthalmological and cardiac diagnostic criteria for MS were present. Plain films, 3DCT and MRI evaluation demonstrated KFS with fusion of the first and second cervical vertebrae, platybasia and severe brainstem impaction by the $\mathrm{C} 1$ vertebra and odontoid process associated with compression of basilar artery resulting in cerebellar infarction. Marked tonsillar ectopia to the level of $\mathrm{C} 5 / \mathrm{C} 6$ added to the neurological compromise. The patient has subsequently undergone surgical procedure for decompression and stabilization.

The combination of MS and KFS has been described 20 years ago in one previous patient only. We speculate that the association of MS and KFS might be the result of an enlarged mutation at the location of the gene for MS.

P8.

\section{The Home Video Camera as a Diagnostic Tool in Child Neurology}

\section{R.I. MUNN (Toronto, Ontario)}

The diagnosis of both epileptic and non-epileptic paroxysmal disorders is usually based on the parents description of the event. As well, neurologic disorders resulting in developmental regression are often suspected after reviewing the child's developmental history. Recently, the home video camera has become widely available to many famalies. Parents can now make a video-tape of their child showing a paroxysmal event or an earlier stage in the child's development.

I evaluated this tool in the diagnosis of childhood neurological disorders. Between Aug. '92 and Jan.'93 eleven parents were asked to either make a video of their child or provide a video-tape of an earlier developmental stage. Eight parents were able to oblige: paroxysmal kinesigenic dystonia (1), LandauKleffner Syndrome showing normal acquisition of language milestones (2), myoclonicastatic seizures (1), benign neonatal sleep myoclonus (1), paroxysmal nocturnal dystonia (2), early morning myoclonic jerks in Juvenile Myoclonic Epilepsy (1). One parent did not have access to a camera and the remaining 2 parents were unable to capture the event in question on film.

Home video cameras appear to be useful in the diagnosis of childhood neurological disorders. They are a useful teaching tool and parents seem reassured that the physician really understands the event which they are trying to describe.

P9.

\section{Spontaneous Remission of Nocturnal Paroxysmal Dystonia in a Child}

\section{R.I. MUNN (Toronto, Ontario)}

Nocturnal paroxysmal dystonia (Lugaresi 1981) is a syndrome characterized by brief attacks of dystonic posturing with vocalization which occurs in nightly clusters. Recent reports suggest that this is an epileptic phenomena sometimes of frontal lobe origin. We describe a child with Nocturnal Paroxysmal Dystonia in whom spontaneous remission appears to have occurred.

A 9-year-old boy was well until the summer of 1992 when he developed nocturnal episodes characterized by eye opening, dystonic posturing of the limbs and appearing frightened. The 
episodes lasted several seconds during which time he was unresponsive. He then fell back to sleep. The spells occurred at a frequency of $8-10$ per night and never occurred during the day.

Past medical health and developmental milestones were normal. There was no family history of epilepsy and neurological examination was normal. Interictal EEG showed left temporal spikes. Ambulatory ictal EEG showed 3 - $4 \mathrm{~Hz}$ bilateral delta activity without focal onset. CT scan was normal.

The child was treated with Clobazam to a maximum dose of $15 \mathrm{mg}$ at bedtime. There was no improvement in the seizures and behavioral problems occurred. The episodes stopped abruptly with phenytoin 4 months after onset and the behavioral problem resolved. After one month of treatment the family discontinued the medication. Follow-up 2 months later shows that the child remains seizure free despite the withdrawal of phenytoin.

\section{P10.}

\section{Increase in Brain (ADP) Found in Virus Mouse Model of Reyes Syndrome}

\section{N. PARAMANANTHAN, J.F. DUNN and G.K. BADDA (Oxford, United Kingdom)}

Intravenous injection of $\mathrm{B} / \mathrm{Lee} / 40$ virus into $\mathrm{Balb}-\mathrm{c}$ mice induced most symptoms of Reyes Syndrome. We used ${ }^{31} \mathrm{P}$ NMR to detect alterations in phosphocreatine (PCr), ATP, inorganic phosphate (Pi), phosphomono- and diesters (PME, PDE) and $\mathrm{pH}$ in vivo, together with metabolite measurements in brain homogenates to study metabolism in the diseased brain.

${ }^{31} \mathrm{P}$ - NMR spectra were obtained from anaesthetized mice between 28 and 32 hours after viral injection. The brains were rapidly frozen for metabolite assays. Results were significant if $\mathrm{p}<0.05$ (t-test).

Creatine and ATP content together with $\mathrm{pH}$ were unchanged. A statistically significant increase existed in the PME/PDE ratio while PME/ATP was unchanged, inferring (PDE) had decreased. The Pi/ATP and PCr/ATP ratios had significantly decreased. Using the creatine kinase equilibrium, free (ADP) was found to be significantly increased.

These results are consistent with a loss of $\mathrm{Pi}$ in the brain resulting in a decline in (Pi). The increased (ADP) could be a strategy to maintain a constant phosphorylation potential.

\section{P11.}

\section{Alexander's Disease: An Unusual Presentation}

\section{C.L. PRIDMORE and J. KOBAYASHI (Toronto, Ontario)}

We describe an infant with histologically proven Alexander's disease (AD), who presented with regression and rapid head enlargement, with radiololgical evidence of aqueductal stenosis. Computed tomographic (CT) brain scans later showed hypodensity of cerebral white matter, mainly in the frontal lobes, and magnetic resonance imaging (MRI) scans demonstrated extensive high signal intensity in the cerebral white matter and brainstem. This combination of clinical and radiological abnormalities strongly suggested AD. Autopsy confirmed the presence of aqueductal stenosis, secondary to the accumulation of large numbers of Rosenthal fibres in the brainstem. Alexander's disease should be considered in the differential diagnosis of aqueductal stenosis in infants, particularly when accompanied by regression or seizures.

P12.

\section{Brain Dysgenesis and Inborn Errors of Metabolism}

\section{B. BEN-ZEEV, D. CHITAYAT, B. ROBINSON and K. SILVER (Toronto, Ontario; Montreal, Quebec)}

The neuropathological effect of inherited metabolic disorders is the result of "intoxication", "energy deficiency" or both. While intoxication does not usually have an intrauterine effect due to the ability of maternal metabolism to clear the toxic compounds, an abnormality in either energy production or utilization may affect embryogensis and results in congenital abnormalities. The organ most sensitive to energy deficiency is the brain, resulting in brain dysgenesis.

We present a newborn female with microcephaly, dysmorphic facial features and brain dysgenesis who developed infantile spasms and severe psychomotor delay. Investigations revealed lactic acidosis due to partial PDH deficiency with an abnormality in the El complex.

Review of the literature revealed 17 inherited metabolic disorders associated with brain dysgenesis. We discuss the pathological findings and the importance of the affected pathways in normal brain development. The detection of brain dysgenesis on pre- or post-natal neuro-radiological investigation should prompt a metabolic workup on amniotic fluid, fibroblasts, and body fluids, allowing accurate recurrence risks to be defined.

P13.

\section{Spinal Dysraphism State: A Progressive Disorder}

\section{F.B. MAROUN, J.C. JACOB and W.D. HENEGHAN (St.} John's, Newfoundland)

In the careful follow-up of children treated for spinal dysraphism at birth a new entity has emerged and that is progression of symptoms after the initial lesion has been diagnosed and treated at birth.

Twelve such patients were identified with progressive clinical symptomatology increasing between 3 years and 14 years of age. Eleven of those patients were operated for myelomeningocele and 1 patient with neurodermosinus. Their symptoms included progressive spinal deformity, increased bladder and bowel dysfunction, increased leg weakness and pain with progressive foot deformity and spasticity.

Surgical treatment consisted of an array of multiple surgical procedures. The recognition of the continuous changes in the dysraphic state is extremely important since this group is one of the few congenital CNS dysfunctions that are surgically treated. 
P14.

\section{Giant Pigmented Nevi in Children}

\section{KEENE, E. HSU and E. VENTURYA (Ottawa, Ontario)}

CNS tumors in association with some of the neurocutaneous syndrome as well (i.e., neurofibromatosis, Tuberosis sclerosis, Hippel Lander Syndrome). One syndrome often forgotten, but we feel just as important is giant pigmented nevi or bathing trunk nevi syndrome of children.

Over the past 18 years, two children with giant nevus syndrome have been seen by the neuro-oncology service because of primary brain tumors. Both children were pre-adolescent, one with cystic melanotic medulloblastoma of the cerebellum and the second with malignant melanoma in the temporal lobe. Both children died about 3 years from the time of initial neurological symptoms.

S.T., a 9-year-old male presented with increased ICP and a cystic melanotic medulloblastoma was resected from the cerebellum. There were multiple local recurrences in spite of craniospinal radiation, brachytheraphy and chemotherapy. He succumbed to the disease after extensive metasis occurred within the cerebrospinal axis and the skin over incision sites.

J.L. was 9 when she presented with partial complex seizures. CT of the brain showed small calcification of the hippocampal gyrus. She was lost to follow-up. Four years later she returned with headaches. Incomplete resection of a malignant melanoma from the mesial temporal lobe was followed by ICE chemotherapy. There was very rapid proliferation of cells in the CSF and she survived only 3 months after the surgery.

Malignant changes of the skin lesions in giant pigmented nevus occurs in up to $30 \%$ of the patients any time from birth to childhood. Since surgical resection is impossible in many cases, careful follow-up is important to detect onset of malignant melanoma. Rarely, the primary site of involvement may be in the leptomeninges and patients may present with neurological symptoms.

\section{P15.}

\section{Aicardi Syndrome: (AS) Natural History and Predictors of Severity}

D.L. MacGREGOR, A. MENEZES, and J.R. BUNCIC (Toronto, Ontario)

Aicardi Syndrome is defined by a clinical triad of infantile spasms, agenesis of the corpus callosum (partial or complete) and pathognomoic choriorentinal lacunae. Almost all patients are girls (X-linked dominant) with severe cognitive-physical handicaps and epilepsy.

Fourteen patients with AS were reviewed to consider the association by descriptive analysis of 30 features (including ocular findings, seizures, EEG, neuroimaging studies and neurological functioning) at presentation with outcome measures mobility and cognitive scores were plotted. Spearman's rank correlation (Proc CORR) was calculated when the relationship could be approximated by a straight line. Life table estimates were obtained (BMD P1L).
Using mobility as a measure of disease severity, worse prognosis was best correlated to larger lacunar size. No other clinical features were predictive. Estimated survival rate at 6 years was $75 \%$; at 15 years was $40 \%$.

The ocular findings were predictive of severity in AS; a life table could be constructed for girls with this syndrome.

P16.

Werdnig-Hoffman Disease is a Wide-Spread Disease of the Central Nervous System

S.D. LEVIN, S. LUDWIN, H.R. MACKANJEE and D.A. RAMSAY (London, Ontario)

Case One A male 2nd twin was born at 29 weeks gestation with a birth weight of $1335 \mathrm{~g}$. Respiratory distress syndrome was treated with ventilation for 1 month. By 2 months of age he was active and alert but hypotonic. He became progressively more hypotonic and weak although cranial nerve function remained intact. Extensive investigations including EMG revealed no abnormality. He became ventilator dependent. Muscle biopsy showed severe widespread atrophy of muscle fibres.

Case Two A male infant was born by cesarian section at 36 weeks gestation, weighing $2225 \mathrm{~g}$. He was ventilator dependent from birth. Clinical examination showed muscle weakness (including face), hypotonia and areflexia, joint contractures, micrognathia and cleft palate. EKG, CPK, cranial ultrasound were normal. EMG and nerve conduction showed no activity. Muscle biopsy showed evidence of severe denervation.

At autopsy, both patients had severe atrophy of anterior nerve roots with widespread loss of anterior motor neurons from all levels of the spinal cord. Similar changes were seen in cranial nerve nuclei III, IV, V, VI, VII and $X$ as well as the nucleus ambiguus. There was marked loss of neurons of the globus pallidus and thalamus. In case 2 , the putamen, thalamus and dentate nuclei were also involved.

Werdnig-Hoffman disease affects not only the anterior horn cells but all cranial motor nerve nuclei and the deep nuclei of the cerebral hemispheres and cerebellum.

P17.

Rapid Progression in Severe Congenital Muscular Dystrophy (Non-Fukuyama and Non-Cerebro-Ocular Dysplasia Type) with Abnormal Central Myelination and Developmental Delay

A.E. SLOANE, I. TEIN, V. JAY, D. ARMSTRONG and E.G. MURPHY (Toronto, Ontario)

Cerebral dysgenesis associated with congenital muscular dystrophy (CMD) occurs in the Fukuyama (F-CMD) and cerebro-ocular dysplasia (CO-CMD) types. We describe a distinct, rapidly-progressive CMD in a 7-month-old Caucasian girl with developmental delay and abnormal central myelination on neuroimaging. 
Distal joint contractures, hypotonia, and weakness were evident at birth. Apgar scores were low. At 3 weeks, creatine kinase was $2700 \mathrm{U} / \mathrm{l}$. Electromyography demonstrated myopathic features. Muscle biopsy (at 4 weeks) revealed scattered degenerating and regenerating fibres without other changes of muscular dystrophy. Head CT scan revealed mild attenuation of white matter in the left occipital horn.

At 7 months there was severe muscle wasting, hypotonia, marked weakness and areflexia. Head circumference had dropped from the 50th- to 25 th-centiles. Ophthalmological examination was normal. Creatine kinase was 543 U/l. Repeat muscle biopsy revealed a severe muscular dystrophy with extensive necrosis and regeneration. Immunohistochemical staining for dystrophin was normal. Head CT showed severe diffuse white matter attenuation. MRI confirmed abnormal central myelination and absence of cerebral dysgenesis.

In conclusion, this represents a case of CMD (distinct from F-CMD and CO-CMD), with rapid progression of muscle pathology, abnormal central myelination, and absence of cerebral dysgenesis, and expands the clinical spectrum ov CMD.

P18.

\section{Inter-Observer Agreement in the Diagnosis of Childhood Headache}

J. WOLSTEIN, S.S. SESHIA, P. HAESE, C. ADAMS, F.A. BOOTH and J.D. REGGIN (Winnipeg, Manitoba)

We prospectively assessed inter-observer agreement in the diagnosis of recurrent headache histories of 40 children. Randomly selected headache histories of 40 children (age 4.3 to 17.8 years, median 10.4 years) were given to four pediatric neurologists. One or more headache types could be checked off on a data sheet listing the main types recognized by the International Headache Society (IHS) and an additional one: "combined migraine and tension-type headache".

Percentage agreement and kappa statistics were used to measure agreement between six combinational pairs of neurologists yielding 240 pairs of diagnoses. Criteria for agreement were met if both neurologists in a pair diagnosed identical headache types in the same patient. Agreement ranged from $45 \%$ to $78 \%$, kappa values from 0.20 to 0.59 for the six pairs. There were $138(75 \%)$ agreements of the 184 diagnostic pairs involving single headache types only; there were two (4\%) agreements of the 56 pairs involving multiple headache types.

The IHS suggested that patients may have multiple types of headache and that all types be classified. We suggest that the option for diagnosing more than one headache type from the same history may reduce inter-observer agreement.
P19.

\section{Brief Solution Focused Therapy (BSFT) for Interactible Migraine in Children}

\author{
D.L. MacGREGOR, H. MAJ and L.M. LACH (Toronto, \\ Ontario)
}

A follow-up survey of 11 children ( 8 males) with intractible headaches (migraine without aura) was done to evaluate the effectiveness of BSFT (Fam Syst Med 1984; 2: 125-136). Initial assessment was done by a paediatric neurologist and referral was made for this therapy following failure of the standard analgesic treatment in all and prophylactic failure in 4 children. Subjects received a maximum of four therapy sessions and completed a survey questionnaire six months later to compare their pre- and post-treatment status.

All participants (and their parents) reported improvement (up to $70 \%$ ) in coping with headaches using a 10 point rating scale with reduction of headache frequency and secondary symptomatology.

The principle of this therapy is based on the theory that children with intractible headaches have developded coping mechanisms which are either unrecognized and/or under utilized. The main elements include (1) gaining an understanding of the child's and family's perception of the origin of the symptoms, (2) identification of strategies currently being utilized to cope with the pain and (3) enhancement and strengthening of those skills to be employed at the onset of headache.

We conclude that BSFT provides a useful adjunct to currently employed methods for the treatment of intractible migraine in children.

\section{P20.}

\section{Familial Congenital Corneal Anaesthesia}

\section{J.S. KOBAYASHI, M. CLARKE and T. SULLIVAN (Toronto, Ontario)}

Congenital corneal anaesthesia is a cause of severe corneal ulceration and scarring in childhood. Although uncommon, it may be underdiagnosed when present as an isolated entity. Measures such as the use of elbow splints and tarsorrhaphy may be necessary to prevent visual loss. In rare instances, the condition may be inherited. A family is presented with autosominal dominant isolated congenital corneal anaesthesia. The mother was noted to have corneal scarring at 6 years of age. She was found to have bilateral dense corneal anaesthesia with normal tear production and normal cutaneous sensation. Two daughters, ages 4 and 2, had bilateral punctate corneal epithelial erosions. Corneal sensation was absent in both eyes but normal sensation elsewhere in distribution of division one of the trigeminal nerve. Systemic associations and treatment of the condition will be outlined. 
P21.

\section{Seizure Pattern and Electroencephalographic Changes in Patients with Lissencephaly}

\author{
B. KOO, J. KOBAYASHI, S. BLASER and P.A. HWANG \\ (Toronto, Ontario)
}

We reviewed the seizure pattern and electro-encephalographic (EEG) changes in 15 patients with lissencephaly. All patients are severely mentally delayed and hypotonic. Chromosomal studies are normal in all classes. Seven have microcephaly and five have associated brain malformations ( 2 absent corpus callosum, 1 parieto-occipital encephalocele, 2 pachygyria).

All patients have one or more forms of seizure with age of seizure onset ranging from neonatal period to 12 months. Seizures include neonatal (4 cases), generalized tonic-clonic ( 8 cases), partial seizures ( 5 cases), myoclonic seizures ( 4 cases) and infantile spasms ( 3 cases).

In addition to diffusely abnormal and disorganized background characteristic EEG changes include high amplitude slow spike and waves, generalized high amplitude fast beta or alpha activity, paroxysmal runs of high amplitude slow $2-4 \mathrm{~Hz}$ activity and poorly defined sleep changes. Multifocal spikes are frequently present with maximal distribution over posterior temporo-parieto-occipital regions. Hypsarrhythmia is observed in the three patients with infantile spasms.

\section{P22.}

\section{Abnormal SEPs in Children Undergoing Rhizotomy Procedure}

\section{L.J. MacMILLAN, G. MURPHY, J. VAJSAR and M. TAYLOR (Toronto, Ontario)}

Selective dorsal rhizotomy is performed to reduce the spasticity suffered by some children with cerebral palsy. It can improve gait function in those with diplegia and in cases of quadraplegia, can ease daily care by improving posture and positioning. Somatosensory evoked potentials (SEPs) were recorded in 29 patients (19 boys, 10 girls) pre-operatively, from posterior tibial nerve in all 29 and median nerve in 23 . The mean age was 5.25 years.

Median nerve SEPs were normal in the 23 cases studied. Posterioir tibial SEPs were normal in $18(62 \%)$, abnormal in 6 (20\%) and absent in $4(13 \%)$. In some cases the posterior tibial nerve SEPs showed asymmetries of latency and/or amplitudes, as well as unusual morphologies. It is not clear if these findings were due to underlying pathology, or to the difficulty in testing these young disabled children. Six patients with normal SEPs pre-op were retested post-operatively with posterior tibial nerve SEPs (mean $4.5 \mathrm{wks}$ ). The cortical SEPs were absent in $5 / 6$ patients. About $50 \%$ of afferent fibres and cut during the rhizotomy (S2-L4); perhaps the fibres left are not sufficient to propogate the stimulus to the cortex. Long-term studies are needed in these children to determine if these findings are transient.
P23.

Monitoring of Cerebral Oxygenation During Cardiac
Bypass Sugery by Near Infrared Spectroscopy

M. MOODLEY, K.E. POHL, E.H. ROLAND, A. MacNAB and A. HILL (Vancouver, British Columbia)

Neurological dysfunction is a common complication of cardiac bypass surgery in childhood. The new, noninvasive technique of near infrared spectroscopy (NIRS) permits direct, continuous intraoperative monitoring of cerebral oxygenation of cerebral blood flow and volume. The purpose of the study was to monitor cerebral oxygenation and hemodynamics (by measurement of oxyhemoglobin, deoxyhemoglobin and cytochrome oxidase) during cardiac bypass surgery in children using NIRS.

The study population comprised 14 children (mean age 3.2 years) who underwent hypothermic cardiopulmonary bypass and circulatory arrest. In 10 children, during hypothermia and bypass, there was gradual increase in cytochrome oxidase levels which decreased rapidly to baseline levels during circulatory arrest. At times of surgical complications, e.g., massive blood loss, NIRS demonstrated abrupt decrease in cerebral oxyhemoglobin level, prior to the recognition of distributed hemodynamics by conventional monitoring techniques.

These data suggest that NIRS is of value for intraoperative monitoring of cerebral hemodynamics of children during cardiac bypass surgery who are at risk for hypoxic-ischemic insult.

P24.

\section{Suicide and Pediatric Epilepsy}

M.L. SMITH, D. MINDEN, C. STEVERANGO, D. SMITH and M. WASDELL (Toronto, Ontario; Halifax, Nova Scotia)

One of the most serious consequences of the psychosocial problems associated with epilepsy is an increased incidence of suicide. The suicide rate among epileptic adults has been reported to be 5 times higher than in the general population (Barraclough, 1981). Suicide in epileptic children and adolescents has been examined in only one previous study found a 15 fold increase in suicide risk (Brent, 1986).

The purpose of the present study was to replicate the finding of increased risk of suicide in pediatric epilepsy, by conducting a retrospective chart review of 139 consecutive suicide attempters over a 5-year period who were taken to a pediatric hospital for evaluation and/or treatment. Of these, 5 had a history of seizure disorder, an incidence which represents an 8-fold increase in risk of suicide. Although this figure is not as high as that reported by Brent (1986), differences in seizure types in the two samples may explain some of the discrepancy. Medical and psychosocial variables, including psychiatric disorder, medication, and family dysfunction, which may place the epileptic child at particular risk for suicide, are discussed. 
P25.

\section{Alfentanil Can Induce Epileptic Seizures}

\section{KEENE, D. ROBERTS and F. VENTURYA (Ottawa, Ontario)}

Unlike the adult, the child undergoing surgery for the removal of epileptic focus often need to be done under general anaesthesia. The aneasthetic agent used must be quick acting; of short duration; and not interfere with ECOG recording. For these reasons narcotic relaxation techniques have been used.

Afentanil is a new potent short acting narcotic agent that can be used as an anaesthetic adjunct. It has not been reported to be associated with activation of seizures. For these reasons, it has been tried at our institution.

Nine consecutive patients ( 4 females, 5 males) undergoing surgical resection of epileptic focus have received this drug. All but 1 patient had temporal lobe epileptic focus (the other had parietal temporal focus) standard anaesthetic protocol was followed in all cases.

ECOG was recorded for 30 minutes prior to and after receiving Alfentanil $10 \mathrm{mg} / \mathrm{kg} \mathrm{IV.} \mathrm{3/9} \mathrm{had} \mathrm{clinical} \mathrm{and} \mathrm{electrographic}$ seizures after administration. 7/9 had significant activation of epileptiform activity on ECOG. 2/9 had no change.

Alfentanil may prove helpful in enhancement of intraoperative ECOG recordings of epileptiform abnormalities. Its use in patients with clinical seizures must be with great caution.

P26.

Minor Anomalies in Offspring of Epileptic Women: A Controlled Blind Study

K. SILVER, I. LOPES-CENDES, L. SIAVALAS and E. ANDERMANN (Montreal, Quebec)

An increased number of minor anomalies (MA) in offspring of epileptic women has been reported regardless anticonvulsant (AC) exposure in utero, and a variety of etiologic factors have been implicated including: genetically transmitted features related to the epilepsy and teratogeneity of the ACs. We have assessed 96 offspring of epileptic women: 68 on monotherapy $(\mathrm{CBZ}=39, \mathrm{PHE}=16, \mathrm{VAP}=8$ and $\mathrm{PB}=5), 20$ on polytherapy, 8 on no medication during pregnancy; and 7 controls. MA were recorded by one of us (KS) who was unaware to which group the child belonged. A score was attributed to each child according to the number of MA found. The statistical analyses were performed using ANOVA with correction for multiple comparisons.

Children of epileptic women had an increased number of MA as compared to controls ( 2.15 and 1.6 respectively) regardless the $\mathrm{AC}$ regimen. However, this did not reach statistical significance $(p=0.27)$, probably due to sample size. In addition there was no significant difference in the number of MA associated with different $\mathrm{AC}$ regimens $(\mathrm{p}=0.85)$, indicating that genetic factors may be more important in the cause of MA than the drug exposure.
P27.

\section{Scalp EEG on Three-Dimensional CT Imaging}

H. OTSUBO, D. ARMSTRONG, S. HOLOWKA, P.A. HWANG, A. HUNJAN and H.J. HOFFMAN (Toronto, Ontario)

To identify epileptiform activity corresponding to structural lesions, we created three-dimensional (3-D) imaging of computerized tomography (CT), with concurrent scalp EEG electrodes. In children scalp EEG electrodes were placed according to the International $10-20$ system and used to record an electroencephalogram to detect interictal and ictal epileptogenic foci. Therefore the electrodes where maximum and moderate epileptiform activity were detected interictally or ictally were identified and replaced with physical markers which are detectable on CT. Computer analysis of 3-D imaging with these markers on the skin was mapped by the 3-D imaging system (ISG), and correlated with underlying cerebral structures.

These views are used to make a preoperative assessment of the relationship of intracranial lesions to brain surface structures associated with epileptogenic foci which are demonstrated on the scalp EEG.

An intraoperative 3-D stereotaxic pointing device which accurately locates lesions stored in the 3-D CT imaging system, helps to detect the edge of mass lesions as well as the epileptogenic zone in epilepsy surgical procedures.

P28.

\section{Head Circumference in Offspring of Epileptic Women}

I. LOPES-CENDES, K. SILVER, L. SIAVALAS and E. ANDERMANN (Montreal, Quebec)

We assessed 87 offspring of epileptic women: 66 on monotherapy $(\mathrm{CBZ}=39, \mathrm{PHE}=16, \mathrm{VAP}=7$ and $\mathrm{PB}=4), 10$ on polytherapy, 11 on no medication during pregnancy; and 7 controls. Mean age of examination was 4 years (10 days to 11 years). Head circumference (HC) measurements were performed by one of us (KS) using a flexible steel tape applied above the supraorbital ridges anteriorly and on the occipital prominence posteriorly. In addition, we obtained $\mathrm{HC}$ from birth records of 55 of these children. We compare all measurements with standard values according to age and sex. Microcephaly was diagnosed if $\mathrm{HC}$ was below $2 \mathrm{SD}$. Analysis of variance and a non-parametric test were used to compare all 7 groups; a correction for multiple comparisons was performed in order to analyze specific groups.

There was no difference in the frequency of children with microcephaly among all groups either at birth $(p=0.87)$ of later in life $(p=0.75)$. Comparisons among specific groups also failed to demonstrate any differences. Furthermore the mean $\mathrm{HC}$ at birth was not significantly different for any of the 7 groups $(p$ $=0.90$ ). Although the sample size is not sufficient for an accurate comparison among drug exposure groups, the risk of microcephaly does not appear to be elevated among the offspring of epileptic women. 
P29.

\section{Open-Label Study of Lamotrigine in Children with Intractable Generalized Epilepsy}

\section{CONNOLLY, R. MUNN, M. BERG and K. FARRELL} (Vancouver, British Columbia)

Lamotrigine is a phenyltriazine, which acts presynaptically at voltage-sensitive sodium channels to inhibit the release of glutamate. We describe the efficacy and tolerability of lamotrigine in an open-label, add-on study in 37 children with intractable generalized epilepsy. The epilepsy types included absence epilepsy (3), Lennox-Gastaut syndrome (8), infantile spasms (5), severe myoclonic epilepsy of infancy (2), Baltic myoclonus (1), E.S.E.S. (1), other secondary generalized epilepsies (17). The mean age was 9 years and most children had daily seizures. The seizures had been unresponsive to 2 - 16 antiepileptic drugs.

The daily dose of lamotrigine ranged from $1-8 \mathrm{mg} / \mathrm{kg}$ (mean $3.75 \mathrm{mg} / \mathrm{kg}$ ). The mean follow-up was 4.5 months. The efficacy could not be assessed in 6 children in whom the medication was discontinued due to adverse effects; rash (3), headache (2), sedation (1).

The reduction in seizure frequency was: $>90 \%-4$ patients; $50-90 \%$ - 5 patients; < 50\% - 18 patients; exacerbation - 4 patients. Adverse effects were reported in 14 children: rash (3), headache (4), change in behavior (4), sedation (1), drooling (1), tremor (1). There was no significant alteration in the blood levels of other drugs.

Lamotrigine resulted in a $>50 \%$ reduction in seizures in $24 \%$ of children with intractable epilepsy in this study. Children with Lennox-Gastaut syndrome appeared to be helped particularly.

P30.

\section{In Vivo Magnetic Resonance Spectroscopic Imaging in Rasmussen's Encephalitis}

\section{F. CENDES, D.L. ARNOLD, Y.M. HART and F. ANDER- MANN (Montreal, Quebec)}

Magnetic resonance spectroscopic imaging (MRSI) is capable of determining the spatial distribution in vivo of cerebral metabolites, including $\mathrm{N}$-acetylaspartate (NAA), a compound found only in neurons.

We performed MRSI in a 13-year-old boy with right hemiparesis and epilepsia partialis continua involving the right side of the face secondary to Rasmussen's encephalitis of 7 years duration. MRSI showed a decrease in the relative resonance intensity of NAA over the entire left hemisphere, more accentuated in the periventricular region $(61 \%$ reduction over periventricular region, $49 \%$ reduction over parieto-occipital region and $30 \%$ over fronto-central areas). The NAA reduction was not restricted to the cortex, but was present to an even greater extent in the white matter.

Previous investigators have shown that the in vitro NAA concentration in neocortical tissue from patients with Rasmussen's encephalitis, undergoing surgery, is reduced in proportion to the severity and extent of the histopathological abnormalities (Sutherland and Peeling, Proc Soc Magn Reson Med 1992; 11: 1914). Our findings indicate that MRSI can be used to quantify neuronal and axonal damage in vivo related to the encephalitis, and may be of use in monitoring progression and treatment of the disease.

P31.

Spina Bifida and Stroke - Two Cases

M. VASSILYADI, J.L. MONTES and J.P. FARMER (Montreal, Quebec)

Cerebrovascular accidents (CVA) in two children with repaired myelomeningoceles, decompressed Amold Chiari II malformations, and properly functioning ventriculoperitoneal shunts are presented with review of the literature. The first, a 4year-old boy, became febrile and hemiplegic after status epilepticus. He was found to have sustained an infarct in the right anterior and middle cerebral artery territories. The second, a 15month-old girl, presented with a decreased level of consciousness, acute stridor, and quadriparesis. She was found to have suffered a brain-stem infarct, worse on the right side. In both cases, the CVA occurred acutely with no previous ischemic episodes. Cardiology and Hematology investigations were normal.

The incidence of cerebrovascular accidents in our spina bifida population appears to be significantly higher than that of the general pediatric population. Potential contributing factors to this increased incidence are discussed.

P32.

\section{Cerebral Venous Thrombosis in Childhood}

\section{E. SELLERS, D. KEENE, S. GRAHOVAC and M. POTHOS (Ottawa, Ontario)}

Cerebral venosis thrombosis is an uncommon entity in children, easily recognizable by Magnetic Resonance Imaging (MRI) and can have a benign outcome.

Cerebral venous thrombosis reported in three children. The first case, age 5.5 months, presented with a two-week history of low grade fever, irritability and bulging fontanelle. The second, age 14 months, presented with a four-day history suggestive of viral gastritis (vomiting, irritability and progressive lethargy). Following admission acute hemiparesis became evident. The third child, age 7 years, was admitted to hospital following tonic-clonic seizures preceded by a ten-day history of vomiting, diarrhea and abdominal cramping. Though CT scans were performed on all, the diagnosis was confirmed by MRI. Angiograms were not performed. Spontaneous resolution of clinical findings is reported in all three children without the use of anticoagulation. Follow-up MRI demonstrated recannulization of the thrombosed vessels in two of the cases, follow-up is pending on the third. These cases lend support to the growing literature that suggests that anticoagulation therapy is not necessay in cases of cerebral venous thrombosis. It is also suggested 
that MRI represents the most appropriate, non-invasive method of investigation.

\section{P33.}

\section{A Comparison of Computerized Tomography and Ultrasound Scanning in Neonatal Stroke}

\author{
S. PARNES and D. MARTIN (Ottawa, Ontario; Buffalo, \\ U.S.A.)
}

Neonatal stroke is an important cause of neonatal seizures and neurodevelopmental handicap. The diagnosis is suspected on the basis of seizures and is confirmed by brain computerized tomography (CT) scanning. Neither the diagnostic reliability of brain ultrasound (US) nor the prognostic value of either imaging modality with respect to neurodevelopmental outcome has been established. In this series of 26 consecutive patients with neonatal stroke, the diagnostic and prognostic value of CT and US is compared.

Twenty-three patients had both CT and US within one week of the stroke. In 17, the diagnosis of infarction was confirmed by both US and CT. In 7, US missed infarctions present on CT. In 3, US defined infarctions more clearly than CT did.

Follow-up ranged from 8 months to 7 years. CT abnormalities in 18 patients predicted the development of hemiparesis: 10 were hemiparetic. US abnormalities in 15 patients predicted the development of hemiparesis: 10 were hemiparetic. Four patients had seizures: 7 had cognitive delay. These patients were more likely to have had multifocal infarctions. Overall, CT showed multifocal infarctions more frequently than US.

We conclude that CT is superior to US in the acute diagnosis of neonatal stroke, and that neither prognosticated reliably.

P34.

Post-Traumatic Carotid-Cavernous Fistula in a Child: Case Report

A. BILOCQ, J.P. FARMER, J.L. MONTES, A.M. O'GORMAN, J. RAYMOND and D. ROY (Montreal, Quebec)

Post-traumatic carotid-cavernous fistula in the pediatric population is a rare event. We report the case of a 8-year-old boy presenting with a carotid-cavernous fistula five months after sustaining a blunt head injury. The patient was successfully treated by transfemoral balloon obliteration. A review of literature revealed only 4 cases of post-traumatic fistula occurring under the age of 10 years. Possible mechanisms explaining the low incidence of post-traumatic fistula in the pediatric population are reviewed. These mechanisms include the plasticity of surrounding bone at the skull base and the absence of atheromatous degeneration of the carotid artery. Since the creation of the neurosurgical unit at the Montreal Children Hospital in 1961 this is the only case of post-traumatic carotid-cavernous fistula documented. With an average of 300 head trauma admissions yearly, with 20 to 25 major injuries, this would place the incidence of carotid-cavernous fistual at about 1 per 9,000 head injuries in our hospital population.
P35.

Spontaneous Non-Traumatic Anterior Compartment Syndrome With Peroneal Neuropathy and Favourable Outcome

\author{
A.E. SLOANE, J. VAJSAR and E.G. MURPHY (Toronto, \\ Ontario)
}

Non-traumatic anterior compartment syndrome (ACS) occurs when increased tissue pressure in the anterior compartment of the leg compromises local circulation leading to rhabdomyolysis of the extensor muscles of the foot with resulting foot drop. Injury to the deep peroneal nerve (DPN) in this compartment may occur concurrently.

We describe a 3-year-old girl who developed foot drop 12 days after an acute spontaneous ACS. Computerized tomography findings and creatine kinase of 12,769 I.U. provided diagnostic support. Electromyography showed fibrillations and absence of motor unit potentials on volitional contraction. Conduction velocities of the DPN were $44.2 \mathrm{~m} / \mathrm{s}$ (left) and 45.7 $\mathrm{m} / \mathrm{s}$ (right). The distal latency of the compound muscle action potential recorded from the left extensor digitorum brevis was prolonged to $7.8 \mathrm{~m} / \mathrm{s}$, (right, $3.6 \mathrm{~m} / \mathrm{s}$ ); amplitudes were 0.9 and $9.9 \mathrm{mV}$ respectively. Clinical resolution and improvement in electrophysiological and radiological findings occurred with conservative management.

Whereas muscle dysfunction in ACS due to vascular compromise often warrants decompression to prevent neurological sequelae, DPN neuropraxia can be treated conservatively without fasciotomy, with good outcome. Electromyography is recommended in patients with suspected ACS to recognize an otherwise undetected peroneal neuropathy.

\section{I - 2. NEURO-ONCOLOGY}

P36. Myelopathy Following Intrathecal Methotrexate (MYX) and
Cytosine Arabinoside (ARA-C)

D.R. McLEAN, H. CLINK, R. COATES, Z. AL-KAWI, S. BOHLEGA and S. OMER (Riyadh, Saudi Arabia)

Sporadic cases of myelopathy have been reported following intrathecal (IT) MTX and/or ARA-C. The neuropathology has been described. However, there are no magnetic resonance imaging (MRI) descriptions of this condition.

We report 3 patients who developed myelopathy following intrathecal MTX and ARA-C combined with cranial-spinal irradiation. Several cerebrospinal fluid specimens from each patient showed no viral, bacterial or fungal infection by culture or serological testing.

An early MRI showed minimal changes in 2 patients. In the third patient, an MRI done after several weeks showed increased T-2 signal intensity scattered throughout the lateral columns of the cervical spinal cord. 
Two patients treated with oral corticosteroid showed gradual improvement although one has subsequently deteriorated when intrathecal MTX was reinstituted for central nervous system relapse.

Measures to prevent this devastating complication will be discussed.

P37.

\section{Gelatinase A Activity in Human Glial Tumors}

R.F. DEL MAESTRO, P.C. COSTELLO, I.S. VAITHILINGAM and W.G. STETLER-STEVENSON (London, Ontario; Bethesda, U.S.A.)

Background: Tumor angiogenesis requires the degradation of collagen type IV. The questions addressed in this study were (1) Do U25l cells (derived from a human malignant glioma) release collagen type IV activity? (2) Do these cells contain mRNA for gelatinase A ( $72 \mathrm{kDa}$ type IV collagenase)? (3) Do human malignant gliomas contain mRNA for gelatinase A? (4) What is the distribution of gelatinase A in malignant gliomas?

Methods: Collagenase type IV was assayed by measuring ${ }^{3} \mathrm{H}$ collagen IV degradation. Northern hybridization analysis was used to study gelatinase A mRNA content. Histochemical studies were performed on tumor specimens using a specific antibody to human gelatinase $A$.

Results: $U 251$ release collagen type IV activity and contain mRNA for gelatinase $A$. The mRNA levels for gelatinase A were increased in malignant glial tumor and histochemical studies localized this activity to the tumor edge.

Conclusions: U251 cells release collagenase type IV activity and contains mRNA for gelatinase $\mathrm{A}$. The increased gelatinase A activity of human malignant glial tumors is localized to the growing edge of tumors.

\section{P38.}

\section{Leptomeningeal Melanocytosis in Patients with Neurofibromatosis}

J. ALWATBAN, D. TAMPIERI, A. SALAZAR, H. DUONG and D. MELANSON (Montreal, Quebec)

Leptomeningeal melanocytosis in a rare form of benign proliferation of melanocytes in the meninges. This entity should be differentiated from the malignant meningeal melanomatosis. Their mode of clinical presentation may however, be similar.

Meningeal melanocytosis is known to occur in patients with cutaneous and ocular nevi, the association with neurofibromatosis has not been reported.

The authors describe the CT and MRI findings of meningeal melanocytosis in a patient with neurofibromatosis type I, and discuss the value at these modalities for accurate diagnosis and proper biopsy localization.
P39.

High Dose Rate Fractionated Interstitial Brachytherapy for Recurrent Brain Tumors

J.P. BAHARY, J.G. VILLEMURE, G. MOHR, B.G. CLARK, M.D.C. EVANS, S. LEHNERT, C. PLA and L. SOUHAMI (Montreal, Quebec)

In an attempt to improve local control in patients with recurrent high grade glioma we have recently developed a program at McGill University for recurrent CNS tumors. Treatment consisted of high dose-rate Iridium-192 afterloading brachytherapy. Two patients with recurrent glioblastoma multiforme and one with recurrent gliosarcoma were treated with doses of $500 \mathrm{cGy}$ delivered twice a day for 3 days to a total dose of $30 \mathrm{~Gy}$. Two patients were stable for 7 and 8 months post-brachytherapy and another one showed remarkable regression of his tumor 4 weeks after the implantation.

Technique and results will be illustrated.

P40.

The Stability of Blood Count Changes Induced by Craniotomy With and Without PDT

P.J. MULLER and K. ASHKAN (Toronto, Ontario; Cardiff, Wales)

The non-metallo porphyrins have an effect on the immune system and hematopoetic systems. HPD increased murine marrow cellularity and induced splenic hypertrophy. BCNU induced marrow suppression has been shown to be reversed with HPD pre-treatment.

We have retrospectively compared the pre-operative and post-operative hemoglobin, wbc, and Hct in 63 patients with malignant gliomas treated with intraoperative PDT to an equal number of control patients with the same pathology who did not receive PDT. All cases received steroids. The pre-operative blood counts were taken on the day prior to surgery which was the day of porphyrin administration (Photofrin $2 \mathrm{mg} / \mathrm{kg}$, IV). A summary of the blood counts is given below:

\begin{tabular}{lcccc}
\hline \multicolumn{5}{c}{ PDT Treated } \\
Control & Pre-op & Post-op & Pre-op & Post-op \\
\hline \hline WCB & $9.6 \pm 3.5$ & $16.2 \pm 5.2$ & $8.5 \pm 3.1$ & $14.4 \pm 6.5$ \\
RBC & $4.6 \pm 56$ & $4.1 \pm 0.55$ & $4.5 \pm 0.59$ & $4.0 \pm 0.65$ \\
HBG & $142 \pm 13$ & $128 \pm 15$ & $140 \pm 15$ & $122 \pm 15$ \\
LYMPH & $16.4 \pm 8.9$ & $8.8 \pm 4.1$ & $17.8 \pm 7.9$ & $10.0 \pm 5.5$ \\
\hline
\end{tabular}

No differences were identified in the absolute blood count levels between the PDT and control groups nor in the pre/postoperative blood count ratios. We conclude that a single HPD administration at $2 \mathrm{mg} / \mathrm{kg}$ does not alter the effect of craniotomy stress on the basic clinical blood count profile. 
P41.

Photodynamic Therapy of Supratentorial Brain Tumours: Acute Complications

\section{P.J. MULLER and B.C. WILSON (Toronto, Ontario)}

Photodynamic therapy of cancer consists of the administration of a photosensitizer followed by the photoillumination of the neoplastic tissue. We have used PDT 78 times in the treatment of 77 patients with the following pathology: GBM 36, malignant astrocytoma 21 , malignant mixed glioma 4 , ependymoma 3 , metastases 9 , meningioma 3 , and pituitary adenoma 1 . In $2 / 3$ of the patients the tumour was recurrent.

All patients received Photofrin $2 \mathrm{mg} / \mathrm{kg}$ i.v. 12 - 36 hours prior to surgery. All patients with malignant gliomas or metastases had intraoperative cavitary photoillumination.

The complication rate was as follows:

$\begin{array}{lll}\text { post-operative death: } & 3 & (4 \%) \\ \text { post-operative neurological deficit: } & 5(6 \%) \\ \text { suture infection: } & 2(3 \%) \\ \text { bone infection: } & 2(3 \%) \\ \text { deep vein thrombosis: } & 5(6 \%) \\ \text { minor skin toxicity: } & 3(4 \%) \\ \text { major skin toxicity: } & 1(1 \%) \\ \text { systemic reaction to drug: } & 0(0 \%) \\ \text { "excessive" cerebral edema: } & 8(10 \%)\end{array}$

The 4\% (30 day) operative mortality rate is an acceptable rate for these patient categories. The degree of edema was somewhat greater than that of concurrent non-randomized controls.

\section{P42.}

The Relationship Between Pituitary Adenoma Size and Clinical Presentation, Pathological Type and Operative Risk

\section{P.J. MULLER, K. THAPAR and K. KOVACS (Toronto, Ontario)}

We have extended our previous report on pituitary adenomas by adding cases and reviewing the pathology. Tumour size was measured from CT or MRI as the longest lesional diameter. There were 16 patients with microadenomas $(<1 \mathrm{~cm}$ diameter $)$, 48 with macroadenomas $(1-2.5 \mathrm{~cm})$ and 63 with large pituitary adenomas $(>2.5 \mathrm{~cm})$. The male/female ratios for these groups were $0.2,1.5$, and 2.1 ; and, the mean ages were $37 \pm 12,46 \pm$ 14 , and $53 \pm 16$, respectively.

\begin{tabular}{lcc}
\hline Pathological Type & Number & Mean Size (cm) \\
\hline Corticotroph (densely granulated) & 10 & 1.14 \\
GH adenoma (densely granulated) & 6 & 1.08 \\
GH adenoma (sparsely granulated) & 6 & 2.48 \\
Glycoprotein (undifferentiated) & 2 & 1.60 \\
Glycoprotein (gonadotroph) & 7 & 2.62 \\
Glycoprotein (thyrotroph) & 2 & 2.65 \\
Mised acidophil adenoma & 5 & 2.10 \\
Null cell adenoma & 25 & 3.00 \\
Oncocytoma & 26 & 2.99 \\
Prolactinoma (sparsely granulated) & 30 & 2.35 \\
Silent subtypes & 6 & 2.95 \\
Unclassified & 2 & 3.90 \\
\hline
\end{tabular}

There were no deaths among the microadenomas; and one death in the macroadenoma group as the consequence of a pulmonary embolus. Five died in the large tumour group (3 secondary to hemorrhage and 2 with presumed hypothalamic injury). There were a total of 20 non-lethal complications.

P43.

\section{Stereotactic Surgery in the Poterior Fossa: A Review of 11 Cases}

W.P. NG, A. LOZANO and M. BERNSTEIN (Toronto, Ontario)

We reviewed 11 patients who underwent stereotactic biopsy and/or cyst aspiration for lesions in the posterior fossa under local anesthesia. There were 8 males and 3 females, aged 18 74 years. Three patients had more than 1 lesion. Eight presented with brain-stem compression and motor deficits. The size of the lesions was 2 to $4 \mathrm{~cm}$. Five lesions were in the pons, 4 in the cerebellum, 1 in the midbrain, and one in the cerebello-pontine angle subdural space.

A total of 13 stereotactic procedures were performed in the 11 patients. The BRW frame with transfrontal approach was used 6 times. Transcerebellar approach was used 2 times with the CRW frame and 5 times with the Leksell frame. Biopsy showed 1 primary CNS lymphoma, 3 cases of PML, 2 astrocytomas, and one epidermoid. Aspiration was performed for pontine bacterial abscess ( 3 procedures in the same patient), 1 bacterial cerebellar abscess, 1 astrocytoma cyst, and 1 cerebello-pontine angle subdural tuberculous abscess. All 4 patients who underwent therapeutic cyst aspiration improved clinically.

One patient with PML died from end-stage AIDS, for a 30 day mortality of $9 \%$. No other morbidity was incurred by the other 10 patients.

Posterior fossa stereotaxis allowed for safe and effective biopsy and aspiration of deep-seated lesions in patients for whom the neurological and medical risk of a larger procedure under general anesthetic might be prohibitive. 
P44.

Basic Fibroblast Growth Factor in C6 Astrocytoma: Molecular Characterization of an Autocrine Growth Factor

\section{G.J. REDEKOP and C.C.G. NAUS (London, Ontario)}

Despite agressive therapy including surgery, radiation, and chemotherapy the results of treatment for malignant primary brain tumors remain poor. A fundamental understanding of the molecular mechanisms underlying malignant transformation is required for the development of rational therapeutic strategies.

The authors have used the C6 astrocytoma cell line as a tumor model to study the role of basic fibroblast growth factor (bFGF) using in vitro growth analysis, Northern and Western blotting, and immunocytochemistry. $C 6$ cells produce a $6.0 \mathrm{~kb}$ bFGF mRNA and 2 bFGF protein species with molecular weights of 18 and $22.5 \mathrm{kDa}$. In addition, they have high affinity bFGF receptors through which exogenous bFGF acts as a mitogen, confirming its function as an autocrine growth factor. The C6 astrocytoma is a well-characterized and reproducible model in which to study the molecular mechanisms through which bFGF acts to promote cell growth.

P45.

Third Ventricle Choroid Plexus Neoplasms: Case Report and Review of 85 Cases from the Literature

\section{LI and J.-G. VILLEMURE (Montreal, Quebec)}

Third ventricle choroid plexus neoplasms are rare lesions. The purpose of this review is to gain insight into the management of these lesions, especially in regards to the role of radiotherapy given the risks associated with surgery. We report a case successfully biopsied and treated with fractionated stereotactic irradiation, and review 85 cases from the literature. Data on the presentation, investigations, treatment and outcome of third ventricle choroid plexus papillomas are collected. Even in recent years, surgical morbidity $(50 \%)$ and mortality $(6 \%)$ with total excision high. We present an atypical case of choroid plexus papilloma (CPP) which lacked the usual vascular features on radiologic examination. We are the first to use stereotactic irradiation to treat such a lesion so we briefly review the past use of radiotherapy in the treatment of choroid plexus neoplasms. We suggest stereotactic irradiation as a useful treatment and emphasize the importance of keeping choroid plexus papilloma in the differential diagnosis of third ventricle lesions.

P46.

Gliosarcoma With Osteosarcomatous Features: A Case Report

R.K. MOSEWICH, L. ANG, H. REES, C. McDOUGALL and M. KHAN (Saskatoon, Saskatchewan)

Gliosarcoma is a well described tumor characterized by the presence of both glioblastomatous and sarcomatous elements.
The sarcomatous areas are characteristically fibrosarcomatous in nature. We describe a case of gliosarcoma in which the sarcomatous component was an osteosarcoma.

A 79-year-old right handed lady presented with a 6-week history of progressive headache, left hemiparesis and obtundation. A computed tomographic scan revealed an enhancing, densely calcified mass in the right orbito-frontal area with a significant mass effect. The tumor was removed. At surgery, the tumor was felt to be well circumscribed. It was not attached to bone and was only mildly adherent to the meninges.

The tumor specimen consisted of a soft, irregular, granular tissue surrounding a hard calcified core. Microscopically, there were areas of gliomatous tissue with nuclear atypia, mitotic figures, necrosis and endothelial proliferation. This area stained positive for glial fibrillary acidic protein (GFAP) and vimentin. There were other areas of malignant osteoid formation mixed with mesenchymal elements.

Mixed glioblastoma-osteosarcoma has only rarely been described (Sariento et al., 1979). To our knowledge this is the first described case with detailed immunohistochemistry.

\section{P47.}

\section{Influence of Coumadin on C6 Tumor Growth}

\section{R.F. DEL MAESTRO, I.S. VAITHILINGAM, W. McDONALD and E. STROUDE (London, Ontario)}

Background: C6 cells release 2 types of enzymatic activity capable of degrading collagen type IV. A novel large molecular weight $\left(1 \times 10^{3} \mathrm{kDa}\right)$ serine protease called extracellular proteasome (EC) and a group of metalloproteinases inhibited by TIMP-2. The purpose of these experiments was to assess the influence of Coumadin, an effective inhibitor of $\mathrm{EC}$ on in vitro and in vivo growth of $\mathrm{C} 6$ astrocytoma in the spheroid implantation model.

Methods: C6 and U251 cells were cultured in vitro with $10^{-5}$ and $10^{-6}$ concentrations of Coumadin. The effects on in vivo growth were assessed by giving coumadin to animals by p. 0 . and I.P. routes.

Results: Coumadin given I.P. at $0.25 \mathrm{mg} / \mathrm{kg}$ body weight on days 9,11 and 13 along with coumadin given by mouth after day $7(3.0 \mathrm{mg} / \mathrm{l}$ of drinking water $)$ resulted in significant decreased tumor weight at day 15 (control $121 \pm 13 \mathrm{mg}$ vs. 43.6 $\pm 6.7 \mathrm{mg}$ and $32.3 \pm 9.1 \mathrm{mg}$ respectively).

Conclusions: Coumadin given at the concentrations used had no influence on C6 or U251 monolayer growth but significantly decreased growth in vivo possibly through its inhibition of EC released by tumor cells.

P48.

Influence of Dexamethasone on Blood-Brain Transfer Constant and Plasma Volume of Brain Tumors

R.F. DEL MAESTRO, W.T.I. YEUNG, T.-Y. LEE, R. KOZAK, J. BENNETT and T. BROWN (London, Ontario)

Background: The symptoms of brain tumor-associated edema frequently responds to dexamethasone. These studies 
were carried out to assess the tumor blood-brain transfer constant of iopamidol $(\mathrm{K})$ and plasma volume $(\mathrm{Vp})$ of cerebral tumors before and after dexamethasone treatment.

Methods: Ten patients with primary brain tumors were treated with dexamethasone ( $4 \mathrm{mg}$ po qid) for 7 days and $\mathrm{K}$ and $\mathrm{Vp}$ assessed by X-ray computer tomography.

Results: Both $\mathrm{K}$ and $\mathrm{Vp}$ decreased significantly after treatment. The mean percentage decrease in $K$ was $34 \%(p<0.01)$ and $11 \%$ in $\mathrm{Vp}(\mathrm{p}<0.05)$. Functional images of these parameters clearly define the steroid effect on $\mathrm{K}$ while standard CT scans showed no difference in $80 \%$ of patients.

Conclusions: 1) The X-ray computed tomography method we have developed can be used to measure $K$ and $V p$ response of brain tumors to steroid therapy; 2) Dexamethasone significantly reduces $\mathrm{K}$ and $\mathrm{Vp}$ to small hydrophilic molecules and this may have chemotherapeutic implications.

\section{P49.}

\section{Central Nervous System Melanoma in Children}

\section{J.H. SAMPSON, J.H. CARTER, A.H. FRIEDMAN and H.F. SEIGLER (Durham, U.S.A.)}

Central Nervous System (CNS) metastatic disease is rare in children. The large number of patients with melanoma seen at our institution, however, allowed us to evaluate the demographics, risk factors, and prognosis of children with CNS metastatic melanoma.

From 1970 to $1990,6,953$ patients with melanoma were seen at our institution. A retrospective review identified 72 patients less than 18 years of age. CNS metastases were documented in 10 of these patients. Complete follow-up was available for all cases.

In these patients with CNS disease the average age at diagnosis was $16.3 \pm 1.2$ years. The male to female ratio was equal and all patients were Caucasian. The average delay from diagnosis of primary melanoma to CNS metastasis was 3.7 years. No patients had CNS disease at the time of primary diagnosis. Subsequently, however, 5 patients developed solitary and 5 multiple CNS metastases. Ulceration and increased depth of invasion tended to predict the development of CNS disease.

Mean survival from diagnosis of primary melanoma and CNS metastases was $4.1 \pm 2.9$ years and $160.7 \pm 123.4$ days respectively. The percentage of these patients alive and without clinical or radiographic evidence of CNS disease at $1,5,10$ years was $99 \%, 90 \%$ and $85 \%$. Cox-Mantel analysis of actuarial life tables demonstrated no significant difference in these survival characteristics relative to adult controls $(p>0.10)$.

In conclusion, although melanoma is rare in patients less than 18 years of age, these data, comprising the largest series to date, demonstrate no significant difference from adults in their manifestation of CNS metastases.
P50.

\section{Complications of First Craniotomy for Intra-Axial Brain Tumour}

\section{A. CABANTOG and M. BERNSTEIN (Toronto, Ontario)}

Complications were examined in a single surgeon's series of 181 consecutive adult patients undergoing craniotomies for intra-axial brain tumour. Excluded from the study were all repeat crainiotomies and all first crainiotomies for extra-axial tumour (e.g., meningioma). The study group consisted of 102 gliomas, 63 metastatic tumours, and 16 miscellaneous lesions. Gross total resection was performed for every metastatic tumour; for gliomas subtotal resection was performed in most cases.

All complications occurring within 30 days of surgery were identified. There were 5 deaths for a mortality rate of $2.8 \%$. The total number of patients sustaining complications was 48 for an overall complication rate of $26.5 \%$. Complications were divided into medical (e.g., deep vein thrombosis), wound (e.g., frontalis nerve palsy), seizures, intra-operative, hematoma, and neurological. Neurological complications were subclassified into transient neurological worsening, and permanent neurological worsening. Fifteen patients sustained transient neurological worsening apparently due to edema $(8.3 \%)$ and 6 patients sustained permanent neurological deficit (3.3\%). Medical complications were suffered by 13 patients (7.2\%). Major complications which significantly altered the quality and/or quantity of survival were incurred by 10 patients $(5.5 \%)$.

The complication rate for patients undergoing first craniotomy for glioma or metastasis has not been extensively examined and reported, and may be higher than is generally perceived.

P51.

\section{Brachytherapy for Recurrent Brain Metastasis}

\section{BERNSTEIN, A. CABANTOG, N. LAPERRIERE and C. THOMASON (Toronto, Ontario)}

Of 107 stereotactic high-activaty iodine-125 implants for malignant brian tumours since 1986 , ten were for solitary recurrent metastatic brain tumour and constitute the study group herein.

All patients had initially undergone crainiotomy for tumour resection followed by fractionated external beam whole brain radiation and recurred at the same site in brain. The interval between initial cancer therapy and occurrence of brain metastasis was 3 - 36 months (median 15 months). The interval between initial treatment of the brain metastasis and recurrence treated with brachytherapy was 3 - 16 months (median 8.5 months). Minimum brachytherapy dose was $70 \mathrm{~Gy}$ at a median dose rate of $67 \mathrm{cGy} /$ hour.

Five patients have died. Two died suddenly at two weeks and 13 weeks post implant of presumed pulmonary embolus. Three others died of brain recurrence at 20,39 , and 143 weeks post implant. Five patients are alive and recurrence-free at $31,35,57$, 
107 and 230 weeks post implant although one of these patients has developed systematic metastases. Two patients underwent reoperation for radiation necrosis (one is alive at 107 weeks and one died at 143 weeks). Two patients implanted lesions in the motor cortex incurred increase of motor deficit.

Brachytherapy appears effective in some highly selected patients with recurrent solitary brain metastasis. However the true efficacy of this modality is difficult to ascertain from small series such as this one, and randomized studies comparing brachytherapy to other modalities are needed.

\section{P52.}

\section{Colloid Cysts in Children}

\section{R.L. MACDONALD, R.P. HUMPHREYS, J.T. RUTKA and} J.R.W. KESTLE (Toronto, Ontario)

Three patients with third ventricular colloid cysts were identified amoung 1,400 patients with brain tumours at the Hospital for Sick Ckildren in Toronto between 1952 and 1992. Patients were 8,13 , and 15-years-old at diagnosis and 2 were female. Each presented with headache, vomiting, and papilloedema, following minor head injury in 2 cases. In one case, computed tomography (CT) and cerebral angiography did not show a cyst when he was 4-years-old. Four years later, however, CT showed the lesion which was removed transcallosally. Symptoms recurred 5 years later and a magnetic resonance imagining (MRI) scan showed recurrent colloid cyst. It was again successfully removed transcallosally. The second patient had a CT scan without contrast done for headaches after the head trauma which showed hydrocephalus but not the isodense colloid cyst. She suddenly became comatose, died despite emergent ventricular drainage, and the diagnosis was made at autopsy. In the third patient, enhanced CT and MRI showed a colloid cyst which was removed transcallosally. Colloid cysts are rare causes of headaches and papilloedema in children. The literature, containing only 34 cases of colloid cysts in children, is reviewed. Unless a CT scan with contrast or a MRI is done, the diagnosis may be missed, with fatal consequences. This report also shows colloid cysts can grow and sometimes recur post-operatively.

\section{P53. Withdrawn}

\section{P54.}

\section{Brain Tumor Patient Information}

\section{R.F. DEL MAESTRO (London, Ontario)}

The complexity of treatment options available for brain tumor patients has increased significantly in the last decade. Stereotactic radiosurgery, brachiotherapy, surgery under neuroleptic anesthesia and a variety of chemotherapeutic regimes are all being used. The patient and their family may be asked to make rather complex decisions based on information imparted to them by a variety of physicians. The results of controlled trials are not always available to help guide patient choices. To adequately inform the patients of their options, the risks and benefits of each option should be the goal of the physician patient contact.

The Brain Tumor Foundation of Canada has developed a Brain Tumor Patient Resource Handbook which optimizes the ability of patients to obtain the information needed. An NCR form (carbonless carbon) along with a number of drawings are available for the physician to write information. The physician keeps a copy of all the imparted information for his records which may decrease medicolegal concerns. This format may be useful for a variety of different surgical and medical procedures.

P55.

\section{Malignant Melanoma of the Scalp}

\section{J.H. SAMPSON, A.H. FRIEDMAN, and H.F. SEIGLER (Durham, U.S.A.)}

Neurosurgeons are often asked to assist in the management of patients with melanoma of the scalp which may involve the underlying skull and meninges. Still, little is known about the demographics, prognosis, and treatment results of these patients.

From 1970 to $1991,7,657$ patients with melanoma were seen at our institution. Primary melanoma lesions of the scalp were identified in 313 patients. In these patients, the average age at diagnosis was $50.0 \pm 15.4$ years. The male to female ratio was $3.6: 1$ and $99 \%$ of the patients were Caucasian. Follow-up time ranged from 15 days to 17.2 years with a mean of 4.7 years.

Male sex, deeper Clark's levels, and uncommon histologies distinguished patients with scalp melanoma from patients in our series with other primary sites. Although patients with scalp lesions were more likely to have tunor involvement of regional lymph nodes $(57 / 313)$ at diagonosis, they were less likely to have involvement of distant organs $(5 / 313)(p<0.001)$.

Median survival in 64 patients treated by local excision alone was 4.7 years. Actuarial life table analysis demonstrated a survival advantage for the 129 patients who underwent adjuvant immunotherapy with repeated subcutaneous injections of $2.5 \times$ $10^{7} \mathrm{X}$-irradiated melanoma tumor cells after local excision $(\mathrm{p}<$ 0.001 ). In those patients, median survival was 11.6 years. Patients who also underwent resection of regional lymph nodes had a worse prognosis probably indicative of more advanced disease $(p<0.001)$.

In conclusion, scalp melanoma is a distinct entity that will confront the neurosurgeon. The prognosis is generally poor, though the majority of patients present with locally limited disease. Finally, in this retrospective study, adjunctive immunotherapy was associated with an increased survival time. 
P56.

\section{L'Hermitte-Duclos Disease Mimicking Adult Onset Aqueductal Stenosis}

\section{S.N. SIDDIQI (North York, Ontario)}

L'hermitte-Duclos disease is a rare lesion characterized by enlarged cerebellar folia containing abnormal ganglion cells. This case report describes a 51-year-old female who was initially misdiagnosed with adult onset aqueductal stenosis, had a negative CAT scan, and was later found to have a midline cerebellar lesion extending to the brainstem by MRI. This is a unique case of L'hermitte-Duclos disease arising within the cerebellar vermis. The characteristic feature of an enlarged cerebellar hemisphere is absent on CT scan and MRI is needed to make the diagnosis. If diagnosed late, this "benign" lesion becomes difficult to resect totally and carries a poorer prognosis. Only two reports have mentioned MRI characteristics of L'hermitteDuclos disease. Both have been limited to T-2 weighted images. This case illustrates the full spectrum of MRI features for this disease. Both T-1 and T-2 weighted images show enlarged cerebellar folia within the lesion. The T-1 weighted image shows a mixed iso- and hypo-dense signal, the T-2 weighted image shows a homogeneously increased signal, and with gadolinium the lesion does not enhance. The latter feature supports the theory that this disease is a hamartoma rather than a tumor.

\section{P57.}

Temporal Lobe Epilepsy (TLE) Caused by Domoic Acid Intoxication: Evidence for Glutamate Receptor-Mediated Excitotoxicity in Man

F. CENDES, F. ANDERMANN, S. CARPENTER, A. EVANS, A. GJEDDE, J. TEITELBAUM, R. ZATORRE and N.R. CASHMAN (Montreal, Quebec)

In 1987, an outbreak of intoxication caused by contaminated mussels was documented in Canada. The mussel toxin was identified as domoic acid, an excitotoxin analogous to kainic acid.

We describe the development of TLE in an 84-year-old man, caused by domoic acid intoxication. The similarity with the kainic acid animal model of TLE was striking. The patient had nausea, vomiting and confusion on the day following the intoxication. He progressively developed coma, generalized convulsions and focal status epilepticus. After 3 weeks he improved but had severe residual memory deficit. EEGs initially showed periodic epileptic discharges, with left-sided predominance; later epileptic abnormalities over fronto-temporal regions and a diffuse slowing of the background developed. He had no further seizures and the EEG was normal 8 months after the intoxication. One year following the acute episode he developed complex partial seizures. EEGs showed epileptic discharges over both temporal lobes. MRI revealed hyperintense T2 signal and atrophy of the hippocampi. PET scan showed decreased bitemporal glucose metabolism. He developed pneumonia and died $3^{1 / 4}$ years after the intoxication. Autopsy disclosed severe bilat- eral mesial temporal sclerosis, but no other lesions usually found in post-anoxic state.

The development of TLE and hippocampal sclerosis following an acute illness caused by domoic acid ingestion, provides evidence for glutamate receptor-mediated excitotoxicity in man.

P58.

The Value of Performing Immediate Post-Ictal Clinical Examination of Patients with Complex Partial Seizures

\section{A.O. OGUNYEMI and H. BREEN (St. John's, Newfoundland)}

Post-ictal hemiplegia or Todd's paresis, occurring in some patients with symptomatic (lesional) partial seizures, is a well recognized phenomenon. Little attention has been paid to the presence of more subtle post-ictal signs in patients with partial epilepsy.

We performed selected clinical neurological examinations on 18 patients with epilepsy during 34 complex partial seizures. They were tested for: sensorium, language, left-right orientation, calculating ability, stereognosis and motor functions. Everyone of them had a CT scan of the brain and multiple EEG recordings. Sixteen had long term EEG and 8 had an MRI scan of the brain. Eleven patients had transient, lateralized, post-ictal neurological deficits. Language disturbance was present in 3 , visuospatial disorientation in 6, astereognosis in 4 and motor paresis in 4 . Only one of these patients had the post-ictal deficit for longer than 5 minutes. The neurological findings correlated very well with the results of the EEG and neuro-imaging tests.

P59.

Intensive Study of a Patient with Seizures Induced by Music A.O. OGUNYEMI and H. BREEN (St. John's, Newfoundland)

Musicogenic epilepsy is a rare disorder. Much remains to be learned about the electro-clinical features. This report describes a patient with seizures induced by music, who was studied with long-term telemetered EEG recordings.

This 27-year-old woman began to have seizures at the age of 10 years. She experienced complex partial seizures, often preceded by psychic symptoms in relation to singing, listening to music or thinking about music. She also had occasional grand mal seizures.

There was no significant antecedent history. Family history was negative for epilepsy. The general medical and neurological examinations were normal. CT amd MRI scans of the brain were normal.

During long-term EEG recordings, clinical and electrographic seizure activities were recorded in association with (i) singing alone, (ii) singing with friends and (iii) listening to certain songs, especially "Happy Bithday to You." One episode of nocturnal generalized convulsion was recorded. Calculation, copying or viewing geometric patterns and playing the game of chess, failed to evoke seizures. 
P60.

\author{
Cerebral Hemiatrophy Following Epilepsy Partialis \\ Continua in an Adult
}

\section{J.M. MIYASAKI and J. BILBAO (Toronto, Ontario)}

Cerebral hemiatrophy is not uncommon in the pediatric population secondary to encephalitis, perinatal asphyxia and preceding infection. Seizures commonly occur following the appearance of cerebral hemiatrophy. The modulators of brain damage caused by seizures are excitatory amino acids (EEA). Their site of action has been determined to be N-methyl-D-aspartate (NMDA) receptors. Previous authors have described characteristic patterns of neuronal necrosis following status epilepticus. These include necrosis in layers 3 and 4 of cerebral cortex primarily. Layer 2 is less commonly involved and layers 5 and 6 are rarely necrotic. All lobes are affected although the superolateral convexity appears to be most affected. These pattems have been described in animals. We present a case of cerebral hemiatrophy acquired in later life following intractable focal seizures. At autopsy, neuronal necrosis in a distribution corresponding to NMDA receptors was found. We propose that in some patients, seizures may be the underlying cause rather than a secondary manifestation of cerebral hemiatrophy.

P61.

Seizures Associated with Phenobarbital Withdrawal from Anti-Epileptic Drug Regimens: A Study of Institutionalized Patients.

\section{R.T. SEMMLER and D.F. WEAVER (Kingston, Ontario)}

Cognitive side effects of barbiturates are well described. Outpatient studies suggest barbiturate withdrawal from antiepileptic drug regimens are not associated with withdrawal seizures if other anticonvulsants are also taken. Work on institutionalized patients suggested withdrawal seizures do occur with primidone but not phenobarbital during the first 6 months but not at follow-up $6-12$ months later. The purpose of this study is to determine if phenobarbital withdrawal from anti-epileptic drug regimens worsen seizure control 6 and 12 months after withdrawal is completed. Fifty-eight institutionalized epileptic patients on phenobarbital and other non-sedative anti-convulsants with at least 1 seizure during a 1 year base-line period were prospectively studied. Phenobarbital was gradually stopped over an average of 8.3 months. Non-sedative anticonvulsants were adjusted to achieve optimal seizure control. Six month seizure counts immediately after phenobarbital withdrawal (mean 22.6, $\mathrm{p}<0.005$ ) as well as $6-12$ months after withdrawal (mean $17.6, \mathrm{p}<0.05$ ) had significantly more seizures compared to a baseline period (mean 12.6). Contrary to previous studies, these results suggest that withdrawal of phenobarbital may lead to withdrawal seizures and loss of long term seizure control. Other anticonvulsants may need to be substituted to maintain seizure control.
P62.

Emergency Care in Patients with Seizures; The Usefulness or the Uselessness of "Routine" Blood Tests

M.W. JONES (Vancouver, British Columbia)

One hundred consecutive Vancouver General Hospital Emergency visits were reviewed involving adults presenting with a seizure(s). A final diagnosis of epilepsy was made in 50 , alcohol and/or drug abuse in 37. For 10 it was their first seizure. Eighty arrived by ambulance and 17 were referred to neurology or neurosurgery (2).

Routine $\mathrm{CBC}$, electrolytes, BUN, CR, Mg, Ca and antiepileptic drug levels (AED) were usually measured. In 71 cases a CBC was done and in no case was it helpful. Electrolytes were measured in 69. The two lowest Na's were 125 and 127; both attributed to long standing Tegretol administration. Most of the low Na's (17 of 69) were not significant in the 130 to 134 range. In only 12 of 23 cases was the $\mathrm{Ca}$ indicated and in all was normal. For $\mathrm{Mg}, 11$ of the 22 measurements were indicated. For the 7 low $\mathrm{Mg}$ (mostly in alcoholics) nothing therapeutically was done about. AED levels were ordered in 46 cases and yet in 26 of these, the individuals had admitted non-compliance beforehand.

From an arbitrary point of view, potential savings of (CBC $\$ 15.11 \times 71=\$ 1072.81)+($ Lytes $\$ 20.07 \times 69=\$ 1384.83)+$ $(\mathrm{Mg} \$ 30.81 \times 11=\$ 338.91)+(\mathrm{Ca} \$ 14.81 \times 11=162.91)+$ AED $\$ 31.51 \times 26=\$ 819.26)=\$ 3778.72$ could have been achieved with more discretionary thought given to the ordering of these tests rather than ordering them "routinely".

P63.

Sudden Unexpected Death (SUD) in Epilepsy; A Review of 8 Cases

M.W. JONES (Vancouver, British Columbia)

Apparently normal epileptic individuals can suddenly and unexpectedly die without apparent demonstrable pathological cause once status, accidents, drowning, overdoses, intercurrent diseases, suicide, and other demonstrable causes have been ruled out. ("Epilepsy and Sudden Death" by Lathers and Schraeder-1990 Marcel Dekker, Inc. Publishers). Autopsies typically show no significant abnormality that would explain death. This phenomena continues to be an enigma despite its relatively common occurrence accounting for approximately $15 \%$ of all deaths in persons with epilepsy. It may be as high as 1 in 50 in males between the age of 20 to 40 with symptomatic epilepsy who are non-compliant and have structural brain disease.

Eight patients "found down dead" unexpectedly had Coroners autopsies and no pathological explanation was found. Seven were men. Age ranged between 22 to 69 with 4 of the 8 in their 20's. The patient with $1^{\circ}$ generalized epilepsy had been seizure free for four years whereas all the rest were poorly controlled. Two had the Lennox-Gastaut Syndrome, 3 had CPS of whom 2 had unsuccessful surgery and in only 1 was there therapeutic AED's. 
This small series parallels the generalizations made above. SUD most likely to occur in men with poorly controlled seizures who have had it for decades, regrettably no new insights were gleaned as to the mechanism which is assumed to be on a cardiac arrhythmic basis.

\section{P64.}

\section{Hyperglycemia Presenting as Intractable seizures}

\section{S. WHITING, A.E. MACKENZIE and P. HUMPHREYS (Ottawa, Ontario)}

The occurrence of focal epilepsy as a presenting feature of hyperglycemia without ketosis has been well recognized. However, this phenomenon has been mainly documented in adults after the third decade; pediatric experience has been relatively rare.

We report two teenage girls with long standing epilepsy but no prior history of diabetes, one of whom had had a temporal lobectomy three years prior to presentation. The other patient had Sturge Weber Syndrome with well controlled seizures. Both presented with intractable focal motor seizures unresponsive to anticonvulsants including Dilantin.

In both cases, blood glucose levels were done after several days of hospitalization when mild polyuria and polydipsia were noted. Glucose levels were high $(20 \mu \mathrm{mol} / \mathrm{L}, 29 \mu \mathrm{mol} / \mathrm{L})$ with mild ketosis and acidosis in the first patient. With institution of insulin, there was prompt cessation of seizures. The patients were diagnosed as having Type I insulin dependent diabetes mellitus and require ongoing insulin and also continue on anticonvulsants.

Hyperglycemia should be considered in patients who present with intractable epilepsy.

\section{P65.}

\section{Inheritance Patterns in Familial Epilepsy}

\section{J. MAHER and R.S. McLACHLAN (London, Ontario)}

In preparation for an epilepsy gene mapping study, we have identified six large families with multiple members affected by seizures. Seizures occurred in 5 - 10 (mean 8) of $20-42$ (mean 31) family members across two to five generations giving a prevalence of $25 \%$. The phenotypic expression was generalized seizures of various types (grand mal seizures with or without mental retardation, grand mal seizures upon awakening, febrile convulsions) but four patients subsequently developed complex partial seizures. Penetrance was age dependent. No consistent pattern of inheritance was seen in the various families. There was no consanguinity but combined paternal and maternal inheritance occurred in two families. Both genotypic and phenotypic heterogeneity must be taken into account in any search for a seizure susceptibility gene.
P66.

\section{Barbiturate Anesthesia is the Treatment of Choice for Refractory Status Epilepticus}

F. VELOSO ( Regina, Saskatchewan)

Status epilepticus is a neurologic emergency. There is general agreement that I.V. Diazepam and/or Phenytoin is the drug of choice in the treatment of status epilepticus. There is less consensus regarding the next line of treatment if seizure persists despite I.V. Diazepam and Phenytoin. We reviewed eight patients with nine discreet episodes of refractory status epilepticus that we treated successfully with barbiturate anesthesia using Pentobarbital or Pentothal. Based on our experience, we recommend that barbiturate anesthesia should be the treatment of choice for refractory status epilepticus. We will propose a protocol for the use of I.V. Pentothal in the treatment of refractory status epilepticus that can be safely carried out even in primary care institutions without monitoring capabilities.

P67.

Traumatic Intracranial Hematoma Due to Seizures: Identification of a High-Risk Patient Group

\section{T. ZWIMPFER, R. MOULTON and I. SULLIVAN (Toronto, Ontario)}

Data were collected prospectively on 1259 consecutive patients admitted to hospital with head injury. Injury resulted from a seizure in 16 patients $(1.3 \%)$. This number falls within estimated prevalence rates of epilepsy. The mean age of the patients with seizures (45), GCS (9), and ISS (21) were similar to those in the non-seizure group $(41,10$, and 25 respectively).

All 16 patients with seizures fell and had intracranial hemorrhages on CT scan. Fifteen $(95 \%)$ required operative management. Operable mass lesions were significantly more common in the seizure group than in all patients without seizures $(95 \%$ vs. $25 \%, \mathrm{p}<0.001, \chi^{2}=38.2$ ), and in patients who fell without seizures $\left(95 \%\right.$ vs. $42 \%, \mathrm{p}<0.001, \chi^{2}=16.8$ ). Head injury due to a seizure was not associated with greater mortality.

While we could not show that patients with seizures have an increased risk of head injury, those patients who sustain a head injury as a result of a seizure have a markedly increased risk of operable intracranial hematoma. Injury in a fall is known to carry an increased risk of traumatic intracranial hematoma. The absence of protective responses due to unconciousness may account for the further increase in risk of intracranial hematoma in seizure patients. All patients injured in a fall due to a seizure should have a CT scan. 
P68.

\section{Focal Seizures in Non-Ketotic Hyperglycemia}

J.E. SILVA and G.B. YOUNG (London, Ontario)

Two diabetics had focal seizures only during subacute nonketotic hyperglycemia.

A 57-year-old woman had jerks of the right upper limb and face. She then had nystagmus retractorius in which left hemisphere periodic lateralized epileptiform discharge preceded the ocular movements by $150 \mathrm{msec}$. Serum glucose was 50.5 $\mathrm{mmol} / \mathrm{L}$.

A 51-year-old male had various complex visual illusions and detached feelings. He also had 3 clonic seizures in the right parietal-occipital-posterior temporal region, where an HMPAOSPECT scan showed increased uptake. Serum glucose was 24.4 $\mathrm{mmol} / \mathrm{L}$.

CT of brain, MRI of brain (2nd patient), serum osmolality and serum and urine ketones were normal in both patients.

Our and previously published cases share the following: subacute, non-ketotic hyperglycemia; focal seizures which resolved with correction of hyperglycemia; no recurrence, normal neuroimaging of structures.

We propose hyperglycemia-associated seizures arise from a biochemical rather than vascular mechanism.

\section{P69.}

The Importance of Automated EEG Monitoring in Defining Chronic Epilepsy Models

\section{C.R. MASCOTT, J. GOTMAN and A. BEAUDET (Montreal,} Quebec)

There has been a recent surge of interest in chronic animal models of epilepsy. Documentation of spontaneous seizures by EEG, observation, or both should be performed for each individual animal to confirm the presumed epileptic condition regardless of the model used. This can be quite difficult since, unlike provoked seizures, spontaneous seizures usually occur infrequently and unpredictably. We have employed the same automatic seizure detection system as that used currently for patients in this and many other institutions. Fifty-four rats were implanted with electrodes prior to intra-amygdalar administration of kainic acid (KA). Of these, 43 were monitored intermittently over a 3 month period. Spontaneous seizures were detected in $18.6 \%$ of rats at 1 to 2 months after $\mathrm{KA}$ injection and in $44.2 \%$ after 2 to 3 months. A total of 22 of the 43 rats $(51 \%)$ had a confirmed spontaneous seizure between 1 and 3 months after KA injection. A majority of the remaining rats showed some electrographic epileptiform changes but actual seizures were not recorded. We caution that it is impossible to exclude the presence of seizures in any rat (or patient). On the other hand, definitive proof of epilepsy is invaluable when trying to interpret data from rat brains.
P70.

\section{Hemispherotomy: The Peri-Sylvian Approach}

\section{J.-G. VILLEMURE and C. MASCOTT (Montreal, Quebec)}

Anatomical hemispherectomy for the control of medically refractory seizures has been replaced by the "modified hemispherectomy", the "hemidecortication" and the "functional hemispherectomy". Functional hemispherectomy consists in a disconnective procedure with limited brain removal. The extent of brain removal is guided by the degree of ventricular enlargement which may vary according to the etiology of the disease or the timing of the operation in relation to the onset of the disease. The surgical procedure with the smallest brain removal to achieve a complete hemisphere disconnection should be termed "hemispherotomy". When a functional hemispherectomy is indicated, hemispherotomy should be considered when the whole ventricular system is very large. The procedure is carried out by disconnecting the whole hemisphere through a supra and an infra-sylvian approach giving access to the ventricle which allows the disconnection. The only structure that needs to be removed is the amygdala. We describe the surgical technique and report preliminary experience.

P71.

\section{Epilepsy and the World of Shakespeare}

\section{J.A.R. TIBBLES (Victoria, British Columbia)}

William Shakespeare describes epileptic seizures in four of his main characters with remarkable clarity.

1. Henry IV (1598) - generalized epilepsy

2. Julius Caesar (1600) - generalized epilepsy

3. Othello (1604) - complex partial seizure

4. Macbeth (1605) - brief probable complex partial seizures.

The physician, John Hall, was only 10 years younger than Shakespeare. He arrived in Stratford in 1600 and married the playwright's daughter seven years later. His possible role in the later seizure descriptions is discussed.

Hall recorded "select observations" on his treatment of "desperate diseases of eminent persons". This treatise contains descriptions of three cases of epilepsy and four of hysterical seizures. Treatments included peony, both taken internally and worn as an amulet, mistletoe, Venice treacle and pulverised men's skull, which were in keeping with contemporary management as well as conserve of scurvy grass for scorbutic epilepsy. A high incidence of hysterical disease is found in Hall's series and some manifestations of Elizabethan hystero-epilepsy are reviewed. 
P72.

Segmental Symptoms in Rats Associated with Chronic Intrathecal Infusion of NMDA: Evidence for Selective Action in the Dorsal Horn

\section{D.W. ZOCHODNE, M. MURRAY, S. NAG and R. RIOPELLE (Calgary, Alberta; Kingston; Toronto, Ontario)}

Single intrathecal injections of NMDA (n-methyl, d-aspartate) into mice activate nociceptive pathways in the spinal cord dorsal horn. Previous work (SN and RR) identified excitotoxic necrosis of anterior and posterior horn cells following chronic intrathecal infusion of NMDA. We explored the influence of chronic lumbar intrathecal infusions (by mini-osmotic pumps) of NMDA in Sprague-Dawley rats on motor and sensory axonal integrity as assessed by multifiber serial in vivo and endpoint in vitro motor and sensory conduction measurements. The following infusion protocols were employed: (i) $0.15 \mathrm{M}$ NMDA in PBS (phosphate buffered saline), 4 weeks, $n=10$; (ii) PBS without NMDA, 4 weeks, $\mathrm{n}=11$; (iii) $0.20 \mathrm{M}$ magnesium sulfate $+0.15 \mathrm{M}$ NMDA, 4 weeks, $\mathrm{n}=7$; (iv) $0.35 \mathrm{M}$ NMDA in PBS, 4 weeks, $n=3$; (v) 0.15 M NMDA in PBS, 8 weeks, $n=9$; (vi) PBS without NMDA, 8 weeks, $n=8$.

Within $1-2$ weeks of the onset of NMDA, but not PBS infusions, the rats exhibited irritability, circling, self-biting and excessive grooming of the hindquarters with loss of hair and ulcerations from autotomy. The self injury was localized to the lumbar and sacral segments appropriate to the level of infusion. Co-infusion of NMDA with magnesium sulfate prevented autotomy in all but one rat. In vitro and in vivo conduction studies identified preservation of myelinated motor, myelinated sensory and unmyelinated sensory axonal integrity in NMDA treated animals.

Intrathecal NMDA induces a chronic "central" experimental pain disorder in rats without involvement of peripheral sensory pathways and that spares axons of anterior horn cells.

\section{P73.}

$\mathrm{N}$ of 1 Randomized Controlled Trial of Ketotifen to Control Symptoms from a Massive Plexiform Neurofibroma in a Patient with Neurofibromatosis

\section{D.J. BUCKLEY, K. GORDON, J. DOOLEY and P. CAM-} FIELD (Halifax, Nova Scotia)

Ketotifen is a powerful $\mathrm{H} 1$ receptor blocking agent, proposed as useful in treatment of Neurofibromatosis Type 1 for pruritis, pain and tenderness. (Possibly due to the release of histamine from many mast cells associated with neurofibromas).

No controlled studies have been reported on Ketotifen use in Neurofibromatosis.

We performed an $\mathrm{N}$ of 1 study to assess the usefulness of Ketotifen in a teenage boy with Neurofibromatosis Type $1 \mathrm{com}-$ plicated by a large plexiform neurofibroma of his right leg and significant somatesthetic symptoms.

He was given, on a monthly basis, either Ketotifen or placebo for ten months. At the end of each month he answered a ques- tionnaire regarding his symptoms and volumetric measurements were taken of both legs.

Results failed to show any improvement in somatesthetic symptoms of his leg. During the study the patient lost $8.65 \mathrm{Kg}$ for unknown reasons. Reduction of volume was the same in both legs.

We conclude that Ketotifen did not help our patient. Our experience casts doubt on the value of Ketotifen in Neurofibromatosis Type 1 and emphasizes the value of $\mathrm{N}$ of I trials.

P74.

\section{SPECT Scanning in Motor Neurone Disease}

R.K. MOSEWICH, J.R. DONAT, C.L. VOLL, C.J. CHMIELOWIEC and A.A. WILKINSON (Saskatoon, Saskatchewan)

There is no widely available radiological test useful in the diagnosis of motor neurone disease (MND). Positron emission tomography (PET) scanning has demonstrated widespread cortical hypometabolism in MND. The role of single photon emission computed tomography (SPECT) in MND is not well studied. We report the results of $99 \mathrm{mTc}$ HmPAO SPECT scanning performed on 11 patients with motor neurone disease.

Of the 11 patients studied, 9 were male and 2 were female. The average age was 60 years and the average duration of symptoms was 1.9 years. Clinically, 9 patients had evidence of lower motor neurone disease and 7 had definite evidence of upper motor neurone disease. Four patients had preserved reflexes in the presence of significant atrophy. Three patients had a pseudobulbar palsy and 6 had a bulbar palsy.

The SPECT scan was abnormal in $6(55.5 \%)$ patients. The abnormality consisted of varying degrees of bifrontal hypoperfusion which in cases extended both anteriorly and posteriorly. The results suggest that SPECT scan is not highly sensitive in the diagnosis of MND. There may however be a role for SPECT in providing supplementary evidence in diagnostically difficult MND cases.

P75.

Fatigue Persists for at Least 1 Hour after Low-Intensity Forearm Exercise: Lack of Correlation with Lactate or Blood Flow

\section{V.A. CWIK, K.J. HUTCHISON, R. MAJUMDAR and M.H. BROOKE (Edmonton, Alberta)}

Many patients experience muscle fatigue. Forearm exercise tests (FET) fail to demonstrate abnormalities during the exercise phase in the majority. The phenomenon of recovery is less well investigated. Normal data is required before patients can be evaluated. We investigated muscle fatigue during and following submaximal exercise. We demonstrate that lack of recovery is identical at 1 hour following both low- and high-intensity exercise and is not due to lactate accumulation or blood flow.

Twenty-two volunteers performed intermittent FET ( 2 seconds contraction, 1 second relaxation) at $20 \%$ or $40 \%$ maximum 
voluntary contraction (MVC) for 10 minutes. Force, venous lactate and subclavian artery blood flow (SABF) by duplex ultrasound were measured during exercise and 1 hour of recovery.

During 40\% FET there is a steady decline in force, a 3.5 -fold rise in mean SABF and a $>4$-fold rise in lactate. Following exercise, SABF and lactate return to near baseline levels by 10 and 45 minutes respectively. $20 \%$ FET is associated with no fatigue and $\mathrm{a}<2$-fold rise in mean SABF and lactate during exercise. Immediately following $40 \%$ FET, grip strength is $68 \%$ of MVC; after $20 \%$ FET it is $83 \%$. One hour after exercise grip strength is the same but reduced in both $(85 \% \mathrm{MVC})$.

\section{P76.}

Comparison of Some Sensory and Motor Nerve Conduction Parameters in the Assessment of Carpal Tunnel Syndrome

\section{R.J.H.M. ARTS (St. John's, Newfoundland)}

No gold standard exists for the detection of carpal tunnel syndrome, therefore, sensitivity and specificity of the various employed electrodiagnostic tests cannot accurately be established. A comparison of the rate of abnormality of different neurophysiological parameters was performed in patients clinically suspected of having a Carpal Tunnel Syndrome.

Motor Parameters studied are: Distal Motor Latency (DML), Compound Muscle Action Potential (CMAP) Amplitude changes, and Motor Nerve Conduction Velocity over the carpal tunnel using proximal and distal stimulation. Sensory parameters include Sensory Nerve Action Potential (SNAP). When appropriate, left right comparison as well as comparison with responses using ulnar nerve stimulation was undertaken.

Clinical History and neurological examination were considered in defining the clinical entity.

Standard statistical measures were used in evaluating the results.

The results will be presented, their significance discussed.

P77.

Acute Fulminating Myasthenia Gravis: Misleading Electrophysiological Findings in One Patient During a Myasthenic Crisis

\section{F. GRAND'MAISON and L. LAMOUREUX (Sherbrooke, Quebec)}

EMG characteristics of acute fulminating myasthenia gravis (AFMG) include: normal amplitude of " $M$ " responses, decrement of the supramaximal " $M$ " response on repetitive stimulation (RS) and absence of abnormal spontaneous activity on needle examination. We describe a patient with AFMG in whom EMG findings were atypical of neuromuscular transmission defects and suggested alternative diagnoses.

A 38-year-old female patient, well known for seronegative MG, presented with a myasthenic crisis characterized by acute ventilatory failure and by rapidly progressive and fluctuating diplopia, dysphagia and dysphonia. Examination revealed primarily ocular, bulbar and respiratory muscle involvement.

Routine nerve conductions were normal. However, on phrenic nerve stimulation, diaphragmatic responses were markedly reduced in amplitude. A significant decremental response was not found in RS of the ulnar, accessory or facial nerves but was suspected on RS of the phrenic nerves. Needle examination revealed fibrillation potentials multifocally, particularly in the diaphragm.

It is concluded that EMG abnormalities may be misleading in acute fulminating myasthenia gravis and that these findings may be most apparent of phrenic nerve conduction studies and on needle examination of the diaphragm.

\section{P78.}

Phrenic Nerve Conduction in Chronic Obstructive Pulmonary Disease: Giant Diaphragmatic Potentials

\section{F. GRAND'MAISON and F. EVOY (Sherbrooke, Quebec)}

The role of phrenic nerve conduction studies to evaluate respiratory muscle function in patients with respiratory failure is increasingly recognized. A reduction in the amplitude of the diaphragmatic potential (DP) evoked by phrenic nerve stimulation strongly suggests dysfunction of the phrenic nerve or of the diaphragm. Since the DP amplitude in normals is considerably affected by changes in lung volume, we set out to determine the effect of the hyperinflated lung of patients with chronic obstructive pulmonary disease (COPD) on the DP.

Eleven patients with compensated COPD of variable severity and 14 normals were studied. The phrenic nerve was stimulated in the neck at functional residual capacity and the DP was recorded over the chest wall.

The average negative peak amplitude of the DP was $1234 \mu \mathrm{v}$ in COPD patients and $559 \mu \mathrm{v}$ in controls $(\mathrm{p}<0.001)$. The DP amplitude in COPD patients was highly correlated $(p<0.001)$ with residual volume, a marker of lung hyperinflation.

It is concluded that the amplitudes of the DPs are much larger in COPD patients than in healthy subjects. This effect is probably related to flattening of the diaphragm associated with lung hyperinflation. Results of phrenic nerve conductions in COPD patients must be interpreted accordingly.

P79.

Incidence of Distal Sensorimotor Polyneuropathy in Early HIV Infection

M. VEILLEUX, J. FALUTZ and O. PALTIEL (Montreal, Quebec)

Distal symmetric polyneuropathy (DSPN) has been reported in 20 to $75 \%$ of patients with AIDS particularly in advanced stages of the disease. Twenty patients infected with human immunodeficiency virus (HIV) were studied prospectively to determine the frequency of DSPN in clinical stage II of HIV 
infection and the role of vitamin B12 deficiency on the incidence of DSPN in HIV patients. All patients had serum vitamin B12 level, Schilling test, and electrodiagnostic studies including nerve conduction studies and concentric needle examination in the lower extremities, and sympathetic skin responses (SSR) of the hand and foot. Only 4 patients (20\%) had clinical or electrophysiological evidence of DSPN. Based upon clinical signs, one had a mild predominantly sensory DSPN and another complained of numbness in the hands and feet but had normal neurological exam. Of the 2 patients with abnormal electrodiagnostic studies, one had no response on sural, superficial peroneal and medial plantar nerve stimulation, and the other had no SSR in the foot. Of the 6 patients with abnormal Schilling test, only 1 patient had DSPN based on abnormal electrodiagnostic studies. Evidence for DSPN was present in $20 \%$ of patients with early HIV infection and did not appear to be more frequent in patients with concurrent vitamin $\mathrm{B} 12$ deficiency.

\section{P80.}

\section{Macrophage Reactions in Rat Dorsal Root Ganglia After Nerve Injury}

\section{LU and P.M. RICHARDSON (Montreal, Quebec)}

In a previous study, the adventitious recruitment of macrophages to the DRG (dorsal root ganglia) was found to mimic axotomy in stimulating regenerative responses in sensory neurons. In follow-up, immunohistochemical studies with three monoclonal antibodies were undertaken to investigate reactions of macrophages in rat DRG. Approximately 4000 cells with macrophage antigenicity were estimated to be present in normal lumbar DRG. The number of macrophages started to increase 2 - 4 days after sciatic nerve transsection, remained elevated for 4 weeks, and returned to normal by 8 weeks. Many of these macrophages were in perineuronal position and morphologically similar to satellite glial cells but were distinguished from satellite glial cells by their lack of immunoreactivity to S-100 antibodies. We speculate that these perineuronal macrophages are a source of trophic support to injured neurons.

\section{P81.}

\section{Reference Electrode Contribution to the Ulnar Compound} Motor Action Potential

\section{R.Z. KERN and A. ALEX (Mississauga, Ontario)}

The abductor digital minimi (ADM) compound motor action potential (CMAP) elicited by ulnar nerve stimulation is a summated surface potential to which the muscle (G1) and tendonreference (G2) electrodes make a significant contribution. A quantitative analysis of $\mathrm{G} 1$ and $\mathrm{G} 2$ potentials in 10 normal individuals was performed using the contralateral hand $(\mathrm{CH})$ as a reference.

G2-CH recordings demonstrated a triphasic far-field potential with a constant onset latency, however the initial negative peak increased and subsequent positive peak decreased moving from digit 5 to digit 2 . A more distal recording site on digit 5 did not influence the $\mathrm{G} 2-\mathrm{CH}$ potential. $\mathrm{G} 1-\mathrm{CH}$ recordings demonstrated a biphasic potential with a larger negative peak amplitude, shorter negative peak latency and duration, and smaller negative peak area than the conventional ADM CMAP.

In normal individuals the ADM CMAP can appear as a single or double-peaked potential depending on the relative amplitudes and phase relationships of the G1 and G2 potentials. Variations in Gl electrode placement also influence the waveform. These variations in CMAP waveform, amplitude and area should be considered in estimation of conduction block or other quantitative analysis.

P82.

\section{Migraine in Later Life}

J. RAVINDRAN, A. SHUAIB, and R. MOSEWICH (Saskatoon, Saskatchewan)

Onset of migraine is unusual after the age of 50 . The incidence of migraine in patients over 65 years of age is $12 \%$ in women and $7 \%$ in men. We report 13 patients who had migraine in later life. Mean age was 65.6 years with a range of 55-76. Onset of symptoms were $<1$ year in 9 patients. Four patients had onset from 3-15 years and developed migraine after variable periods without attacks. Eight had common migraine without aura. One patient had migraine with visual aura. Four had common migraine as well as episodes of migraine aura without headache. Two patients had left sided sensory symptoms with visual aura. One patient had unsteadiness, incoordination and visual aura. One patient had transient non-fluent aphasia. All four patients had normal CT scan, carotid doppler ultrasound and normal ESR. Four patients had infrequent migraine without aura and were treated with symptomatic analgesics, six with amitriptyline, two with flunarizine and one with propranolol.

Decreasing prevalence of migraine with age is believed to be due to changing vascular compliance and hormonal factor. Other causes of headache should be excluded in this age group before migraine is diagnosed. Migraine aura with transient focal neurologic symptoms should be recognized and distinguished from TIA. They respond to therapy as well as younger people.

P83.

\section{C.T. Evaluation of the Chronic Headache Patient}

\section{DUMAS and J.H.W. PEXMAN (London, Ontario)}

Objective: Are contrast enhanced C.T. head scans to detect tumour, aneurysm or AVM cost effective in patients with chronic headache?

Method: We reviewed 402 C.T. head scans of 373 consecutive patients referred from a chronic headache clinic. Patients had no neurological physical signs and their mean age was 39 (13 - 86). Females outnumbered males 3:1. Non ionic contrast medium was used. Microcosted technical fees were added to professional OHIP fees. 
Results: There were 14 (3.5\%) minor findings which did not alter management ( 9 infarcts, 2 cerebral atrophy, one cavum vergae, one hyperostosis frontalis interna, one communicating hydrocephalus) and $4(1 \%)$ significant lesions (2 osteomas, one low grade glioma, one aneurysm). Only the aneurysm was treated. There were no AVM's. All lesions except the aneurysm were seen without contrast. A non contrast scan costs $\$ 82.63$, an enhanced one $\$ 204.05$. It cost $\$ 75,243$ to find one treatable vascular lesion.

Conclusion: This does not appear to be a cost effective procedure. It could be replaced by reassurance that the incidence of a significant lesion approaches that found randomly in the general population. An unenhanced C.T. scan costing $40 \%$ of an enhanced C.T. scan showed all tumours.

\section{P84.}

Flunarizine in the Treatment of Migraine With and Without Aura

M. GAWEL, J. KREEFT, D. SIMARD and R. NELSON (Toronto; London, Ontario; Quebec City, Quebec; Ottawa, Ontario)

Flunarizine (FLU), a selective $\mathrm{Ca}^{++}$blocker, is effective in migraine prophylaxis. Previous trials have shown that improvement ranges from $50-80 \%$; however, these studies were usually of 3 months' duration. Currently, there is a paucity of data on the long-term effects (i.e. $>3$ months) of FLU. The objective of this study was to determine the long-term efficacy and safety of FLU. Patients aged $18-60$ years with 2 - 8 attacks of migraine per month were eligible. After completing a single-blind onemonth placebo run-in, 147 patients were treated with FLU 10 $\mathrm{mg}$ h.s. for 5 months. Patients were seen monthly. At each visit, the number of days per month with migraine, duration of each attack and number of days relief medications were used were recorded. Patients kept daily diaries to record severity of attacks and associated symptoms (e.g. photophobia). At the end of the run-in period, the mean number of days with migraine was 7.8 . This decreased during the course of therapy so that by trial end, the mean number of days with migraine was $3.6(p=0.0001)$. Over $50 \%$ of patients had at least a $50 \%$ reduction in days with migraines. At trial end, $16.5 \%$ were migraine-free. There was a significant reduction in the number of days relief medications were used (baseline $=6.5$, end of therapy $=3.0, p=0.0001$ ) and mean duration of migraines (baseline $=17.4 \mathrm{hrs}$, end of therapy $=9.1 \mathrm{hrs}, \mathrm{p}=0.0001$ ). Eighty-seven per cent of patients were responders to therapy. The most common side effects were weight gain $(30 \%)$ and fatigue (20\%). FLU had no effect on cardiovascular parameters. This trial confirms the efficacy and safety of FLU for the treatment of migraine. Currently, a subset of patients is being monitored after stopping flunarizine to determine when migraines recur.
P85.

Migraine Prophylaxis with Flunarizine: Effectiveness is Significantly Better at Six Months Compared to Three Months

K. BROWNE, A. SHUAIB, S. LICO (Saskatoon, Saskatchewan)

Patients with frequent migraines may benefit from prophylactic therapy. Most studies using prophylactic therapy have looked at the effectiveness of only 6 to 8 weeks. The purpose of this study was to compare the effectiveness at 3 and 6 months of therapy in a multi-centre Canadian trial where patients were followed every month for a total of 6 months after initiation of therapy.

A total of 137 patients with migraine were enrolled. The mean age was 38.2 years (male 39.8 years) and females (37.9 years). At onset, the number of migraines per month were 8.5 $( \pm 0.41)$, then it decreased to $5.2( \pm 0.37)$ at 3 months and 3.3 $( \pm 0.33)$ at 6 months. Compared to 3 months, the decline at 6 months was significantly better $(p<0.05)$. At the same time, the duration of episodes decreased from 9.3 hours $( \pm 0.42)$ at onset, to 8.75 hours $( \pm 0.5)$ at 3 months and 6.8 hours $( \pm 0.55)$ at 6 months. Again, the decline at 6 months compared to 3 months was significantly better. Other scores, including the severity of nausea, photophobia and sonophobia were also significantly better at 6 months compared to 3 months.

Flunarizine at a dose of $10 \mathrm{mg}$. is effective in decreasing migraine symptoms at 3 months, with further improvement in the subsequent 3 months. It is thus recommended that patients with migraine should have therapy for more than 3 months to adequately judge efficacy.

P86.

Prolonged Flunarizine Therapy is Associated with a Decline in the Use of Abortive Medications, Including Narcotics

K. BROWNE, A. SHUAIB, S. LICO (Saskatoon, Saskatchewan)

One major goal of migraine prophylaxis is to avoid frequent use of narcotic and non-narcotic therapy to abort headaches. The goal of this study was to look at the effects of Flunarizine on concomitant use of narcotic and non-narcotic medications to abort headaches.

A total of 137 patients with migraine were enrolled. Analysis at three months and at six months showed that ancillary medications were used for 6.5 days per month at onset, 4.6 days at three months and 3.0 days at six monhs. The total number of medications used per month declined from 21.2 at onset to 15.2 at three months and 9.2 at six months. There was a decline in the total number of non-narcotic tablets from 13.9 at onset to 9.9 at three months and 7.4 at six months. Importantly, a decline in the total number of narcotic medications was from 7.3 per month at onset to 6.2 at three months and 1.8 at six months. All scores were significantly better at six months when compared to three months. 
It appears that as the frequency of headaches decrease, severity may also decline. The decreasing use of narcotics by the patients on Flunarizine therapy is encouraging. This decreased use was significantly better at six months compared to three months. As migraine is a chronic disorder, the use of prophylactic medicines should be used early to avoid the potential for drug addiction.

\section{P87.}

\section{The Observations of Sir Gordon Holmes from World War I}

\section{R.M. SADLER, T.J. MURRAY and A.T. IRVINE (Halifax,} Nova Scotia; London, England)

Most neurologists are aware of Holme's contributions to neurology. Relatively little attention has been given to the circumstances under which Holmes made the observations that became the basis of some of his best known publications.

In 1914, Holmes was appointed as a consultant neurologist near "The Front" in France. By 1918 he had, while engaged in active medical care, published 17 papers on neurologic complications of warfare. Examples include observations on injuries of the superior longitudinal sinus, spinal cord (over 300 cases), cerebellum ( 70 cases), and several papers on cortical visual dysfunction. Holmes also designed a system to classify and treat the thousands of soldiers with psychological problems from battle.

The working conditions were extraordinary. Cushing Cushing described Holmes' hospital as having an attending staff of 10 - 16 caring for 900 critically ill patients and often receiving daily convoys of 300 acutely wounded soldiers.

It is remarkable that Holmes, in such circumstances, could care for patients, write papers, record abnormal movements on rotating smoked drums, perform detailed visual field assessments, urodynamic studies, and autopsies. The results of these investigations contributed major advances that still serve clinicians today.

P88.

\section{Dynamic Analysis of Motor Function After Functional Hemispherectomy}

S.E. BRIEN, J.G. VILLEMURE and T.B. HOSHIZAKI (Halifax, Nova Scotia; Montreal, Quebec)

Functional hemispherectomy consists of complete callosotomy, complete disconnections of frontal and parietal-occipital lobes in the coronal plane and a temporal lobectomy. Experienced epilepsy surgeons have noted not only improved control following this procedure but subtle improvement of motor function in their pre-surgical hemiplegia.

Recently researchers have used inverse dynamic solutions to divide movement patterns into functional phases for more accurate and objective quantification of motor function. A thirtyyear-old female with a chronic disorder was evaluated before and after undergoing a functional hemispherectomy using a high speed 3-dimensional video analysis and inverse dynamics.
The data revealed significant changes in the patient's joint torques and movement patterns before and after the procedure. The implications of the results will be discussed in terms of usefulness of the analysis technique and changes in patient motor function.

P89.

\section{Chiasmal Compression and Acute Ophthalmoplegia in Two Cases of Pituitary Metastasis}

\section{S.E. BRIEN and G. MOHR (Montreal, Quebec)}

Metastasis to the pituitary gland is a rare diagnosis that is often not made until autopsy despite the incidence of nearly twenty percent in those that succumb to systemic disease.

Two patients, one who developed acute loss of vision from chiasmal compression and another developed bilateral cavernous sinus syndrome from pituitary metastasis are presented. Both patients had improved vision and ocular motor function respectively after decompressive transphenoidal procedures and radiotherapy.

Visual recovery in cases of pituitary pathology as well as the pathophysiology of visual loss and hematogeneous spread to the pituitary gland and cavernous sinus will be discussed.

We conclude that pituitary metastasis is an important differential diagnosis in sellar and parasellar lesions, especially when there is evidence of posterior pituitary lobe dysfunction and that transphenoidal decompression followed by radiotherapy can allow satisfactory visual recovery.

P90.

Differentiating Pituitary Apoplexy and a Giant Intrasellar Aneurysm with Magnetic Resonance Imaging

\section{R.D. BROWNLEE, F.E. LEBLANC and R.J. SEVICK (Calgary, Alberta)}

A case is presented of a patient with a giant internal carotid artery aneurysm presenting as an intrasellar mass lesion.

A 64-year-old female was awakened from sleep by the sudden onset of a severe headache associated with nausea, vomiting and photophobia. On examination the patient was alert and oriented. She was hypertensive (200/90) and distressed by her headache. Her pupils were equal and reactive at $2 \mathrm{~mm}$ diameter. Ophthalmologic examination revealed a bitemperal hemianopsia but no ophthalmoplegia. The remainder of her examination was unremarkable. Skull radiographs demonstrated marked expansion of the sella tursica with erosion of the dorsum sella and clivus. A Computed Tomographic (CT) scan revealed a large, well defined, hyperdense mass in the sella and suprasellar region. There was minimal contrast enhancement and no subarachnoid hemorrhage, suggesting the diagnosis of hemorrhage into a pituitary adenoma (pituitary apoplexy).

Cerebral Magnetic Resonance Imaging (MRI) demonstrated a partially thrombosed giant aneurysm in the sella with suprasellar extension. This was confirmed by digital subtraction angiography. 
This case demonstrates the value of MRI as a non-invasive tool in differentiating vascular pathology from mass lesions in the central nervous system.

P91.

\section{Unilateral Creutzfeldt-Jakob Disease Presenting as Rapidly} Progressive Aphasia

\section{A. KIRK and L.C. ANG (Saskatoon, Saskatchewan)}

A 64-year-old man presented with a three day history of progressive Broca's aphasia, followed within 3 weeks by exclusively right-sided myoclonus, rigidity, and dystonia. By 4 weeks he was globally aphasic. He died within 7 weeks of onset. In the final week of his illness, rigidity and myoclonus became bilateral. CT and MRI of head were normal. SPECT showed diminished perfusion of the left hemisphere. EEG showed periodic discharges on the left. At autopsy, there was marked cortical spongiform change, neuronal loss, and gliosis throughout the left hemisphere and in the right occipital cortex. Elsewhere in the right hemisphere, spongiform change was non-existent to minimal. There was moderate spongiform change in the molecular layer of the cerebellar cortex, much more marked on the left. Amyloid and senile plaques were noted throughout the cerebral cortex. Clinical and pathological unilateral cerebral predominance extended to the ipsilateral cerebellum. CJD is an important consideration in differential diagnosis of rapidly progressive focal cortical signs associated with a movement disorder.

P92.

\section{Temporal Processing Impairment in a Case of Pure Word Deafness}

\section{PINARD, H. CHERTKOW, S. BLACK and V. WHITE-} HEAD (Montreal, Quebec; Toronto, Ontario)

Modern neurolinguistics research suggests that many of the "classic" aphasia syndromes could be better redescribed in terms of a breakdown of specific components of language processing. We have studied a 34-year-old woman with Pure Word Deafness (diagnosed by Dr. N. Geschwind) due to a static focal encephalitic lesion. While she conformed to the diagnosis on simple language testing, more in-depth neurolinguistic assessment revealed a different picture. She was found to have a basic impairment in auditory processing which prevented accurate recognition of phonemes as well as certain non-verbal sounds. Both deficits were related to a basic impairment in auditory processing; she could not distinguish sounds less than $128 \mathrm{~ms}$ apart (vs $3 \mathrm{~ms}$ apart for normal subjects). This deficit will be discussed in terms of theories of auditory perceptual processing.
P93.

Observations in Thirteen Patients with Parkinsonism and Basal Ganglia Calcification

\section{R.K. MOSEWICH, A.H. RAJPUT and J. RAVINDRAN (Saskatoon, Saskatchewan)}

The significance of basal ganglionic calcification (BGC) to extrapyramidal manifestations is uncertain. Most cases of $\mathrm{BGC}$ demonstrated on cranial computed tomography (CT) scans are idiopathic. However, many causes exist including hypoparathyroidism, congenital infections, birth anoxia, mitochondrial encephalopathy and carbon monoxide poisoning. Parkinsonian features are occasionally seen in association with BGC.

We identified from our movement disorders clinic 13 parkinsonian patients ( 5 male, 8 female) who had BGC on CT scan to discern if they differed from cases of idiopathic Parkinson's disease (IPD). The mean age of the patients was 72.2 years (range 66 - 78). On the basis of clinical finding, ten cases had IPD. Three cases had other clinical diagnoses which were striatonigral degeneration, olivopontocerebellar degeneration, and carbon monoxide poisoning. The average stage of parkinsonism was 3 and the average duration of the desease was 6.3 years. Depression was a common additional feature found in $6(46 \%)$ patients.

Of those cases diagnosed as IPD, all showed improvement with antiparkinsonian medications and none showed atypical features. It appears that BGC was an insignificant finding in these patients which did not change the overall course of their disease.

P94.

Trigeminal Sensory Neuropathy with a Pontine Lesion on MRI in a Patient with Probable Mixed Connective Tissue Disease

R.K. MOSEWICH, A. SHUAIB, W.P. OLSZYNSKI and I. MEISSNER (Saskatoon, Saskatchewan; Rochester, Minnesota)

A 35-year-old right handed lady presented with a one year history of insidious onset of right facial numbness associated with dysesthesias, Raynaud's phenomenon and recurrent oral ulcers. She had hypothyroidism and a 10 year history of discoid lupus erythematosus.

On physical examination she had subtle sclerodermatous features. Decreased pinprick sensation was present in the entire right trigeminal nerve distribution with light touch sensation less impaired. Muscles of mastication and corneal reflexes were spared. A subtle right facial droop was present. Mild proximal weakness in the upper limbs bilaterally was noted. Reflexes were brisk and plantar responses were flexor. Sensation and coordination testing was unremarkable.

Investigations performed revealed elevated serum ANA, SSA, SS-B and anti-RNP. Magnetic resonance imaging scan showed a hyperdense lesion in the right pons of $\mathrm{T} 2$ weighted imaging. 
The patient described has both clinical and serologic evidence of active connective tissue disease. Trigeminal sensory neuropathy has been well described as an early manifestation in patients with mixed connective tissue disease and progressive systemic sclerosis. It has been presumed to be due to a lesion in the trigeminal ganglion. Our case suggests the possibility that the symptoms may occur on the basis of central rather than a peripheral lesion.

\section{P95.}

Multiple Sclerosis in Descendents of a Mating Between "Ethnically" Resistant (Amerindian) and Susceptible (Scottish) Individuals

A.D. SADOVNICK, C. GREENBERG, C. BOURQUE and G.C. EBERS (Vancouver, British Columbia; Winnipeg, Manitoba; London, Ontario)

MS is due to genetic and environmental factors. Twin and family data suggest that MS susceptibility is probably oligogenic (influence of more than one locus).

The value of multicase MS families for linkage detection in complex traits remains uncertain. Such families may have several individuals homozygous at putative dominant or semi-dominant susceptibility loci, especially if the loci are common.

Matings between "ethnically" susceptible and resistant individuals, while rare, are informative. We present a family with 6 cases of MS over 2 generations. The mother was Scottish, the father was Cree. They had a total of 18 children, 5 of whom have MS. There are 49 grandchildren resulting from a total of 12 matings (7 with Amerindians; 5 with Caucasians of $\mathrm{N}$. European ancestry). One grandson has MS. The remaining grandchildren, all in their teens and 20's, are still in the "at risk" age period. While one cannot presuppose MS "susceptibility/ non-susceptibility" gene frequencies in either Amerindian or Scottish populations, such a mating is suggestive of a possible dominant effect.

The family will be presented with respect to the clinical aspects of MS and the informativeness for our understanding of the genetic susceptibility to MS.

P96.

Paroxysmal Kinesigenic Dystonia, "Paroxysmal Hyperglycinuria" and Multiple Sclerosis

\section{YEGAPPAN and T. CURRAN (St. John's, Newfoundland)}

Background: Paroxysmal dyskinesia as an initial manifestation of multiple sclerosis (MS) is reported but uncommon. We report a patient presenting with paroxysmal kinesigenic dystonia as an initial manifestation of MS who also had "paroxysmal" severe hyperglycinuria.

Results: A 41-year-old female presented with a three week history of recurrent involuntary spasms of her left hemibody occurring several times each day. The stereotypic spasm involved a sudden flexion at the elbow and wrist and hyperex- tension at the MCP joint with occasional spread to the leg. Events were precipitated by movement and lasted less than 1 minute. Interictal examination revealed a left internuclear ophthalmoplegia and cerebellar abnormalities. Evoked potentials, CSF analysis and MRI were all abnormal supporting the diagnosis of MS. Because of the unusual presentation and a sibling with "MS" a metabolic screen was preformed which revealed severe hyperglycinuria (HG). Three months later the patient was free of spasms and at this time urine, serum and CSF were normal for amino acids.

Conclusions: There is only one other report of paroxysmal dyskinesia associated with HG. We are unaware of any previous reported association between HG and MS. We will review the neurological manifestations of $\mathrm{HG}$ and the possible relationship it may have in our case.

P97.

Alterations in the Somatosensory Thalamus of Anaesthesia Dolorosa Patients

\section{Z.H.T. KISS, J.O. DOSTROVSKY, K.D. DAVIS and R.R. TASKER (Toronto, Ontario)}

Experimental studies in animals and humans have indicated that peripheral denervation results in reorganization of the somatosensory map at various levels of the CNS. It is thought that the representation of a body part adjacent to a region that is peripherally denervated expands into the deafferented area. To investigate this hypothesis, microelectrode recording and stimulating techniques were used to analyze the thalamic somatotopy in 100 patients undergoing stereotactic procedures for pain or movement disorder.

As a preliminary part of the study, the data from 9 patients with anaesthesia dolorosa (AD) were compared to 9 with Parkinson's disease (PD). There was no significant difference in tactile representation of face, mouth or hand between the 2 groups ( $p>.1$ ); however microstimulation induced paraesthesia in the face over a significantly $(\mathrm{p}<0.05)$ larger area of thalamus in the $\mathrm{AD}$ group $\left(\mathrm{V}^{2}: 9.6 \pm 8.4 ; \mathrm{V}^{3}: 4.7 \pm 3.8, \mathrm{~mm}\right)$ compared to the PD group $\left(\mathrm{V}^{2}: 2.4 \pm 2.4 ; \mathrm{V}^{3}: 0.9 \pm 1.5, \mathrm{~mm}\right)$. These results suggest that deafferentation results in alterations in thalamocortical processing of somatosensory information of the affected region. These changes may be involved in the pathophysiology of this type of deafferentation pain.

P98.

Diaphragmatic Evoked Potentials and Phrenic Nerve Conduction Studies in Patients with Sleep Apnea

F. GRAND'MAISON, V. SOLAND and J. REIHER (Sherbrooke, Quebec)

Central and obstructive sleep apnea (CSA, OSA) may result from any lesion within the respiratory loop from chemoreceptors to sensory nerves, brainstem nuclei, descending motor pathways, phrenic nerves and diaphragm. However, most patients 
with sleep apnea have no demonstrable neurological lesions on clinical or radiological examinations. Central and peripheral neural pathways to the diaphragm, which represent the efferent limb of the respiratory loop, have not been systematically assessed electrophysiologically in these patients.

Thirteen patients with CSA, 9 patients with OSA and 12 normals were studied. All patients but two siblings with an unclassified form of spinocerebellar degeneration and CSA has a normal neurological examination. Electrophysiological tests consisted of transcutaneous electrical stimulation of the phrenic nerves in the neck and of transcranial magnetic stimulation of the cerebral cortex to evoke diaphragmatic potentials.

Phrenic nerve conductions were normal in all patients. Diaphragmatic evoked potentials were abnormal only in the two siblings with spinocerebellar degeneration: no response in one sibling and only poorly developed DEPs in the other.

It is concluded that cortically evoked diaphragmatic potentials are normal in neurologically intact subjects with central and obstructive sleep apnea but may be abnormal in some patients with an underlying neurological lesion.

\section{P99.}

\section{The Spectrum of Adult Onset Niemann-Pick Disease}

O. SUCHOWERSKY, S. HASHIMOTO, B. CURRY and I. AUER (Vancouver, British Columbia; Calgary, Alberta)

Two families with clinically and/or autopsy proven adult onset Niemann-Pick disease (NPD) will be presented. In both families, a brother and sister were affected, with no other family history or consanguinity.

In family A, symptom onset occurred at age 40 in the female, and age 15 in the male. Both had progressive neurological dysfunction with ataxia, bradykinesia, chorea, sensorineural hearing loss, spasticity and dementia. Autopsy of both (at age 35 in the male, and 48 in the female) showed hepatosplenomegaly, and Niemann-Pick type C storage cells in the CNS, PNS, bone marrow, lymph nodes, spleen, liver.

In family $\mathrm{B}$, both sibs were noted to have asymptomatic hepatosplenomegaly and abnormal fundoscopic examination since early adulthood. Neurological symptoms, consisting of neuralgia, paraesthesia and tremor began in their mid forties. Peripheral neuropathy was confirmed clinically and on EMG testing. Bone marrow showed sea-blue histiocytes. Sphingomyelinase activity was absent.

The current concepts about adult onset NPD will be discussed.

\section{P100.}

Para-infectious Leukoencephalitis - A Case Study with MRI and Pathologic Findings

J. RAVINDRAN, A. SAXENA, N. LOWRY and L. ANG (Saskatoon, Saskatchewan)

Para-infectious leukoencephalitis consists of a spectrum of disorders with varying clinical symptoms, severity, sites of lesion and natural history. We report a six-year-old girl who presented with unsteadiness and tendency to fall to the left. Two days later she developed vertigo and medial deviation of left eye. Two weeks prior to this she had an upper respiratory infection. Examination revealed normal higher function, mild restriction of the left lateral rectus, mild left arm drift, normal reflexes, sensation and flexor plantar response. She had signs of cerebellar incoordination with broad based ataxic gait. CSF analysis showed lymphocytic pleocytosis. MRI T2 images showed a contrast enhancing focal lesion in the left middle cerebellar peduncle. Biopsy showed perivenous demyelination. Her symptoms improved well without treatment. MRI two months later showed some resolution of the lesion in the left middle cerebellar peduncle and a new lesion in the right cerebellum.

This case is one of the few cases with pathologic confirmation. The MRI findings did not correlate with clinical symptomatology contrary to previous reports.

P101.

\section{Limbic Encephalitis: MRI as a Diagnostic Aid}

\section{J.A. ESPINOSA, A. SALAZAR and R. DEL CARPIO (Montreal, Quebec)}

The authors report their experience with two cases where the clinical diagnosis of paraneoplastic limbic encephalitis was supported by consistent findings on magnetic resonance (MRI). Both patients had progressive dementia and memory loss that preceded the diagnosis of a primary neoplastic lesion outside the brain. CSF samples showed few lymphocytes and slightly increased protein in both patients. Viral studies were negative. MRI disclosed hyperintense signals on the mesial temporal lobes on T2 and proton density modalities. Lack of edema, mass effect and parenchymal hemorrhage made the diagnosis of herpes encephalitis and brain neoplasia highly unlikely. Findings on CT scan were not constant. We believe that in these two cases, the clinical presentation and MRI correlations were strong enough to preclude a surgical intervention for tissue diagnosis.

P102.

\section{Cyclosporin A Treatment of CNS Sarcoidosis}

\author{
R.Z. KERN, W. MONTANERA, V. BYKERK and D. KIM \\ (Mississauga; Toronto, Ontario)
}

Neurological manifestations occur in 5\% - 6\% of patients with sarcoidosis. This includes central nervous system involvement, basal meningitis, multiple cranial neuropathies, peripheral neuropathy and myopathy. Corticosteroids are the mainstay of treatment, although various immunosuppressive agents and/or radiotherapy have been used with varying success. There are several reports of CNS sarcoidosis treated with Cyclosporin A.

We report a young male patient who presented with multiple cranial neuropathies due to sarcoid basal meningitis. 
Cyclosporin A produced clinical improvement and resolution of basal meningitis as visualized by gadolinium-enhanced cranial MRI.

P103.

Rapidly Progressive CNS Complications in Idiopathic Hypereosinophilic Syndrome

\section{H. AL-RAHI and M. VEILLEUX (Montreal, Quebec)}

We report a 54-year-old man with idiopathic hypereosinophilic syndrome (HES) who died following numerous embolic strokes less than 5 weeks after initial diagnosis. He was admitted to the hospital because of low grade fever, myalgias, arthralgias, loss of appetite, and a 2-day history of numbness in the right shoulder and right hand clumsiness. On admission, he was confused and his memory impaired. On examination, he had a moderate weakness of the right shoulder muscles, brisk reflexes in the right arm, conjunctival hemorrhages, and splinter hemorrhages in the tips of the fingers and toes. Complete blood count revealed 96,000 eosinophils. Blood and stool cultures, serial echocardiograms, abdominal ultrasounds, muscle biopsy and bone marrow aspiration were normal, and $\mathrm{CT}$ of the head showed a right frontal hypodensity. Despite being started on steroids and hydroxyurea, he developed 5 days later a rightsided hemiplegia and on the next day, he became comatose. A repeat CT of the head revealed bilateral watershed infarcts and a few small hemorrhages. He died 3 weeks later without regaining consciousness. Unlike previously reported patients with HES, our patient developed rapidly progressive, severe CNS complications despite aggressive treatment.

P104.

Central Pontine Myelinolysis: Delayed Onset After Correction of Hyponatremia

H. AL-RAHI, C.H. CHALK, P. BOURGOUIN, J. RICHARDSON and M. VASILEVSKY (Montreal, Quebec)

Central pontine myelinolysis (CPM) typically develops during or within a day of rapid correction of the hyponatremia. We report 2 new observations: (1) there may be a substantial delay between correction of hyponatremia and the onset of CPM; (2) the MRI lesions may enhance with gadolinium.

A 31-year-old alcoholic woman developed confusion and seizures. Her $\mathrm{Na}^{+}$was $105 \mathrm{mEq} / \mathrm{L}$ and it was raised to 138 $\mathrm{mEq} / \mathrm{L}$ in 12 hours with 3\% saline. Mental status improved promptly and her seizures stopped. She was discharged after 5 days, behaving normally. Six days later, she became progressively unresponsive over 12 hours. She was drowsy and mute, would open her eyes to voice, but did not follow commands. When stimulated she moved her limbs vigorously and required restraints. Both plantar responses were strongly extensor. Serum $\mathrm{Na}^{+}$was $135 \mathrm{mEq} / \mathrm{L}$, and other blood chemistries, toxic screen, CSF, and cranial CT were normal. An EEG showed generalized slowing, without epileptiform features. MRI of the brain revealed gadolinium-enhancing lesions in the pons, midbrain, thalami, and parietal white matter. During the next week, she developed spastic quadriplegia. Thereafter her neurological condition did not change, and seven weeks later she died of pneumonia. At autopsy, typical lesions of CPM were found in the upper brainstem and thalami.

\section{P105.}

\section{A Rare Cause of Hyponatremic Obtundation - A Case Report and Literature Review}

D.F. LOUW, V.E. SANGALANG and R.A. PURDY (Halifax, Nova Scotia)

We present a case of "spontaneous" bilateral adrenal hemorrhage manifesting with acute neurological symptoms.

A 61-year-old woman underwent a total knee replacement for osteoarthritis. On the 5th post-operative day it was noted that she had a swollen, tender left calf. Deep venous thrombosis was confirmed on venography and intravenous heparin was started. On the ninth post-operative day she developed abdominal pain and confusion, which progressed over two days to obtundation.

CT of the brain was normal, and the serum sodium was 116 $\mathrm{mmol} / \mathrm{L}$. She continued to deteriorate and developed hypoxia, atrial fibrillation and shock which was refractory to therapy. The causes of hyponatremia were sought and an exploratory (negative) laparotomy was undertaken. The patient died on the 16th post-operative day, and an autopsy disclosed bilateral adrenal hemorrhage.

Further clinical and pathological data will be presented along with a review of the pertinent literature. Heparin associated adrenal hemorrhage is rare, but deserves further attention because it can present with neurological symptoms.

P106.

Isolation of Peroxidase-Positive Astrocyte Granules from Intact Rat Brain and Cysteamine-Treated Neuroglial Cultures

\section{S. CISSÉ and H.M. SCHIPPER (Montreal, Quebec)}

A subpopulation of astrocytes in the aging periventricular brain and in cysteamine (CSH)-treated glial cultures contain autofluorescent cytoplamic granules that exhibit an affinity for chrome alum hematoxylin ( $\mathrm{CAH})$ and non-enzymatic peroxidase activity. The intense endogenous peroxidase activity in these glial inclusions is capable of oxidizing neutral catechols to neurotoxic semiquinone radicals, implicating the peroxidasepositive astrocytes in the pathogenesis of free radical-related neurodegenerations. Although shown to be histochemically destinct from lipofuscin, the lack of pure preparations of these glial inclusions has hindered the elucidation of their precise chemical constituents. Using sucrose gradient fractionation and density centrifugation on percoll, we obtained enriched preparations of astrocyte cytoplasmic granules from intact rat brain and CSHtreated astrocyte cultures. The presence and relative purity of 
these inclusions were confirmed by lasar scanning confocal microscopy for red autofluorescent granules, diaminobenzidine histochemistry for non-enzymatic peroxidase activity, and $\mathrm{CAH}$ staining. As in situ, the purified material was argyrophilic and did not stain for lipids or glycoproteins. Isolation of these astrocytic inclusions should permit a more thorough characterization of their biochemical contents.

\section{P107.}

\section{Survival and Differentiation Effects of CNTF on a Human Neural Cell Line}

\section{G. LAWRANCE, R.J. RIOPELLE, S. GUPTA and P.M. RICHARDSON (Kingston, Ontario)}

Ciliary neurotrophic factor (CNTF) is a member of a family of neuropoietic cytokines that includes leukemia inhibitory factor (LIF), interleukin-6, and oncostatin. Where a human neural cell line was used as a target in serum-free conditions in vitro, CNTF was not required for survival for periods up to 14 days, but during that time induced neurite formation and cholinergic differentiation. CNTF bound to the cells with two affinities displacement of [ ${ }^{125} \mathrm{I}$ )-CNTF by unlabelled CNTF occurred in the range of $100 \mathrm{pM}$, and in the range of $2 \mathrm{nM}$. When the cells were maintined in serum-free conditions for periods greater than 14 days, cell death occurred that could not be reversed by CNTF or Nerve Growth Factor (NGF) alone, but was inhibited in the presence of both CNTF and NGF. NGF had no influence on neurite growth by the cells except in the presence of CNTF where the influence was greater than in the presence of CNTF alone.

Survival and differentiation effects of CNTF on a human neural cell line can be distinguished temporally. CNTF causes differentiation which includes priming the cells to respond to NGF. The priming response is essential for reversing delayed cell death in limiting conditions. NGF has both survival and differentiation effects on the cells in the presence of CNTF.

Supported by: the Canadian Federal Networks of Centres of Excellence Program in Neural Regeneration and Functional Recovery.

\section{P108.}

Pluripotential Precursor Cells as a Source of GeneticallyAltered Neuronal Tissue For CNS Grafting

D.J. MORASSUTTI, D.S.K. MAGNUSON, W.A. STAINES, K.C. MARSHALL and M.W. MCBURNEY (Winnipeg, Manitoba; Ottawa, Ontario)

One potential therapeutic approach for human neurodegenerative disorders is replacement of lost neuronal populations by appropriate tissue grafts. Preliminary results using human fetal tissue grafts for Parkinson's disease have been encouraging, but this avenue is hampered by ethical considerations and donor tissue availability. Consequently, attempts have been made to find alternate donor material. We have investigated the use of embry- onal carcinoma (EC) cells as a source of tissue which can be used to deliver exogenous gene products to the mammalian CNS. EC cells are immortal, grow in culture and can be efficiently induced, by retinoic acid (RA), to irreversibly differentiate into non-tumorigenic cells including post-mitotic neurons. These EC-derived neurons contain a spectrum of neurotransmitters closely resembling those found in the mammalian forebrain. We demonstrate that neural cells derived from cultured EC cells survive following grafting into the adult rat striatum, no tumours develop, and the differentiated cells continue, in vivo, to express foreign genes transfected into their parental EC cells. The neurons in these grafts express a variety of neurotransmitters and mature to acquire fully developed neuronal electrophysiological properties. Our results suggest genetically altered neuronal precursor cell populations may be useful as an alternative source of CNS grafting donor tissue.

Supported by: NCI, MRC (Canada), the Canadian Centre of Excellence for Neural Regeneration and Functional Recovery.

P109.

\section{Fascicular Carpal Tunnel Syndrome}

F. GRAND'MAISON, M.L. LEBEL and K. PHAN (Sherbrooke, Quebec)

Clinical findings in patients with carpal tunnel syndrome (CTS) may be limited to, or may predominate in, one or two fingers. This observation suggests selective involvement of some fascicules in the median nerve at the wrist. We compared median nerve conduction studies in the most clinically affected digit(s) with those of less affected digits in 27 such patients.

Digital nerve action potentials (DNAPs) from each digit $\left(I_{R}\right.$, $\mathrm{I}_{\mathrm{M}}, \mathrm{II}_{\mathrm{M}}, \mathrm{III}_{\mathrm{M}}, \mathrm{IV}_{\mathrm{M}}, \mathrm{IV}_{\mathrm{U}}, \mathrm{V}_{\mathrm{U}}$ ) were evoked by stimulating the radial $(\mathrm{R})$, median $(\mathrm{M})$ or ulnar $(\mathrm{U})$ nerves at the wrist at a distance of $130 \mathrm{~mm}$.

In 2 patients with symptoms and signs limited to one digit, no DNAP could be elicited in the involved digit while median nerve conduction studies to the other 3 digits were minimally (one patient) or moderately (one patient) altered. In the remaining 25 patients, upon median nerve stimulation, DNAP latencyto-peak was significantly longer in the most clinically affected digit(s) than in the less affected digits (difference $=.27 \mathrm{~ms}, \mathrm{p}<$ .003 ).

It is concluded that some fascicules of the median nerve are preferentially involved in many patients with CTS (fascicular CTS). The diagnostic yield may therefore be increased by recording from the most clinically affected digit(s).

P110.

\section{A Quantitative EEG Comparison Between Alpha Pattern Coma and the Normal Awake State}

\section{M.J. MCKEOWN and G.B. YOUNG (London, Ontario)}

Alpha pattern coma (APC) is an uncommon clinical-EEG finding in comatose patients which superficially resembles the 
recordings of normal, awake individuals. In contrast to normals, the alpha $(8-13 \mathrm{~Hz}$ waves) in APC shows a more widespread topography and no reactivity to stimuli. To determine other significant differences between the two groups, we employed computerized analytical techniques.

We studied 5 normal individuals and 5 patients with APC who died without recovering consciousness. Using the REEGAL (McKeown and Young, 1993) tool, we quantified paper traces of the two groups for computer analysis. Left and right parietal-occipital derivations were used to compute coherence, phase and power spectral information for each person. Front-toback comparisons were also obtained for one individual from each group.

The APC group significantly differed from normals in the following respects: (a) broader frequency distribution within the alpha range, (b) more prominent delta and (c) lower left-right peak coherence in the alpha range.

Quantitative analysis revealed fundamental differences between normal alpha and the alpha in APC which were not readily appreciated on the raw trace. Theoretical implications will be discussed.

P111.

Type III Intermittency: A Nonlinear Dynamic Model of EEG Burst Suppression

A.D. RAE-GRANT and Y.W. KIM (Allentown; Bethlehem, U.S.A.)

We studied EEG burst suppression patterns from 9 comatose patients using nonlinear dynamic techniques. These EEG records showed many phenomena characteristic on nonlinear systems, including sensitivity to initial conditions, similarity across scale, self-organization, and intermittency. Burst and "burst to burst" durations showed an asymmetric distribution with a decreasing tail of increasing duration. We attempted to interpret the histograms from the standpoint of intermittency classifications of iterated dynamical maps. The absence of any conspicuous maximal cut-off duration suggested a type III intermittency. We determined the power-law exponent of the decreasing tail to be near to the predicted exponent of -1.5 for type III intermittency (-1.5 to -3.0$)$. We evaluated long durations of EEG in a patient in a pentobarbital coma and found that loglog plots of burst durations closely followed a type III pattern (i.e. showed an exponent of -1.5). In addition, we developed a nonlinear algorithm which robustly models a burst suppression pattern based on a low dimensional return map.

Burst suppression pattern appears to be the constrained activity of a nonlinear system at the transition to chaos, one showing a specific intermittency pattern. This work may help in developing a framework for elucidating the origin of other EEG patterns.
P112.

Stimulation-Evoked Changes in Intrinsic Optical Signals in the Human Cerebral Cortex

\author{
F.E. LeBLANC, B. MACVICAR, T. WATSON, O. WILLIAMS \\ and B. SPENCE (Calgary, Alberta)
}

Neuronal activity in several systems has been shown to be associated with changes in the optical properties of nervous tissue. We have asked whether activity in the human neocortex is also associated with changes in intrinsic optical signals. The cortex of patients was exposed during operations to remove seizure foci for the treatment of intractable epilepsy. Punctate bipolar stimulation is normally performed during these operations to map key motor and sensory areas. During electrical stimulation, video imaging of the cortex was performed to ascertain whether there were changes in the reflectance of the tissue during stimulus-evoked activity. Digitized images were averaged during control periods. During stimulation there was a progressive decrease in tissue reflectance around the stimulating electrodes. This change was repeatable and was graded with changes in stimulus intensity. These results indicate that noninvasive video imaging of changes in intrinsic optical signals reflect physiological events related to cortical neuronal discharge. The application of this technique to functional mapping of the human cerebral cortex at surgery will be demonstrated.

\section{P113.}

Suppressive Effect of the MEP on the SSEF Interferes with Selective Monitoring of the Spinal Cord in Rats

\section{KOYANAGI and C.H. TATOR (Toronto, Ontario)}

The purpose of this study was to standardize the method of spinal cord monitoring with sensory evoked potentials in the rat. Twenty-one Wistar rats were anesthetized with $\alpha$-chloralose and urethane. The rats were paralysed with pancuronium bromide and mechanically ventilated. Sensory evoked potentials following sciatic nerve stimulation were recorded epidurally from $\mathrm{T} 9$, $\mathrm{C} 2$, cerebellum (CEP) and sensorimotor cortex (SSEP). In 7 rats, multiple recordings of CEP and SSEP over the frontoparietal and cerebellar cortices were performed to determine the optimal recording sites. The first two negative peaks of spinal evoked potential at $\mathrm{C} 2$ were markedly attenuated by sectioning both the dorsal and lateral columns at C7-T1. The CEP reflected mainly conduction in the ventral cord. The large negative peak of the SSEP at $18 \mathrm{msec}$ was markedly attenuated by dorsal column section. In 10 rats, the motor evoked potential (MEP) was also recorded from T9 epidural space. MEP performed immediately before caused suppression of the cortical response of the SSEP. This study shows that the ventral and dorsal integrity of the rat spinal cord can be monitored with multilevel recordings of sensory evoked potentials. MEP should not be used for concurrent monitoring when the MEP is produced by stimulating the same sensorimotor cortex from which the SSEP is being recorded. 
P114.

Activation of Ipsilateral and Contralateral Forelimb Muscles by Transcranial Magnetic Stimulation

\section{S.S. HAGHIGHI, N. MALUGANI, S. ROTH and J.J. ORO (Missouri, Colombia)}

We studied the effects of transcranial magnetic stimulation of the motor cortex on ipsilateral and contralateral forelimb extensor muscles in nine lightly anesthetized cats. Magnetic stimulator operated at $100 \%$ intensity was used through an eight-shaped coil, which was placed tangentially over the scalp on the right side. Evoked muscle responses were recorded bilaterally from forearm extensors. Bilateral activation of extensor muscles were readily obtained in all animals under light level of anesthesia. The onset latencies were $7.3 \pm 1.1$ and $7.07 \pm 0.8 \mathrm{msec}$ for the contralateral and ipsilateral muscles, respectively. These values were not statistically different $(P>0.05)$. The muscle amplitudes were unstable in magnitude, nevertheless, they did not show any significant difference between the two sides $(P>$ $0.05)$. The ipsilateral muscle response with non-significant latency difference to the contralateral side suggests bilateral simultaneous activation of descending motor pathways that serve the forelimb muscles on both sides. Furthermore, this process is mediated through synaptic relays, since they demonstrated to be extremely sensitive to volatile anesthetics.

\section{II - 3 NEUROSURGERY}

\section{P115.}

\section{Periodontoid Synovial Cyst (Ganglion)}

A.M. KAUFMANN, W.C. HALLIDAY, M. WEST and D. FEWER (Winnipeg, Manitoba)

Periodontoid synovial cyst (ganglion) are rare; we present as case involving an otherwise healthy 52-year-old male who had a progressive and fluctuating course of neurological deterioration associated with episodic neck pain. This culminated in severed limb ataxia, weakness, hand muscle wasting and lower cranial nerve palsies. There was no history of trauma. Investigations included an LP which resulted in a brief respiratory arrest. CT myelography failed to assess the upper cervical area and was thus non-diagnostic. Magnetic resonance imaging demonstrated a cystic periodontoid lesion associated with thickening of the quadrate ligament. Initial management included aspiration of the cyst which provided only modest and temporary improvement. Definitive decompression achieved by an anterior transoral approach had a very good clinical result. Histopathology demonstrated a synovial cyst within the ligament and adjacent fibrovascular tissue. In summary, periodontoid synovial cysts (ganglion) should be included in the differential diagnosis of extra-medullary, extra-dural mass lesions in the region of the foramen magnum and upper cervical cord. Decompression by a direct anterior transoral approach is advocated.
P116.

\section{Functional Outcome in Patients with Congenital Hydrocephalus}

\section{F. GENTILI, S. LIAO and B. JHAWAR (Toronto, Ontario)}

Since the introduction of valve regulated shunts in the $1950 \mathrm{~s}$ the management of patients with congenital hydrocephalus has improved significantly. While the result of treatment of these patients from infancy to adolescence has been well studied there is less information on the long-term functional outcome of these patients throughout adulthood. Eighty patients with congenital hydrocephalus have been studied prospectively since their referral to an adult neurosurgical institution following their 18th birthday. There were 33 females and 47 males followed up to a mean age of 25 years (range $18-39$ years). Of this group of patients 32 had not had a shunt revision after age 18 . The average shunt revision-free period was 11.6 years (range $5-25$ years). The average number of shunt revisions after age 18 in the remaining patients was 2.39 revisions per patient. Indications for revision were distal (34\%) and proximal $(23.2 \%)$ obstruction, disconnection/migration $(9.9 \%)$, infection $(7.7 \%$ ) and numerous less frequent causes $(26.6 \%)$.

Fifteen percent of patients had formal neuropsychological testing. The average self reported functional independent measure (FIM) score was 100.2 (range 58 - 126). Three patients had uneventful pregnancy with delivery of normal infants. Fifty percent of patients were attending or had completed high school and $20 \%$ had entered college. The majority of patients were living independently with their families or in group homes with only $10 \%$ institutionalized. In summary, the long-term functional outcome of patients with congenital hydrocephalus is good with many patients being capable of leading normal independent and productive lives.

P117.

Reconstruction of Cranial Bone Defects in Children Using Fixed Autogenous Bone Grafts

J.T. RUTKA, J. POSNICK, J. GOLDSTEIN and D. ARMSTRONG (Toronto, Ontario; Washington, D.C., U.S.A.)

To determine the relative efficacy of autogenous fixed cranial bone grafts in children, we reviewed our experience with 27 consecutive patients between 1986 and 1990. The causes of the skull defects included: encephalocele ( 6 patients), trauma (7 patients), tumor ( 8 patients), fibrous dysplasia ( 2 patients), postsynostectomy defects ( 2 patients), osteomyelitis (1 patient), and Reye's syndrome with bone flap resorption (1 patient). Skull defects were reconstructed in all patients using fixed autogenous cranial bone grafts. In the majority of patients, the bone grafts were harvested as full-thickness calvarial grafts which were split and used for reconstruction of both donor and recipient sites. In the initial 5 patients, the bone grafts were fixed with interosseous wires, and in the remainder with a combination of 
titanium mini- and micro-plates and screws. All patients underwent clinical and CT scan documentation of their skull defects before and immediately after surgery, and at least 1 year later. The average preoperative skull defect measured $33 \mathrm{~cm}^{2}$ (range $2.5-114 \mathrm{~cm}^{2}$ ). Follow-up ranged from 12 to 66 months (mean 31.4 months). Complications were minimal. In 24 of 27 patients, clinical examination and CT scans showed no evidence of skull defect or appreciable irregularity of donor or recipient sites. Two patients had documented small regions of graft resorption. One patient had palpable skull contour irregularities but without a bony defect. We conclude that the repair of skull defects in children using fixed autogenous cranial grafts is a reliable method of cranial vault reconstruction which is associated with minimal morbidity. Although we prefer mini- and micro-plates and screws for fixation, the grafts fixed with interosseous wires did equally well.

\section{P118.}

The Management of Acute Abdomen in the Paediatric Patient with Ventricular Peritoneal Shunt

\section{F.B. MAROUN, H. LAISHRAM, R. KENNEDY, D. PRICE and J.C. JACOB (St. John's, Newfoundland)}

Many children with VP shunt develop acute abdominal pain mimicking surgical conditions forcing the surgeon into a diagnostic quandary. In a retrospective analysis of $250 \mathrm{VP}$ shunted patients between 1980 and $1992-17$ were due to acute abdomen and 5 for acute or chronic pain. Further study of these 17 patients showed 7 cases of pseudocyst or CSF - 4 diagnosed by ultrasound, 3 by laparotomies, 6 for peritonitis, 2 cases of intra-abdominal abscesses and 2 for intestinal obstruction. With the aid of ultrasound examination and initial diagnosis unnecessary laparotomies were avoided in 7 instances. Studies shows that all VP shunted patients who presented with acute abdomen should be presumed to have distal shunt infection until proven otherwise and frequent use of ultrasound in assessment will enable a correct diagnosis thus reducing the need for laparotomy.

\section{P119.}

\section{Communicating Syringomyelia: Resolution after Shunt Revision}

\section{J.A. ESPINOSA, J.P. FARMER and J.L. MONTES (Montreal, Quebec)}

Three patients with myelomeningocele, shunted hydrocephalus and Chiari II malformation presented with isolated progressive spinal symptoms. None exhibited symptoms suggestive of shunt malfunction.

All were found to have non-functioning ventriculoperitoneal shunts and asymptomatic compensated hydrocephalus as the source of a syrinx. After shunt revision, the cavity collapsed in two patients and in the othr persisted partly. All patients improved symptomatically, hours after shunt revision.
Patients with myelomeningocele and Chiari II malformation may not show classical signs or symptoms of hydrocephalus at the time of shunt malfunction if the ventricular pressure is dissipated into a communicating syrinx. In dealing with hydromyelia in patients with myelodysplasia and Chiari malformation, verification of shunt patency is extremely important before considering other surgical alternatives.

P120.

Experience with the Use of Lumboperitoneal Shunt as Primary Treatment for Cranial Cerebrospinal Fluid Leaks

K.K.V. REDDY, M. WEST and R. DeVILLIERS (Winnipeg, Manitoba)

Cranial cerebrospinal fluid (CSF) leaks have been treated with a variety of surgical procedures when conservative management fails. Lumboperitoneal shunts (LPS) have also been utilized but indications for this procedure are unclear from the literature. The authors undertook a retrospective review of their experience with the use of LPS as the primary and definitive treatment of cranial CSF leaks. There were seven patients with a mean age of 55 years. Five of the patients were female and two were male. Three of the patients had post-operative CSF leaks. In three others the leak was spontaneous, and in one patient it was post-traumatic. The portal of egress of CSF was the nose in all but one patient (in whom the CSF leaked into the subgaleal space). All the patients except one had excellent results with a mean follow-up of 26 months. One patient developed severe pneumocephalus necessitating eventual removal of the shunt system. The authors feel tha LPS is a simple procedure associated with minimal morbidity though pneumocephalus and meningitis are potentially serious complications that occur rarely. The authors feel that LPS can be used as primary and definitive therapy for cranial CSF leaks with acceptable morbidity.

P121.

A Case of Acute Subdural Hematoma with Idiopathic Thrombocytopenic Purpura: A Dangerous Combination

\section{E.W. PETERSON, S.A. GEORGANOS and J. BORMANIS (Nepean; Ottawa, Ontario)}

Acute subdural hematoma (ASDH) is a neurosurgical emergency. Successful treatment of ASDH complicated by Idiopathic Thrombocytopenic Purpura (ITP) has not been reported in the literature. This unique case involved an active 68-year-old man who had a minor head injury. He became rapidly obtunded with left side hemiplegia and purpura. He had been treated in the past for chronic ITP. A CT scan revealed a large right sided ASDH with displacement of the midline structures to the left without brain stem compression. His platelet count was $9 \times 10^{9} / \mathrm{L}$. It was elected to delay surgical evacuation until the platelet level could be elevated to one that would preclude intraoperative complications due to bleeding. He was treated with corticosteroid, I.V. 
gamma globulin followed by platelet transfusion. After a 12 hour period a platelet level of $70 \times 10^{9} / \mathrm{L}$ was reached. At operation no difficulty was encountered. The patient made a good recovery without neurological sequelae. A subsequent CT scan revealed no recurrence of the subdural. The patient had a splenectomy 10 days later and he has been in satisfactory condition since.

\section{P122.}

Neurologic Deterioration in Severe Head Injury Despite Adequate Global Cerebral Blood Flow: Preliminary Findings with Quantitative Somatosensory Evoked Potentials

\section{S. KONASIEWICZ, R. MOULTON and A. BAKER (Toronto, Ontario)}

All patients with a severe closed head injury (GCS $\leq 8$ ) admitted to our neurosurgical intensive care unit are monitored with a standard protocal including continuous monitoring of somatosensory evoked potentials (SSEP) and twice daily determinations cerebral blood flow (CBF) using the nitrous oxide clearance method. Median nerve SSEPs are quantitatively assessed by summing peak to peak amplitudes.

Nine patients who died or were severely disabled were monitored from 1 - 12 days. SSEP amplitude deteriorated $63.2 \% \pm$ $26.5 \%$ from admission to the end of monitoring. In 4 of 9 patients, despite hyperemic levels of CBF throughout the monitoring period, reductions in SSEP activity of $47 \%, 76 \%, 80 \%$, and $87 \%$ took place. In 2 patients, the greatest reduction in SSEP activity occurred prior to a period of reduced blood flow. In 2 other patients, ischemia was followed by a period of hyperemia during which most SSEP activity was lost. One patient with a CBF of $14 \mathrm{cc} / 100 \mathrm{~g} / \mathrm{min}$., had markedly reduced SSEPs at the time of admission, and soon expired.

These results may indicate that an early ischemic period (detected in only 2 patients) precedes a reperfusion hyperemic state during which SSEP deterioration occurs. Alternately, in a proportion of severely head-injured patients, pathophysiological processes other than ischemia/reperfusion may cause neurologic deterioration.

\section{P123.}

Comparison of a Quantitative Measure of Somatosensory Evoked Potentials and Glasgow Outcome Scores in Severe Head Injury

\section{S. KONASIEWICZ, R. MOULTON and P. SHEDDEN (Toronto, Ontario; Houston, U.S.A.)}

From 1987 to 1992, patients with a severe closed head injury (GCS $\leq 8$ ) admitted to our neurosurgical unit were monitored with continuous median nerve somatosensory evoked potentials (SSEPs). A computer program was used to quantitatively assess SSEPs by summing peak to peak amplitude for each hemisphere. Glasgow Outcome Scores (GOS) at three months were compared to SSEP activity using ANOVA.

Of a total of 72 patients, 17 had good or moderate outcome, 22 were severely disabled, and 33 were vegetative or dead. Initial SSEP responses were not significantly different between outcome groups. SSEP responses tended to decrease over time in a gradual fashion, such that final SSEP responses measured at the end of the monitoring period (mean 6.2 days) were significantly different between outcome groups $(p=0.001$, both hemispheres, $p=0.004$, best hemisphere, $p=0.001$, worst hemisphere).

The results indicate that automated quantitative measurement of SSEPs correlates with outcome. Furthermore, delayed rather than early post-injury SSEP measurements are correlated with outcome, suggesting that the observed decline in SSEP activity during the period of monitoring is a major determinant of outcome in a significant number of patients.

P124.

Epidural Hematoma (EDH) in Children and Adolescents: Assessment of Outcome Determinants

\section{A.L. AMACHER (Danville, U.S.A.)}

A rural Level I Trauma Unit, covering an area of $13,500 \mathrm{~km}^{2}$, provides opportunity to examine factors presumed to affect outcome in those whose primary intracranial pathology is epidural hematoma. For varied reasons, despite frequent direct scene-tohospital helicopter transfer, injury-to-operation intervals exceeded 2 hours in all 24 consecutive cases $(7 \mathrm{~m}-19 \mathrm{y})$, exceeded 3 hours in 22, 4 hours in 13,6 hours in 10, and 12 hours in 7. Clot volumes exceeded $30 \mathrm{ml}$ in 21 , and $75 \mathrm{ml}$ in $8.23 / 24$ hematomas were of arterial origin. 21 of 24 patients are normal. There is no linear correlation between outcome and treatment interval, or location and size of clot, for 16 of 17 patients operated upon less than 12 hours from injury; one adolescent with post-traumatic cognitive deficit had significant diffuse axonal injury. Mortality was $1 / 24(4.2 \%)$, where referral delay was more than 36 hours. Of patients operated upon $12+$ hours post injury, 4 are normal, 2 have residual, improving cognitive difficulties, one is dead.

A concurrent group of 10 patients with small EDH $(<30 \mathrm{ml})$ not operated, are normal. Implications will be discussed.

P125.

Cortical Mapping under Local Anesthesia for Resection of Lesions in Eloquent Cortex

A.M. LOZANO, M. BERNSTEIN, C. BENEDICT and M.C. WALLACE (Toronto, Ontario)

We investigated the role of intra-operative cortical mapping in 24 patients undergoing surgery for lesions in eloquent cerebral cortex from 1991 to 1992 . There were 16 males and 8 females with ages from $25-72$. The lesions were in the frontal 
lobe in 13 patients, parietal lobe in 8 cases and 3 in the temporal lobe. Twelve patients presented with a seizure, 5 with motor deficits, 5 with speech deficits, one with an intracerebral hemorrhage and one with impairment of higher cognitive functions. The diagnoses were glial neoplasm in 10 patients, metastatic tumour in 9, vascular malformation in 3, meningioma in 1 and glial tumour and radionecrosis in 1. Patients underwent cortical mapping using monopolar stimulation with currents from $1-12$ milliamps, pulse width of $200 \mathrm{usec}$ and frequencies of $50-100$ hertz. Cortical stimulation permitted the identification of motor cortex, sensory cortex and both frontal and temporal speech areas. This information together with the ongoing physiological monitoring was important for surgical decision making. We conclude that awake craniotomy with brain mapping allows intraoperative localization of brain function and permits surgical approach to lesions in eloquent areas of the cortex. This technique may allow more aggressive approach to lesions that are otherwise considered unexcisable and may be associated with less morbidity than than observed with general anesthesia.

\section{P126.}

\section{Pseudo-CSF Rhinorrhea: A Previously Unrecognized Complication of Cranial Base Surgery}

\author{
M.D. CUSIMANO and L. SEKHAR (Toronto, Ontario; \\ Pittsburgh, U.S.A.)
}

Cranial base surgeons recognize the potentially fatal consequences of cerebrospinal fluid (CSF) leakage and have developed a variety of methods to prevent it.

We have identified 11 patients who complained of rhinorrhea associated with exertion following surgery for lesions of the cranial base. All patients had undergone exposure, mobilization, or resection of their petrous or cavernous internal carotid artery and division of the greater superficial petrosal nerve. Several patients underwent invasive investigations and had "unsuccessful" surgery for presumed CSF leakage.

This paper provides: (1) a clinicopathological correlation which suggests that the symptoms reflect a nasal hypersecretion syndrome caused by a relative disturbance of cranial autonomic pathways; and (2) a detailed description of the clinical syndrome so that clinicians might avoid unnecessary investigations and surgery.

\section{II - 4. SPINAL CORD}

\section{P127.}

\section{Lumbar Discogenic Degenerative Cysts}

\section{K.K.V. REDDY and R.B. DUNLOP (Hamilton, Ontario)}

The authors describe two cases of cystic lesions in the lumbar region arising from the disc space. Both patients were in their late twenties at the time of presentation. The presenting features were those of radicular pain and weakness in the distribution of the first sacral nerve root (S1) in both cases. Clinical examination revealed a decrease in the power in the muscles supplied by the first sacral nerve root along with decreased ankle jerks. Radiologic studies revealed what appeared to be sequestrated disc fragments. At surgery, in both patients, there were large masses in the axilla of the nerve root of S1. The mass appeared like a sequestered disc fragment but when incised, thick straw coloured fluid extruded. In both cases there was communication with the disc space. There was no connection to the facet joint synovium. Histopathology revealed disc material with the cystic space lining showing hemosiderin granules suggesting previous hemorrhage. Both patients have no further radicular features and have returned to their previous occupations. To the authors' knowledge, such lesions have not been described de novo previously though one such case has been reported post-operatively previously. We speculate that a large disc protrusion with a hemorrhage in it could cause these rare lesions. Alternatively, a sequestered fragment could cause localized hemorrhage in the epidural space and this could fall off causing the cysts described.

P128.

Microsurgery for Lumbar Disc Disease in an Ambulatory Care Setting; an Analysis of 185 Consecutive Cases

B.G. BENOIT, H.J. LESIUK, N.A. RUSSELL, P.A. SHEAR and E.A. SCHNEIDER (Ottawa, Ontario; Riyadh, Saudi Arabia; Bridgeport, U.S.A.; Sudbury, Ontario)

Our experience with microdiscectomy now comprises over 500 cases. Excellent patient acceptance led to a progressively shorter length of stay, such that the majority of our procedures for herniated lumbar disc are now done on an overnight stay basis.

A total of 185 consecutive cases treated through our Surgical Day Care Service are analyzed, and compared to an equal number of cases treated on an in-patient basis. An out-patient CT scan and/or myelogram is done, the patient is admitted on the day of surgery, maintained in the recovery room after operation, and discharged by 08:00 hours the following day. Of the 185 out-patient procedures, $12 \%$ were WCB cases and $7.5 \%$ were reoperations. Only 1 patient required admission for an adverse drug reaction. The out-patients voided sooner, were mobilized faster, and patient acceptance was very high.

Conclusion: Out-patient microdiscectomy is safe, well tolerated, and very cost effective when compared to other short stay lumbar disc procedures; i.e., automated percutaneous discectomy and chemonucleolysis.

P129.

Consequences of Ionic Manipulations on Inducing Ultrastructural Pathology in Normal Spinal Cord Axons

\section{D.L. ANTHES, E. THERIAULT and C.H. TATOR (Toronto, Ontario)}

Considerable evidence has been accumulating over the past several years to implicate calcium in mediating secondary injury events following ischemic or traumatic insults to the CNS. 
Recent in vitro studies suggest that the $\mathrm{Na} \pm / \mathrm{CA}^{2+}$ exchanger may mediate $\mathrm{CA}^{2+}$ entry into axons during $\mathrm{CNS}$ anoxia. Elevations in intracellular calcium can induce ultrastructural degeneration by a variety of mechanisms. The current study investigates ultrastructural pathology induced in normal uninjured spinal cords by ionic manipulations. Thirty-six adult female Wistar rats under halothane anesthesia, received a cervical laminectomy (C7-T2) followed by removal of the dorsal and lateral dura and arachnoid mater. The exposed spinal cord was then irrigated with $400 \mu \mathrm{l}$ of artificial CSF either alone (control) or containing one of: $20 \mu \mathrm{M}$ calcium ionophore (A23187), 500 $\mu \mathrm{M}$ benzamil (a Na+$/ \mathrm{CA}^{2+}$ exchange inhibitor), amiloride, or artificial CSF with $10 \times$ the normal $\left[\mathrm{CA}^{2+}\right]$ or $10 \times$ the normal $\left[\mathrm{Mg}^{2+}\right]$; drugs were administered in a blinded manner. Cord temperature was maintained at $37^{\circ} \mathrm{C}$ and cord immersion with CSF was verified for 30 minutes following exposure after with the surgical site was closed. Twenty-four hours later, animals were transcardially perfused with fixative and prepared for light and electron microscopic analysis. The calcium ionophore group displayed severe axonal pathology primarily in the lateral funiculus. Interestingly, the group receiving elevated extracellular $\mathrm{Mg}^{2+}$ also displayed marked pathology; especially prominent were axons containing large accumulations of organelles. Voltage-gated $\mathrm{Na}^{+}$channels from squid axons have been estimated to have permeability for $\mathrm{Mg}^{2+}$ as high as $10 \%$ of that for $\mathrm{Na}^{+}$. Hence, an increased $\mathrm{Mg}^{2+}$ concentration gradient (10×) across the axon membrane would likely lead to an increased intracellular $\left[\mathrm{Mg}^{2+}\right]$. Magnesium has been demonstrated to enhance microtubular disassembly at concentrations as low as $4 \times$ normal physiological levels and since microtubules play a key role in organelle transport, this may contribute to the observation of enhanced organelle accumulation in the $\mathrm{Mg}^{2+}$-treated animals.

\section{P130.}

An Ultrastructural Assessment of $\mathrm{Na}^{+} / \mathrm{Ca}^{2+}$ Exchange Blockade Following Experimental Spinal Cord Compression Injury

\section{D.L. ANTHES, E. THERIAULT and C.H. TATOR (Toronto, Ontario)}

Following spinal cord trauma, the consequences of white matter pathology (as opposed to grey matter) are primarily responsible for mediating the clinical sequelae of paralysis and anesthesia. The pathology observed after injury to the spinal cord is progressive involving secondary injury mechanisms such as: 1) ischemia due to vascular disruption and 2) elevations in intracellular calcium. Recently, inhibition of the $\mathrm{Na}^{+} / \mathrm{Ca}^{2+}$ exchanger has been demonstrated to protect $\mathrm{CNS}$ axons exposed to anoxia in vitro, presumably by diminished $\mathrm{Ca}^{2+}$ influx. The present study addressed the effects of $\mathrm{Na}^{+} / \mathrm{Ca}^{2+}$ exchange inhibition on axonal ultrastructural pathology following spinal cord injury. Twenty-four adult female Wistar rats were anesthetized with halothane and received a $53 \mathrm{~g}$ clip compression injury at the C8-T1 level. At 3 minutes post-injury, the dura was removed dorsally and laterally and the cord bathed with artificial CSF with or without $500 \mu \mathrm{M}$ benazmil (a Na+$/ \mathrm{Ca}^{2+}$ exchange inhibitor) delivered in a blinded fashion. Six animals from each group (benzamil or control) were sacrificed at 2 hours and 24 hours post-injury by transcardial perfusion. Spinal cords were processed for electron microscopy where photographic montages were compiled of the dorsolateral funiculus in cross- section, $400 \mu \mathrm{m}$ rostral to the injury site in the "ischemic penumbra". In a rectangle spanning $50 \mu \mathrm{m}$ in depth by $185 \mu \mathrm{m}$ in length immediately lateral to the dorsal horn, axonal ultrastructural pathology was quantitated for the appearance of specific characteristic pathological features (periaxonal swelling, organelle accumulation, vesicular myelin, axonal rupture, myelin separation and invagination). Statistical analysis indicates that benzamil may in fact be detrimental to axonal survival at 24 hours post-injury $(\mathrm{p}=0.015)$ with no effect at 2 hours post-injury. Normally, the $\mathrm{Na}^{+} / \mathrm{Ca}^{2+}$ exchanger is thought to function to assist in $\mathrm{CA}^{2+}$ efflux however, during anoxia/ischemia the exchanger is believed to operate in reverse, pumping $\mathrm{CA}^{2+}$ into the axons secondary to elevations in intraaxonal sodium. The results of this study may suggest a need for selective blockade of the exchanger in the reverse mode of operation, since nonselective exchange inhibition will also block $\mathrm{Ca}^{2+}$ efflux by exchangers operating in the forward mode and hence may increase intracellular $\left[\mathrm{Ca}^{2+}\right]$ On the fringe of the injury where anoxia is less severe, a subpopulation of exchangers is likely working in the forward direction to reduce intracellular $\left[\mathrm{Ca}^{2+}\right]$ and blockade of these forward exchangers may result in enhanced intracellular $\left[\mathrm{Ca}^{2+}\right]$ and more pronounced ultrastructural pathology in the penumbra.

\section{P131.}

The Role of 4-AP Sensitive $\mathrm{K}^{+}$Channels in the Pathogenesis of Axonal Dysfunction after Acute Spinal Cord Injury

\section{M.G. FEHLINGS, R. SETH and D. ANTHES (Toronto, Ontario)}

The mechanisms underlying conduction failure of acutely injured spinal cord axons are not clearly understood. We examined the hypothesis that axonal dysfunction secondary to activation of voltage-gated 4-aminopyridine (4-AP) sensitive $\mathrm{K}^{+}$ channels contributes to post-traumatic axonal conduction failure after acute spinal cord injury (SCI). Recordings were made from the spinal cord of rats in vivo $(\mathrm{n}=8)$ and in vitro $(\mathrm{n}=10)$. After acute $\mathrm{SCI}$ in vivo $(30 \mathrm{~g} \times 1 \mathrm{~min}$ clip) there was a $45.8 \pm 12.2$ (SD) \% decrease in the somatosensory evoked potential (SSEP) and a $84.75 \pm 5.05 \%$ decrease in the amplitude of the motor evoked potential (MEP). Topical application of $5 \mathrm{mM} 4$-AP significantly $(p<0.04)$ enhanced the amplitude of the MEP when compared with control cords superfused with artificial CSF. There was a trend toward improvement in the SSEP but this change was insignificant ( $p>0.05$ ). Application of 4-AP to the normal cord did not affect MEPs or SSEPs. In vitro studies were performed on isolated dorsal column strips. After clip injury, superfusion of the preparation with $1 \mathrm{mM} 4$-AP resulted in a significan increase in the amplitude of evoked field potentials. Ultrastructural studies showed evidence of myelin disruption, periaxonal swelling, vesicular myelin, lamellar separation and myelin invagination as early as 15 minutes after SCI. We con- 
cluded that acute SCI results in early post-traumatic demyelination which contributes to axonal dysfunction by exposure of sybmyelinic 4-AP sensitive $\mathrm{K}^{+}$channels.

\section{P132.}

Effects of Induced Hypothermia on SEPs in Patients with Chronic Spinal Cord Injury

\author{
P.J. POTTER, K.C. HAYES, J.T.C. HSIEH, G.A. DELANEY \\ and A.R. BLIGHT (London, Ontario; Chapel Hill, U.S.A.)
}

The effects of mild whole body hypothermia on sensory central conduction time and somatosensory evoked potentials (SEPs) have been investigated in control subjects $(\mathrm{n}=8)$ and patients $(n=14)$ with chronic spinal cord injury $(\mathrm{SCI})$. Mild hypothermia was induced by controlled circulation of a coolant (propylene glycol) through a "microclimate" head and vest garment (LSSI Mark VII) while reductions in oral $\left(\sim 1 .^{\circ} \mathrm{C}\right)$ and limb $\left(\sim 3.5^{\circ} \mathrm{C}\right)$ temperatures were monitored. Cooling induced a delayed onset $(\Delta 1.4 \mathrm{~ms})$ and marginally reduced amplitude of tibial nerve SEPs in control subjects, largely attributable to slowing of peripheral nerve conduction velocity $\left(\Delta=3.7 \mathrm{~ms}^{-1}\right.$. All SCI patients with recordable SEPs $(n=10)$ showed similarly delayed onset of the cortical response. In contrast to the controls, eight of the ten SCI patients showed increased amplitude of cortical SEPs. In three of the patients the increase in amplitude exceeded $100 \%$ of the pre-cooling values. The coolinginduced changes in SEP amplitude and latency reversed on rewarming although they did not all return to pre-cooling values by the time normal oral temperatures had been restored. The cooling-induced increases in cortical SEP amplitude support the a priori hypothesis that cooling would enhance central conduction in SCI patients with contusion or compressive lesions and conduction deficits due to demyelination.

\section{P133.}

\section{Gadolinium-MRI in Acute Transverse Myelitis}

D.R. MCLEAN, Z. ALKAWI, R. COATES, J. BRISMAR, S. BOHLEGA, S. OMER and B. RAHM (Riyadh, Saudi Arabia)

Acute transverse myelitis (ATM) is an acute inflammatory or demyelinating condition, generally of unknown etiology, which involves several segments of the spinal cord. A spinal cord syndrome, with motor and sensory levels developing over several hours to three weeks, is produced. The anterior spinal cord seems more vulnerable. Cerebrospinal fluid analysis shows slightly elevated protein and a moderate pleocytosis but normal glucose.

Magenetic resonance imaging (MRI) descriptions of ATM are rare especially studies using gadolinium enhancement.

We report three patients with ATM who were studied with T1, T2 and gadolinium enhanced T1 MRI. All showed similar abnormalities. The spinal cord was enlarged over several segments. The site of maximum clinical involvement correlated with a focal area of gadolinium enhancement extending over one or two spinal levels. The $\mathrm{T} 2$ images showed high signal intensity which surprisingly extended over more spinal segments than expected clinically.

This MRI picture is quite different from the changes observed in multiple sclerosis.

P134.

Three-Dimensional Analysis of the Vascular System in the Normal and Injured Rat Spinal Cord with Scanning Electron Microscopy of Vascular Corrosion Casts

\section{KOYANAGI and C.H. TATOR (Toronto, Ontario)}

The purpose of this study was to investigate vascular mechanisms in the pathophysiology of acute spinal cord injury. Female Wistar rats were transcardially perfused with polyester resin. Following digestion with sodium hypochlorite, vascular corrosion casts of the spinal cord were inspected by scanning electron microscopy. Thirteen rats were normal controls, while 19 rats were subjected to a $51 \mathrm{~g}$ acute clip compression injury at C8-T1 and then perfused at 15 minutes, 4 or 24 hours after injury. This technique clearly demonstrated the three-dimensional structure of both the arterial and venous systems in the rat spinal cord. Arteries were easily identified by the ovoid shaped impressions of endothelial nuclei. In the injured cord segment, the intramedullary capillary circulation showed almost complete avascularity although the anterior and posterior spinal arteries and their accompanying veins were all patent. Disruption, occlusion and constriction of sulcal arteries and their branches were commonly seen and accounted for the marked ischemia in the territory of the sulcal arteries. The 4 and 24 hour groups had avascular areas extending longitudinally in the posterior columns probably due to hemorrhage and occlusion of intramedullary veins. This study shows the anatomical substrate for the role of both the arteries and veins in the pathogenesis of post-traumatic ischemia.

P135.

Evaluating Post-Operative Fibrosis in a Rat Model

C. LAURYSSEN, J. KRCEK AND B. TRANMER (Calgary, Alberta)

Introduction: Epidural scarring, a manifestation of the normal process of wound repair, occurs after all spinal surgical interventions, contributing in part to the "Failed Back Syndrome". The purpose of this study is to evaluate a rat model for epidural fibrosis and test various substances with regard to efficacy in preventing post-operative epidural scarring.

Method: Adult male Wistar rats $(n \approx 100)$ of standardized age and weight were used. Under the general anesthesia, using aseptic technique, a two level laminectomy was performed, leaving an intervening laminar segment. Gelfoam, fat, sodium hyalurinate (HA) and Tisseel were applied to one level only. The other segment served at the control, and the wound was closed. Rats were sacrificed at 1,3 and 6 months. Following 
appropriate sectioning and staining, microscopic evaluation of the histology was performed.

Results: At 1, 3 and 6 months, fat provided a barrier between dura and scar. Gelfoam increased the epidural fibrosis significantly, HA was inconclusive and the Tisseel results will be presented.

Conclusion: A reproducible rat model for epidural fibrosis has been developed and the affect of the above materials on epidural fibrosis will be presented.

\section{P136.}

\section{Epidural Abscess Associated with Epidural Catheterization}

\section{MENDEZ and R.F. DEL MAESTRO (London, Ontario)}

Epidural abscess is a rare condition with an incidence of 0.2 to 1.2 per 10,000 hospital admissions. The majority of reported cases are associated with trauma, surgical procedures, intravenous drug use or hematogenous spread of infection from elsewhere in the body. We report two cases of epidural abscess associated with epidural catheter placement for analgesia. The first case presented with back pain, fever and meningeal irritation and no neurological deficits and was treated conservatively with antibiotics, removal of the epidural catheter and magnetic resonance follow-up. Complete resolution of the abscess was achieved. The second case presented with neurological deficits and was treated with a combination of antibiotics and surgical decompression with good results. The possible mechanism of abscess formation, diagnosis and treatment of patients with epidural catheters is discussed. Although aggressive surgical treatment remains the mainstay therapy in epidural abscess, non-operative management has a role in the treatment of epidural abscess caused by epidural catheter placement.

\section{P137.}

\section{A Survey on Spina Bifida in the Province of Newfoundland and Labrador}

\section{F.B. MAROUN, H. LAISHRAM, D. PRICE, R. KENNEDY and J.C. JACOB (St. John's, Newfoundland)}

A retrospective chart review of 274 patients born between 1967 and 1990 was studied. Analysis of the statistical variables revealed that the incidence per 1,000 live births had remained stationary in the Province of Newfoundland and Labrador. There was a slight female predominance $-64 \%$ were born to young mothers and the peak incidence occurred in March, April and May. A total of 179 patients out of 254 who underwent surgery were alive and underwent rehabilitation programs. Only $35 \%$ are wheelchair bound, the rest were ambulatory. One hundred and sixty-one are of school age or older, 78 are in a regular high school, 36 in special education, 6 graduated from high school and 4 are in university. Fifteen had no neurological deficit, 7 were lost to follow-up, 5 died and 9 gross mental retardation. To obtain these results these patients had multiple admissions and surgical procedures and they were followed twice a year at the rehabilitation unit. From this study the utilization of medical services and impact on the community was great. However, the majority of these patients appear to obtain a surprisingly high quality of life.

P138.

\section{Lumbar Discectomy in Children}

F.B. MAROUN, M. MIKHAIL, J.C. JACOB and A. BADEJO (St. John's, Newfoundland)

A retrospective study of 167 patients admitted with low back pain below the age of 17 were studied from 1971 to 1992. Twenty patients had lumbar disc surgery, the youngest was 9 years old. Sex distribution was equal. A significant history of trauma was found in 9 cases. Conservative management was carried for an average of 6 months duration. Six patients had neurological deficit mainly motor weakness and 1 patient with bladder symptoms. Spondylolisthesis was associated in 2 cases. Degenerative disc material was found only in 4 cases. Investigation and outcome will be discussed.

\section{P139.}

Syringomyelia Secondary to Impeded CSF Flow in the Thoracic Spinal Subarachnoid Space Caused by an Idiopathic Arachnoid Membrane

\section{M.S. LI, R. POKRUPA and J. ESPINOSA (Montreal, Quebec)}

Syringomyelia is known to be associated with Chiari I malformations, spinal tumours, spinal trauma, and arachnoiditis. Clinical presentation and modern imaging techniques usually identify the cause of a central cavity pre-operatively. We present a case of thoracic syrinx of unknown etiology pre-operatively. The patient was a healthy young male who presented with atypical left upper extremity sensory symptoms after minor neck strain. Investigations showed a high thoracic syrinx. Surgical exploration revealed a vascularized arachnoidal membrane adherent to the dorsal thoracic spinal cord. This membrane prevented rostral flow of CSF in the arachnoid space. Instead, the caudal arachnoid space communicated with a syrinx at T3. Both the cystic compartment formed by the membrane and the syrinx expanded together with respiration. Sectioning the membrane allowed free flow of CSF in the arachnoid space and collapse of the syrinx. Post-operatively the patient's symptoms improved. In presenting this unusual case we suggest a spontaneously developing arachnoid adherence as a new etiology for syringomyelia. 
P140.

\section{Posterior Subaxial Spinal Cord Compression in Rheumatoid} Arthritis

\section{D.J. FAIRHOLM (Vancouver, British Columbia)}

Rheumatoid arthritis causes laxity of the ligamentous and joint structures of the cervical spine. This results in instability and subluxations at multiple levels. Subaxial subluxations are common complications of rheumatoid arthritis and result in spinal cord compression. The common mechanical and pathological entity is a progressing subluxation at one or more levels. These are often reducible and the management consists of a posterior fusion, either with wire or lateral mass plate fixation.

A less common variant occurs when the chronic subluxation gives rise to a deformity in the posterior lamina, resulting in an increasing static posterior compression of the cord. Management of this variant requires a limited posterior laminectomy prior to a fusion procedure.

Six patients with subaxial subluxation secondary to rheumatoid arthritis have recently been evaluated and managed. Because the primary pathological and mechanical changes gave rise to a posterior laminar compression, a laminectomy was required as a primary management choice and was combined with a fusion in all cases.

The clinical, radiological and management data on this interesting sub-group of patients will be presented.

P141.

Unilateral Segmental Thoracic Hyperhidrosis with Paralysis of the Colon

S.S.C. CHOUINARD, P. DUQUETTE, G. BLAISE and J. BAILLARGEON (Montreal, Quebec)

Objective: We report the simultaneous occurrence of localized thoracic hyperhidrosis, autonomic colonic dysfunction, and loss of all sexual functions in a patient with a thoracic cancer.

Background: Rare cancer patients with localized thoracic hyperhidrosis, or gastro-intestinal motor dysfunction, attributable to sympathetic involvement have been reported. No patient has been reported with both types of manifestations.

Results: A 65-year-old man presented with acute simultaneous onset of pain and continuous profuse sweating involving dermatomes $\mathrm{T} 5$ to $\mathrm{T} 10$, and with a severe constipation and a total loss of sexual functions. Investigation revealed a thoracic cancer adjacent to the $\mathrm{T} 7$ vertebra and a paralysis of the ascending colon. A para-vertebral block reduced most symptoms.

Conclusions: In this unique case, the localisation of the tumor explained the sympathetic stimultation responsible for the hyperhydrosis and colonic hypomotility. We have no explanation for the global loss of sexual function.
P142.

Atypical Cobalamin Deficiency Presenting as Pure Motor Spastic Paraparesis

\section{A. PRASAD, K. GREWAL and T. CURRAN (St. John's, Newfoundland)}

Recent emphasis has been directed at identifying "atypical" cobalamin (Cbl) deficiencies with better screening tests. Additionally there are an increasing number of reports with "atypical" deficiency and subtle manifestations. We report a patient presenting with chronic progressive pure motor spastic paraparesis and "atypical" Cbl deficiency.

A 75-year-old woman presented with a two year history of gait difficulties and falls leading to wheelchair ambulation. Examination revealed a spastic paraparesis with brisk reflexes, ankle clonus and upgoing plantars and no objective snsory loss. The red cell indices ( $\mathrm{Hb}, \mathrm{MCV}$ ) were normal but a low $\mathrm{Cbl}$ level and a blood smear with many hypersegmented neutrophils was found. A Schilling test, CSF, EMG, NCS, SSEPs and a myelogram were normal. Parenteral $\mathrm{Cbl}$ and a 8 month followup has shown significant motor improvement.

This patient likely has "food-Cbl malabsorption". Additional confirmation of this atypical deficiency would need more sophisticated testing inaccessible to us at present.

Predominant lateral column dysfunction in Cbl deficiency is extremely rare. We are not aware of a previous report associated with an "atypical" hematological profile. The differential diagnosis for pure motor spastic paraparesis should include multiple sclerosis, primary lateral sclerosis and various metabolic causes. The case serves to highlight the need to consider atypical $\mathrm{Cbl}$ deficiency in all patients with a progressive myelopathy of unknown etiology.

\section{II - 5. CEREBROVASCULAR}

P143.

\section{Cost of Stroke}

L.T. SMURAWSKA, C.F. BLADIN, A.V. ALEXANDROV and J.W. NORRIS (Toronto, Ontario)

Stroke is the third commonest cause of death and the commonest cause of chronic neurological disability and represents a huge burden of health care cost. We decided to estimate all direct costs of stroke patients during their hospital admission to investigate the possibility of cost containment.

We evaluated the cost retrospectively in 50 randomly selected acute patients. These were 22 males (age $74 \pm 10$ ) and 28 females $(80 \pm 6)$. The mean length of stay in males was $20 \pm 22$ days while in females it was $38 \pm 48$ days $(p=0.1$ ). In survivors most males went home or to rehabilitation centres, whereas most females went home or to a nursing home. Average daily cost of stroke care was approximately $\$ 800$ per day, $\$ 15,200$ per 
male admission and $\$ 29,600$ per female admission. Therefore, in our stroke unit the total cost of the initial hospital admission is $\$ 6.2$ million for 300 patients per year.

We believe the reorganization of existing hospital services could accelerate discharge from the hospital and improve costs.

P144.

Epidemiology of Cerebrovascular Disease Among ChineseCanadians - A Retrospective Study

\section{J.Y. CHU (Etobicoke, Ontario)}

Orientals are commonly known to have more intracranial vascular stenosis and a lower incidence of carotid stenosis than Caucasians (Brust, 1975). The following is a retrospective analysis of 28 Chinese-Canadians seen in Toronto between 1990 and 1992. Their mean age was 66 (range $27-81$ ) years and average duration of symptoms 2.09 years. There were 3 severe, 13 moderate, 11 mild cases and 1 death. 18/28 had left hemispheric and $7 / 28$ right hemispheric involvement; 3 were bilateral. $71.4 \%$ $(20 / 28)$ had cerebral infarctions, of which $40 \%(8 / 20)$ were cortical and $60 \%(12 / 20)$ subcortical, due to lacunes. $10.7 \%(3 / 28)$ had intracerebral hemorrhages. Hypertension was present in $78.5 \%(22 / 28)$, while only $3.6 \%$ (1/28) had significant carotid stenosis. Nonstenosing plaques were found in $39 \%$ (11/28), diabetes mellitus in $17.9 \%$, hyperlipidemia in $21.4 \%$, smoking in $21.4 \%$, coronary heart disease in $14.3 \%$ and peripherovascular disease in $10.7 \%$. It can be concluded that the types of cerebrovascular disease found in Chinese-Canadians are not significantly different than those found in Caucasians (Kurtzke, 1985). However, the incidence of significant carotid stenosis in these patients is low, although their risk factors for cerebrovascular disease do not differ significantly from those of Caucasians.

\section{P145.}

\section{How Common are Seizures After Stroke?}

\section{C.F. BLADIN, P.J. JOHNSTON, L.T. SMURAWSKA, A.V. ALEXANDROV and J.W. NORRIS (Toronto, Ontario)}

We are currently studying the frequency, pathogensis and outcome of seizures after stroke. We evaluated 594 consecutive stroke patients of whom 568 had no prior history of epilepsy. $47 / 568$ patients $(8.3 \%)$ had seizures, including $6 / 59(10.2 \%)$ haemorrhages, and $41 / 509(8.1 \%)$ infarcts $(p=n . s)$; the majority (53\%) occurred in the first week, but a further 34\% occurred 6 to 12 months after the stroke.

Cortical lesions produced more seizures than subcortical $(\mathrm{p}<$ 0.004). 29/47 (62\%) seizures were focal motor (simple partial), or generalized tonic clonic in onset. The mean lesion volume on CT did not differentiate seizures from non-seizures for haemorrhages but did for infarcts $\left(94 \mathrm{~cm}^{3}\right.$ vs. $\left.45 \mathrm{~cm}^{3} \mathrm{p}<0.003\right)$. Seizures were not more common in cardioembolic strokes. Seizures had a worse neurological outcome $(p<0.03)$, but not mortality. Recurrent seizures occurred in $31 \%$ patients during a mean follow-up of 80.1 days. Focal slowing was the most com- mon EEG pattern in either group but in general was of no help in predicting the onset or recurrence of seizures.

Post stroke seizures are more common than previously realized and have poorer neurological outcome.

P146.

\section{Left Carotid Dominance in Stroke}

C.F. BLADIN, L. SMURAWSKA, A.V. ALEXANDROV, C.Z. ZHU and J.W. NORRIS (Toronto, Ontario; Calgary, Alberta)

In 380 consecutive patients with carotid territory infarction, 220 were left and 160 were right sided $(p<0.03)$. Carotid stenosis occurred in $48 / 67(72 \%)$ of arteries with bruits and in $25 \%$ $(78 / 313)$ without bruits $(\mathrm{p}<0.0001)$. Carotid stenosis was more frequently on the left $(81 / 126)$ than right $(45 / 126)$ side in patients with carotid bruits $(\mathrm{p}<0.03)$, and was also more severe on the left $(\mathrm{p}<0.04)$.

In 288 patients with asymptomatic carotid bruits, there was no difference in severity between the two sides, until the patients became symptomatic $(\mathrm{p}<0.04)$. Asymptomatic patients had mean age 64 years while symptomatic patients were 74 years old $(p<0.001)$ i.e., left predominance and severity of carotid stenosis evolves with age.

This age-related development of predominantly left sided symptomatic carotid stenoses appears due to differences in the anatomical level, and hence different flow characteristics, of the right and left carotid bifurcations.

\section{P147.}

Changing Patterns of Referral of Patients with Carotid Artery Disease

\section{T.J. COYNE and M.C. WALLACE (Toronto, Ontario)}

139 consecutive patients referred to a vascular neurosurgeon from Aug. 1988 - Aug. 1992 were reviewed retrospectively. The aims were to determine if referral pattern had changed since the recent NASCET study publication, and to investigate the use of carotid imaging by referring physicians. The rate of referral doubled following publication of the NASCET report in August 1991. This increase was primarily due to a 2.8 times increase in referrals from neurologists. $34 \%$ (47 of 139) of patients were referred within 1 month of symptoms, $27 \%$ (37 of 139) from 1 to 3 months, and $39 \%$ (55 of 139) more than 3 months from symptom onset. No difference was noted in time to referral, or in the type of symptom, when comparing referrals pre- and postpublication. $69 \%$ (96 of 139) of patients were referred with a Doppler study, and 24\% (33 of 139) with a carotid angiogram (usually in combination with Doppler). When imaging was performed prior to referral, $53 \%$ (56 of 104) of studies demonstrated severe stenosis $(70-99 \%)$. A greater proportion of patients were referred with this degree of stenosis post NASCET publication, with a corresponding decline in the proportion with other degrees of stenosis. 121 carotids (71 patients) were imaged by both Doppler and angiography. The degree of stenosis coincided 
in only $65 \%$ (79 of 121). Doppler underestimated the degree of angiographic stenosis in $14 \%$ (17 of 121), and overestimated in $21 \%$ ( 25 of 121$)$

Conclusions: The NASCET study has had a marked effect on referrals to a vascular neurosurgeon, particularly by neurologists. Doppler is commonly used as the sole imaging study prior to referral, but in this series inaccurately reflected the degree of angiographic stenosis in $35 \%$ of vessels studied.

\section{P148.}

\section{Validity of Stroke Diagnosis in Saskatchewan}

L. LIU, B. REEDER, A. SHUAIB and R. MAZAGRI (Saskatoon, Saskatchewan)

The purpose of this analysis is to determine the validity of the diagnosis of stroke on computerized hospital separation records to ascertain its value for stroke surveillance. The charts of patients with a primary, secondary and tertiary discharge diagnosis of "stroke" (International Classification of Diseases, 9th revision (ICD9 430, 431, 434, 434.1), "other stroke" (ICD9 $432,433,435-438)$ and "related conditions" of hemiplegia, vertigo, coma and abnormal vision were reviewed for the fiscal year 1990-1991 at 5 urban hospitals in Saskatchewan. In total 1,481 cases were identified and their charts abstracted; cases were classified according to the criteria of the 1980 U.S. National Survey of Stroke. The results showed that the diagnoses of "stroke" were sufficiently accurate: $91.4 \%$ for primary diagnosis; $87.5 \%$ for secondary and $81.8 \%$ for tertiary diagnosis. The validity of primary diagnosis of specific type of "stroke" was also high: thrombotic 85.9\% (170/198); embolic: 92.9 (13/14); intracerebral: $95.1 \%$ (39/41) and subarachnoid haemorrhage: $93.3 \%$ (14/15). A modest proportion of acute strokes were hidden among "other stroke" and "related conditions" categories, in particular $84 \%$ of the "ill-defined stroke" (ICD9 436) were reclassified as thrombotic stroke. The diagnosis in smaller hospitals was less accurate than that in larger hospitals due to the lack of access to computerized tomography and less complete recording of health information.

\section{P149.}

\section{A Randomized Telephone Survey of Knowledge Regarding Stroke Risk Factors in an Urban Population}

\author{
V. RAMSDEN, K. KHAN, A. SHUAIB and B. REEDER
}

(Saskatoon, Saskatchewan)

A random sample of individuals was selected from the Saskatoon telephone exchange (population 186,000) and a structured telephone interview conducted. $77 \%$ (104 of 135) of those contacted agreed to participate. Sixty-one were men and 43 were women. The most commonly identified risk factors for stroke were: poor diet $40 \%$ (36 of 89 ), hypertension $36 \%$ (32 of 89 ), and stress $36 \%$ (32 of 89 ). $24 \%$ (25 of 104) reported a family history of AMI and $28 \%$ (29 of 104) reported a family history of stroke. Individuals with a family history of stroke were more likely to identify hypertension as a risk factor $56 \%$ (15 of 27) than those with no family history of stroke $27 \%$ (17 of 62), $p=$ 0.011 . Individuals with no family history of stroke were more likely to identify stress as a risk factor $44 \%$ (27 of 62) than those with a family history of stroke $19 \%$ (5 of 27), $\mathrm{p}=0.024$. $80 \%$ ( 83 of 104$)$ indicated a willingness to learn more about the risk factors and $88 \%$ ( 91 of 104$)$ indicated a willingness to make changes in their own lifestyles to reduce their risk of having an event.

This assessment of knowledge will be helpful in planning public health initiatives and professional education endeavors relating to prevention and early identification of stroke.

P150.

A Multidisciplinary Approach in the Treatment of Neurovascular Lesions: The Montreal Neurological Hospital Experience

D. TAMPIERI, R. LeBLANC, K. TERBRUGGE, A. OLIVIER and M. AUBÉ (Montreal, Quebec)

The Neurovascular Interventional Group of the Montreal Neurological Hospital was formed in July, 1991. Composed of neurosurgeons, neurologists, interventional neuroradiologists, radiotherapists and functional imaging scientists it offers a multidisciplinary and multimodality approach for the evaluation and treatment of complex neurovascular lesions. Since its formation 74 patients with brain or spinal arteriovenous malformations (AVMs), dural fistulas, aneurysms, and hypervascular tumours, including hemangioblastomas, have been referred for evaluation and treatment. Our multidisciplinary and multimodality approach is illustrated by 5 brain AVM cases: the first in the posterior speech region treated by embolization alone; the second a deep paramedian AVM treated by embolization and stereotaxically-focused radiosurgery; the third a parietal AVM treated by embolization and surgery; the fourth a cerebellar AVM treated by embolization, partial resection, and stereotaxically-focused radiosurgery; and the fifth in the motor-hand region (as documented by activation-positron emission tomography) treated by stereotaxically-focused radiosurgery alone. We feel that the multidisciplinary approach affords a full assessment and offers all treatment options to patients with complex cerebrovascular lesions.

P151.

\section{Cerebral Infarction in Young Adults}

\section{A. MACKEY and R. COTE (Montreal, Quebec)}

Identifying the causes of ischemic stroke in the young may guide medical management and clarify prognosis. We report on fifty (50) young adults aged $15-45$ ( 27 men, 23 women) with a diagnosis of ischemic stroke. Investigation included complete neurological evaluation, appropriate blood tests (immunological, hypercoagulable state), cardiac tests (EKG, echocardiography, Holter), brain imaging (CT, MR), and cerebrovascular 
imaging (arteriogram, magnetic resonance angiography). Angiography was performed in 45 patients (90\%) with an abnormality rate of $68 \%$. Echocardiography had an abnormality rate of $29 \%$. Dissection of cervicocerebral arteries was the most probable diagnosis in 14 patients $(28 \%)$, cardioemboli in 8 patients $(16 \%)$, atherosclerosis in $5(10 \%)$, and coagulopathy in 1 patient. Sixteen patients in which the etiology was uncertain had one or several predisposing factors such as smoking, migraine or oral contraceptives, however, in 4 patients no factors could be identified. During a mean follow-up of 44 months, 7 patients had a recurrent ischemic stroke, most occurred within 6 months.

In this group, arterial dissection was the most frequent presumed etiology, however, in $40 \%$ of patients the diagnosis remained either uncertain or unknown. Angiography was the most sensitive investigational procedure showing a high yield. Prognosis was relatively good in terms of functional outcome.

\section{P152.}

\section{Cerebellar Infarction: Analysis of Twenty-Two Cases}

\section{J. LONG, L. YAN and D. JAN-PIN (Beijing, China; Toronto, Ontario)}

The clinical manifestations of cerebellar infarction are often non-particular and it is difficult to make such a diagnosis clinically. The diagnosis was based on pathology previously. Most of patients were overlooked until CT and MRI were used clinically. We reported 22 cases of cerebellar infarction diagnosed by CT, MRI, angiography and autopsy.

Twenty-two cases were hospitalized during 1987 - 1991. Nineteen were males, 3 were females. The ages ranged from 24 -74 years with mean age of 54.6 years. Sixteen cases had the history of hypertension, 3 had the history of brain stroke, 3 had diabetes and 2 had coronary atherosclerotic heart disease. Most of infarction occurred in PICA territory (18/22). The most common and earliest symptoms are dizziness and/or vertigo (19/22 cases), which often occurred repeatedly and often accompanied by nausea and vomiting sometimes by tinnitus, so that many patients of cerebellar infarction were misdiagnosed as ischemic attacks of vertebro-basilar artery system (TIAs), or labyrinthine lesions. Symptoms and signs of cerebellar lesion such as unsteady gait, limbs and/or trunk ataxia, dysarthria are also main clinical manifestations in cerebellar infarction. But in many patients, who were clear there were no cerebellar symptoms and signs, thus making clinical diagnosis of cerebellar infarction difficult (9/22 in our series). If brainstem infarction is simultaneous or brainstem was compressed by swollen cerebellum, the symptoms and signs due to brainstem involved such as ocular movement abnormality, facial nerve palsy and limb weakness et al. present. The severe patient is usually in coma. Rapid deterioration of consciousness suggests brainstem acute compression and the prognosis would be poor. In our series 4 cases with coma all died.

CT scan made it possible to diagnose cerebellar infarction in vivo in part patients. But CT is not a satisfactory instrument in distinguishing this disease. In our series CT scan was performed in 16 cases. Cerebellar infarction was confirmed in 6 cases only
(38\%). MRI without bony artifacts from posterior fossa, having much higher resolution, and making the infarction visualized earlier has been the most ideal instrument in diagnosing cerebellar infarction. And we can distinguish acute or chronic infarction according to MRI. In our series MRI was performed in 17 cases. In all of them cerebellar infarction was confirmed including 10 cases with normal CT scan.

\section{P153.}

\section{Five - Year Follow-Up of Carotid Endarterectomy (CEA)}

\section{R.O. HOLNESS, K. CONNOLLY and D.S. MALLOY (Halifax, Nova Scotia)}

CEA has proven short term benefit in preventing stroke due to severe carotid stenosis; however there are few long term reports on such patients. We studied 5-year outcomes of 53 consecutive patients who had CEA from January 1987 - April 1988. Ages ranged 49 - 79 years, with an equal M:F ratio. MC cerebral events occurred in $38(70 \%), 15$ of whom $(28 \%)$ had small completed stroke. Most patients had multiple risk factors for atherosclerosis; 17 had bilateral carotid stenosis; 5 had contralateral occlusion. Surgery caused low morbidity and no mortality. A severe post-operative stroke occurred in a man with unstable (Sundt grade IV) stroke in evolution. Of the remaining 52 patients 4 were lost to follow-up.

During a 5 -year period there were 14 deaths $(29 \%)$ in the other 48 patients. Two (4\%) had fatal strokes due to re-stenosis; $8(16 \%)$ died from advanced vascular disease (6 MIs); 1 from a ICH and 3 from unrelated causes. Of the 34 survivors none experienced significant strokes but 2 had CEA on the opposite side which became symptomatic. Four others had CEA on the asymptomatic contralateral stenosis and several had peripheral vascular procedures. Apart from two severely demented elderly patients, the others enjoyed good quality of life.

CEA prevents stroke but re-stenosis does occur. For those patients at high risk for re-stenosis (particularly with contralateral occlusion) careful radiological follow-up seems indicated. We will discuss the relevant literature including the role of patch grafting. It is hoped that the long term results of the NASCET and European studies will clarify the matter of re-stenosis and recurrent stroke in patients having CEA.

\section{P154.}

Transcranial Doppler and Compressed Spectral Array Monitoring during Carotid Endarterectomy

\section{T.E. HOGAN, K.P. BROWNE, R. GRIEBEL and A. SHUAIB (Saskatoon, Saskatchewan)}

Transcranial Doppler (TCD) is increasingly being used to assess the hemodynamic effects upon the middle cerebral artery circulation during carotid endarterectomy surgery. A decrease in blood flow velocities may help identify patients in whom inadequate collateral flow and impaired autoregulation results in insufficient hemispheric perfusion. This situation may require 
the replacement of a temporary intraluminal shunt to reverse conditions producing ischemia during crossclamping. The shunt though further introduces a complicating factor of emboli.

To help us better identify patients at risk for ischemia and possible shunting we chose to use a combination of TCD and Compressed Spectral Array (CSA). CSA was used to determine whether significant decreases ( $30-40 \%$ of baseline values) in mean flow velocities following clamping reflected insufficient cerebral blood flow for ischemia to occur.

We present our initial 13 patients ( 11 male, 2 female: mean age 63.8 years, range $35-81$ ) who underwent elective surgery for symptomatic stenosis. Our results showed in those patients where significant decreases to blood flow velocities occurred no CSA changes were noted. Shunts were therefore not used based on CSA. All patients did well following surgery.

\section{P155.}

A Clinical, Neuroradiologic, Neuropsychological Analysis of Unilateral Putaminal and Cortical Laminar Necrosis Following a Large Intravenous Injection of Heroin and Subsequent Survival

\section{M.S. JOG and P. MARCHETTI (Toronto, Ontario)}

Peripheral nervous system complications from heroin use have been reported. The literature on central nervous system complications of acute, high doses of intravenous heroin, is only anecdotal. Most cases cause death and only two pathological patterns are reported, namely diffuse ischemic changes, or none at all.

A case of excessively high heroin ingestion $(350 \mathrm{mg})$ with subsequent survival, is presented. The progressively unfolding clinical picture is carefully followed through its development of initial transient focal paralysis, partial motor seizures, subsequent ipsilateral dystonic posturing and persistent choreiform movements. A videotape of these is presented.

Data from immediate and follow-up computerized tomography magnetic resonance imaging, single photon emission computer tomography of the brain and neuropsychological testing is discussed in detail.

Using the latest understanding of the neuroanatomic and neurochemical structure of the basal ganglia, the pathogenetic mechanisms of the various clinical, psychiatric and radiologic features of this case, are reviewed. This rare and unique case gives a window into the protean manifestations of disorders of the basal ganglia and their connections.

\section{P156. Withdrawn}

\section{P157. Withdrawn}

P158.

\section{Correlation of Cerebral AVM Hemodynamics with Clinical} Presentation

S.P. LOWNIE, G.R. DUCKWILER, F. VINUELA and E. RAUPP (London, Ontario; Los Angeles, U.S.A.; Porto Alegre, Brazil)

Purpose: To compare AVM hemodynamics with clinical presentation.

Material: Twenty-seven patients undergoing transcatheter embolization of an AVM had feeder artery pressure (FAP) measurements made through the microcatheter.

Results: To average distance from catheter tip to AVM nidus was $3.1 \mathrm{~cm} \pm 1.9 \mathrm{~cm}$ (mean \pm S.D.). In small AVMs, mean FAP in patients presenting with hemorrhage was $56 \mathrm{~mm} \mathrm{Hg}$, in those with seizures $52 \mathrm{~mm} \mathrm{Hg}(p=N S)$. In medium AVMs, mean FAP in those with hemorrhage was $50 \mathrm{~mm} \mathrm{Hg}$, in those with seizures $39 \mathrm{~mm} \mathrm{Hg}(\mathrm{p}<0.05)$.

No patient presenting with seizures had a FAP greater than $63 \mathrm{~mm} \mathrm{Hg}$, and all patients above this level presented with cortical cerebral hemorrhage (as opposed to intraventricular, deep or posterior fossa bleeding).

Conclusion: There is an association between AVM hemodynamics and clinical course.

\section{P159.}

Surgical Versus Endovascular Treatment of Intracranial Aneurysms - Early Experience

\section{S.P. LOWNIE (London, Ontario)}

Introduction: Endovascular therapy of intracranial aneurysms with detachable platinum microcoils is emerging as a viable alternative to standard surgical treatment. The relative merits of one approach over the other were assessed in a preliminary fashion, based on a series of patients treated by the author using either modality.

Material: During a 6-month period, 25 aneurysms in 19 patients were treated either with surgery (19 aneurysms), endovascular therapy (5) or both (1).

Results: Excellent or good results were obtained in 10/14 surgical patients, $3 / 4$ endovascular patients and in the one patient treated with both methods. There were no deaths.

Of the 4 poor surgical results, 3 were due to Grade IV condition. The other poor result occurred in a Grade I patient who sustained perforator ischemia related to surgery.

In the endovascular group, there was no morbidity directly related to treatment. However, 3 out of 6 aneurysms were incompletely occluded, due to aneurysm neck width.

Conclusions: Endovascular therapy has advantages in patients:

- of advanced age $(>70)$ or frail condition

- of poor neurological grade

Surgical treatment is warranted in patients:

- with associated hematoma

- with large or giant aneurysms causing mass effect

- with wide-necked aneurysms. 
P160.

Severe Ischemic Left Ventricular Dysfunction and Acute Neurogenic Pulmonary Edema from Ruptured AComA Aneurysm

\section{S.E. BRIEN, G. MOHR and A. SPANIER (Montreal, Quebec)}

Cardiac abnormalities, particularly non-specific EKG changes have been well documented in patients with intracranial disease and in particular, subarachnoid hemorrhage.

We report a case of a 48-year-old operating room nurse who presented with a Grade 4 (Hunt-Hess) subarachnoid hemorrhage and severe neurogenic pulmonary edema soon followed by severe ischemic myocardial insufficiency, echo-cardiographic evidence of severe left ventricular dysfunction without a prior cardiac history. The day following her admission, her ejection fraction fell to 15 - $20 \%$ causing such ventricular dysfunction that inotropic agents were required to maintain a systolic blood pressure above $70 \mathrm{~mm} \mathrm{Hg}$. Fortunately, after clipping of her anterior communicating artery aneurysm, 72 hours post-rupture, her serial echocardiograms showed continued improvement so that at the time of discharge three weeks after admission, her ejection fraction had returned to her presumed normal level of $65 \%$.

Severe left ventricle dysfunction ("stunned myocardium") is a rare but important consideration in perioperative neuro-critical care and its management has not been discussed in the recent literature. The neuroanatomy and pathophysiology of left venticular dysfunction and neurogenic pulmonary edema in patients with subarachnoid hemorrhage will be discussed.

P161.

\section{Decision Analysis of Elective Surgery for Unruptured Cerebral Aneurysms}

\section{R. LeBLANC and K.J. WORSLEY (Montreal, Quebec)}

Background: The management of unruptured cerebral aneurysms is currently empirical. We have used decision analysis to place it on a more analytical footing.

Methods: We took $2 \%$ as the annual rate of rupture $(\mathbf{R})$ of aneurysms, $72.7 \%$ as the risk of death or disability with rupture $(\mathbf{M})$, and $6.5 \%$ as the average risk of elective surgery (S). Benefit of surgery over natural history was calculated from the equation $L\left\{\left[1-(1-R)^{L} M / 2\right]-S\right\}$ for life expectancy (L) corresponding to each quinquennial age group from age 15 to 100 years. Sensitivity analysis was performed to take into account increasing risk of elective surgery based on the size, accessibility, and presentation of the unruptured aneurysm, and the risk of angiography.

Results: A gain of at least one year of survival free of neurological sequelae is achieved with surgery for patients whose life expectancy is 19.5 years, corresponding to age 63.5 years for males and 68 years for females. This decreases to 42 and 47 years respectively for larger, less accessible aneurysms (surgical risk: $16.8 \%$ ). Greater benefit is achieved with longer life expectancy. The risk of angiography did not significantly affect the calculations.
P162.

Subarachnoid Hemorrhage of Unknown Cause; Needs to Pursue Angiographic Study

\section{A.M. SARRAZIN, J. RAYMOND and L.H. LEBRUN (Montreal, Quebec)}

Neuroscience Unit, Hospital St-Luc, Montreal, Canada. Over the past several years, numerous studies have emphasized the good prognosis of subarachnoid hemorrhage of unknown cause and some have recommended not repeating an initially negative cerebral angiography. The present study tries to show the potential danger of such an approach.

Out of 140 patients with subarachnoid hemorrhage diagnosed between December 1988 and January 1992, twelve had an angiography initially interpreted as normal, ten had a second angiography and three underwent a third. The films were reviewed by a second neuro-radiologist. The second reading resulted in a different interpretation as regards vasospasm or revealing an aneurysm in five of twelve of the first angiographies, in three of ten of the second angiographies and in one of the three of the third angiography. In one case, an aneurysm was identified on the third angiography in spite of good technique and absence of vasospasms as noted by the first reading of the previous two studies. We conclude that the main difficulty is in the interpretation of vasospasm. This difficulty in interpretation is important because the vasospasm justify to repeat angiography, therefore, we recommend review of normal studies by a second neuroradiologist and discuss as well means of obtaining optimal technique in angiography.

P163.

Surgical Approach for Anterior Communicating Artery Aneurysms

\section{M.W. BOJANOWSKI and M. PARÉ (Montreal, Quebec)}

The surgical management of cerebral aneurysms has remarkably improved with the development of microanatomy and microsurgery.

The fronto-temporal (pterional) approach has been standardized for aneurysms arising from the anterior circulation. However, there is some debate regarding the best approach for some complex anterior communicating artery aneurysms. The bifrontal inter-hemispheric approach has been viewed as an appropriate alternative.

We have retrospectively reviewed a series of 200 consecutive aneurysms from 1988 to 1992 . Of these, 65 aneurysms were located in the anterior communicating artery complex. We divided these aneurysms in those oriented anteriorly, superiorly and posteriorly.

All aneurysms were safely clipped by the pterional approach regardless of their size, shape, orientation, or the timing of the surgery following sub-arachnoid haemorrhage.

The particular surgical aspect for each group will be presented. 
P164.

\section{Serum Calcium and Subarachnoid Hemorrhage}

\author{
L.Y. FENG, R.H. XU, K. FAN, J.Y. CHEN, Y. LI, Y.P. WEI and \\ J.M. LU (Shijiazhuang City, P.R. China)
}

We have measured to total serum calcium in 20 normal volunteers and 56 consecutive patients with subarachnoid hemorrhage (SAH men 33, women 23, mean age 49,46) determined by cranial CT or lumber puncture. Of all the patients, there were 30 patients with disturbance of consciousness, 18 patients with worst prognosis ( 8 death, 10 deterioration), and 17 patients with clinical sign of vasospasm. The results show that the values of total serum calcium in the patients $(2.21 \pm 0.12 \mathrm{mmol} / 1)$ were significantly lower than those in normal volunteers $(2.51 \pm 0.22$ mmol $/ 1, \mathrm{t}=5,16, \mathrm{p}<0.001$ ), those in the patients with coma $(2.12 \pm 0.12 \mathrm{mmol} / 1)$ were significantly lower than those in those patients without coma $(2.31 \pm 0.14 \mathrm{mmol} / 1, \mathrm{t}=5,39, \mathrm{p}<$ $0.0001)$, those in the patients with worst prognosis $(2.11 \pm 0.13$ $\mathrm{mmol} / 1)$ were lower than those in the patients with good prognosis $(2.26 \pm 0.15 \mathrm{mmol} / 1, \mathrm{t}=3.41, \mathrm{p}<0.002)$, and those in the patients with clinical sign of vasospasm $(2.12 \pm 0.13 \mathrm{mmol} / 1)$ were also lower than those in the patients without that $(2.25 \pm$ $0.15 \mathrm{mmol} / 1, \mathrm{t}=3.06, \mathrm{p}<0.005$ ). In addition, the values of total serum calcium in the patients from one $(2.20 \pm 0.11$ $\mathrm{mmol} / \mathrm{l})$ to two weeks $(2.04 \pm 0.18 \mathrm{mmol} / \mathrm{l})$ after $\mathrm{SAH}$, were significantly different $(t=3.47, \mathrm{p}<0.002)$. These results indicate that total serum calcium is related to patients' conscious condition, prognosis and presence of vasospasm, and that it may give useful clue to direct treatment and assess prognosis in patients with SAH.

P165.

Etiology and Prognostic Indicators of Vertebrobasilar Dissection

J. MAHER, B.W. YOON, D.M. PELZ, J.H.W. PEXMAN, C.G. DRAKE and V.C. HACHINSKI (London, Ontario)

Background: Unlike carotid dissection vertebrobasilar dissection has received limited attention.

Methods: We identified 34 cases of vertebrobasilar dissection by reviewing angiographic films $(\mathrm{N}=33)$ and neurosurgical records $(\mathrm{N}=17)$, at University and Victoria Hospitals, Ontario, from 1985-1992.

Results: A possible pathogenesis was identified in 10 persons (4 neck manipulation, 3 arduous exercise, 3 fibromuscular dysplasia). Twenty (58.8\%) presented with subarachnoid hemorrhage, of whom $6(30 \%)$ were seriously disabled and $2(10 \%)$ died. This poor outcome was associated with being $\geq$ grade III (Hunt and Hess) at presentation, brainstem infarction and/or rebleeding. Fourteen $(41 \%)$ presented with vertebrobasilar ischemic events and 1 with unrelated hemifacial spasm, of whom $1(7 \%)$ died and $9(60 \%)$ were seriously disabled, this poor outcome being exclusively associated with brainstem infarction.
Conclusion: A possible pathogenesis for vertebrobasilar dissection was identified in $29 \%$ of cases. Among those with nonhemorrhagic dissection and brainstem stroke all had a poor outcome; those with a hemorrhagic dissection had a poor outcome if they were $\geq$ grade III at presentation, rebleed and/or had a brainstem stroke.

P166.

The Effect of the Anticonvulsant, Remacemide on Transient Focal Cerebral Ischemia

\section{XUE, Z.G. HUANG and A.M. BUCHAN (Ottawa, Ontario)}

Glutamate receptor antagonists have effects in the treatment of focal ischemia, despite the controversy that still remains on their mechanisms of action. In this study, a "weak" non-competitive NMDA receptor antagonist, remacemide hydrochloride, was tested in transient focal ischemia model in male Wistar and hypertensive rats (SHR). All animals sustained $2 \mathrm{hr}$ of transient focal cerebral ischemia by common carotid and right middle cerebral artery occlusion, followed by reperfusion for $22 \mathrm{hr}$ Regional cerebral blood flow and physiological conditions were monitored during experiment. The volume of cortical infarction was measured. Student's $t$ test was performed.

\begin{tabular}{llcc}
\hline & Group (n) & $\begin{array}{c}\text { Cortical Infarction } \\
(\mathbf{m m 3} \pm \mathbf{S E})\end{array}$ & Reduction (\%) \\
\hline \hline Wistar & Saline (20) & $153 \pm 20$ & \\
& Remacemide (20) & $115 \pm 20$ & 25 \\
SHRs & Saline (20) & $206 \pm 8$ & $25 * *$ \\
& Remacemide (20) & $155 \pm 14$ & 25 \\
\hline
\end{tabular}

$* *, \mathrm{p}<0.01$

The anticonvulsant, remacemide, achieved a 25 percent reduction in the volume of cortical infarction in both Wistar and SHRs.

P167.

Continuous vs. Interrupted Arterial Temporary Clipping: Results in a Rat Model of Focal Cerebral Ischemia

\section{B.I. TRANMER and M. OHTAKI (Sapporo, Japan)}

During cerebral aneurysm surgery "temporary clipping" of major cerebral arteries is common practice. This study was performed to investigate whether the brain is more tolerant of repetitive short episodes of cerebral ischemia (vessel occlusion) versus a single long period of ischemia in a rat model for focal cerebral ischemia.

In the repetitive ischemia group, the rats were subjected to three successive episodes of 1-hour middle cerebral artery occlusion at hourly intervals. The control rats underwent a continuous 3-hour insult of middle cerebral artery occlusion. The left middle cerebral artery was occluded by the endovascular 
suture method. Local cerebral blood flow was determined by hydrogen clearance technique. The neurological status was carefully assessed for 48 hours and the volume of infarction size was then evaluated.

There was no mortality and no significant difference in neurological outcome during 48 hours of observation between the two groups. Infarction volumes in both neocortex and the striatum were also not significantly different between the groups.

These results suggest that repeated temporary interruption of blood flow during neurovascular procedures may not be any safer than a single long occlusion of the same length of time.

P168.

Microvascular Anatomy of Striate Vessels in Dogs: Contribution to an Experimental Model of Forebrain Ischemia

\section{R. ST.-JACQUES, W. GORCZYCA, P. NOVAK and G. MOHR} (Montreal, Quebec)

Inspired by Brenowitz and Yonas (Surg. Neurol., 1990, 33: 247-252) a systematic study of the vascular supply of the anterior perforated substance was undertaken in 25 dogs using selective intravascular injections of acrylic paint and/or india ink. Due to the extreme development of the olfactory system in dogs, large ethmoidal arteries were found consistently. Perforating vessels from the anterior (ACA) and middle cerebral arteries (MCA) (called "striate vessels" by Brenowitz and Yonas) were found to supply the basal forebrain and basal ganglia as the equivalent of the medical and lateral lenticulostriate vessels in human. Most often located in the junction of the ethmoidal and the anterior cerebral arteries, we observed a perforating vessel resembling the human recurrent artery. Deep parenchymal perfusion territories were studied using slices rendered translucent after methyl salicylate impregnation. Middle cerebral artery perforators were found to supply the internal capsule and more lateral areas whereas the recurrent perforating vessel(s) seem to supply the globus pallidus, putamen and caudate nuclei, thus suggesting similarities with Heubner's recurrent arteries in humans. As in humans, there is few direct anastomosis between ACA and MCA perforating vessels, but selective india ink injection revealed that the two territories might communicate at the capillary level.

Improved knowledge on deep vascular territories in dogs will, hopefully, allow more selective ischemic models of the basal forebrain.

P169.

During Repetitive Cerebral Ischemia, Superoxide Dismutase is Neuroprotective at Lower Doses. Higher Dose may be Harmful, Especially in the Caudal Brain Regions D. TRUELOVE, A. SHUAIB and J. KALRA (Saskatoon,
Saskatchewan)

The mechanism for severe damage with repetitive ischemia is not understood. Because of prolonged reperfusion periods between the brief insults, we speculated that the severe damage may be secondary to excessive generation of oxygen free radicals. In this study we tested the efficacy of superoxide dismutase (SOD) in a model of repeated ischemia in gerbils. (SOD) or vehicle (saline) was delivered through osmotic pumps from the onset of the insult until the gerbils were sacrificed 6 days later. Two doses of SOD were used for the experiments [110 and 190 units per microL]. Damage was assessed with a $0-4$ point scoring system and statistical comparisons were done using the Mann-Whitney $U$ test. There was significant protection in the hippocampus [ $<<0.05]$, striatum $[\mathrm{p}<0.001]$, and the substantia nigra reticulata $[p<0.05]$ in the lower dose SOD treated group [110 units per microL]. In the animals treated with the higher does of SOD [190 units per microL], the extent of damage was no different than vehicle treated controls in the cortex, striatum and hippocampus. Compared to controls neuronal damage was however significantly more severe in the medial geniculate nucleus and the thalamus in the high-dose SOD-treated animals $(p<0.05)$. Our experiments show that the protective effects of SOD are seen at a dose of 110 units per microL per animal. Higher doses may either have no neuroprotective effects or may actually be harmful.

\section{P170.}

\section{Nitric Oxide Does Not Contribute to Ischemic CA1 Injury}

\section{H. LI and A.M. BUCHAN (Ottawa, Ontario)}

Glutamate, released by ischemia, may activate the production of nitric oxide (NO) by stimulating NO synthetase (NOS) via the NMDA receptor leading to delayed neuronal injury. We tested this hypothesis in rats exposed to severe forebrain ischemia. Adult male Wistar rats were subjected to $10 \mathrm{~min}$. of severe 4 VO ischemia. L-nitro-arginine was infused i.v. 0.5 hour preischemically. Saline-injected animals served as controls. The number of damaged hippocampal CAI neurons were counted and expressed as a percentage. A Mann-Whitney U test was employed.

\begin{tabular}{lc}
\hline Group (n) & Mean \% CA1 injury \pm SD \\
\hline \hline Saline (8) & $77 \pm 10$ \\
N-arginine & \\
$2 \mathrm{mg} / \mathrm{kg} \mathrm{(7)}$ & $80 \pm 7$ \\
$10 \mathrm{mg} / \mathrm{kg}(8)$ & $78 \pm 11$ \\
$20 \mathrm{mg} / \mathrm{kg}(3)$ & $54 \pm 13$ \\
$40 \mathrm{mg} / \mathrm{kg}(5)$ & $74 \pm 19$ \\
\hline
\end{tabular}

These data fail to show that NOS inhibition prevents in vivo ischemic injury and support our contention that the NMDA receptor is not critically involved in $\mathrm{CA} 1$ ischemic death since NOS inhibitor will prevent NMDA toxicity. 
P171.

\section{Hypothyroidism Protects the Brain During Transient Forebrain Ischemia}

A. SHUAIB, S. IJAZ, S. SULAKHE-HEMMINGS, J. KALRA, A. SENTHILSVLVAN, N. CROSBY and R. MAZAGRI (Saskatoon, Saskatchewan)

The mechanisms whereby brain cells die during brief episodes of cerebral ischemia are not fully understood. Hypothyroidism is known to decrease cerebral metabolism. We postulated that this slowing in cerebral metabolism after ischemia may be neuroprotective after transient cerebral ischemia. To test this hypothesis, a total of 10 gerbils had thyroidectomies performed two weeks prior to ischemia. Six gerbils served as euthyroid controls. All animals were exposed to five minutes of transient ischemia and sacrificed 7 days after the insult. Silver degeneration staining was used for histological evaluation. Hippocampal CAl $(p=0.001)$ and CA4 $(p<0.001)$ damage was significantly less in the hypothyroid animals. The exact mechanism of this protection is not fully understood but could be secondary to a decrease in the metabolic activity or a reduced generation of free radicals (as is seen with protection from ischemia in kidney and liver under hypothyroid conditions). Further studies are required in order to gain a better understanding of the protective effects of hypothyroidism on cerebral ischemia.

P172.

The Use of NMDA Receptor Blocker and GABA A Agonists in Repetitive Forebrain Ischemia in Gerbils

\section{S. IJAZ and A. SHUAIB (Saskatoon, Saskatchewan)}

Neuronal damage from repeated brief ischemic insults are cumulative and exceeds the effects of a similar duration single insult. We have previously shown that CGS-19755 (NMDA receptor blocker) and Muscimol (GABA agonist) significantly attenuated neuronal damage when used during repeated insults in gerbils. As the two agents work through separate mechanisms, this study was designed to test the effects of combination therapy on repeated ischemia in gerbils. Five animals received CGS-19755 (30 mg/kg) subcutaneously and Muscimol (20 $\mathrm{ng} / \mathrm{microL}$ ) into third ventricle (via osmotic pumps) and five animals were treated with saline. Histological evaluation 7 days after three 2 minute ischemic insults (one hour apart) showed significant protection (Mann Whitney $U$ test) in the hippocampus, striatum and thalamus in the treated group. The protective effects of combination therapy were however no better than the use of either agent alone. Although there is neuronal protection following ischemia with the use of glutamate antagonists or GABA agonists, these effects are not significantly enhanced if the two therapies are combined.

\section{McKenzie Prize Award}

\author{
Discrimination of Human Intracranial Tumours In Vivo \\ Using 1H MR Spectroscopic Imaging (1H MRSI) and \\ Feature Space for Spectral Pattern Recognition
}

M.C. PRUEL, D.L. COLLINS, J.G. VILLEMURE, A. OLIVIER, G. MOHR, R. POKRUPA, R. LeBLANC, R. ETHIER, W. FEINDEL and D.L. ARNOLD (Montreal, Quebec)

Conventional radiological techniques and pathological information from biopsies used to classify or histolgically grade brain tumours not infrequently yield ambiguous results. We used $1 \mathrm{H}$ MRSI to study in vivo the regional distribution of $1 \mathrm{H}$ MR signals from choline-containing compounds, phosphocreatine + creatine $(\mathrm{Cr}), \mathrm{N}$-acetylaspartate, alanine, lactate, and lipid in 35 untreated (except for corticosteroids) patients with subsequent histology-proven (Ringertz grading) brain tumours (glioblastoma multiforme $=9$; low grade astrocytoma $=12$; metastasis $=$ 9 ; meningioma $=5$ ) and 5 normal subjects. Tumours were classified using values for the 6 metabolites from multiple $1 \mathrm{~cm}^{3}$ voxels in the tumour center (normalized to contralateral $\mathrm{Cr}$ ) and a computer classification program as glioblastoma, low grade astrocytoma, metastasis, or meningioma according to the "nearest distance to the mean" in 6-dimensional feature space. All tumours in this training set were correctly assigned to the histologically-confirmed major tumour type. IH MRSI provides noninvasive chemical characterization of brain neoplasia not possible from CT and MRI. "Typical" metabolic profiles of various types of brain tumours can be recognized, and spectral patterns can be identified with different intracranial tumours that make it possible to differentiate these tumours by computer-based classifiers or artificial intelligence for spectral pattern recognition. 